\title{
Renewable Monomer Feedstocks via Olefin Metathesis: Fundamental Mechanistic Studies of Methyl Oleate Ethenolysis with the $1^{\text {st }}$ Generation Grubbs' Catalyst
}

\author{
Kenneth A. Burdett, Lori D. Harris, Peter Margl, Bob R. Maughon*, Tezi Mokhtar- \\ Zadeh, Peter C. Saucier, Eric P. Wasserman
}

The Dow Chemical Company, Corporate Research and Development

1776 Building, Midland, MI 48674

brmaughon@dow.com

Supporting Information Section 


\section{A: Ethylene solubility studies:}

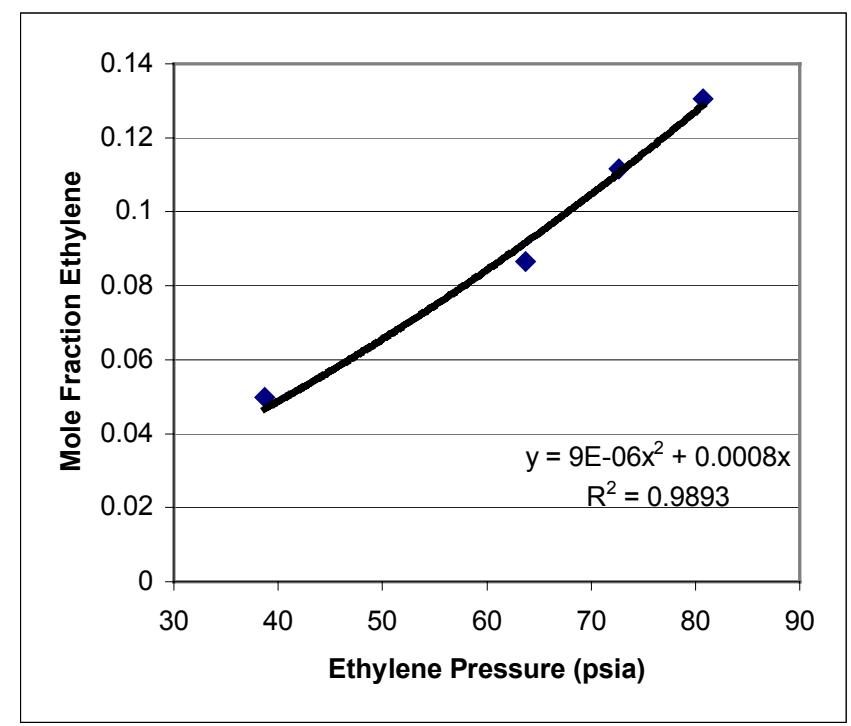

Figure S1. Ethylene solubility in methyl oleate (MO) as a function of pressure.

B: Selected lab data and kinetic modeling fit results for a range of conditions (only selected starting materials and products are shown for simplicity):

Table S1. Conversion of methyl oleate (MO) in the MO ethenolysis with catalyst $\mathbf{1}^{\mathrm{a}}$

\begin{tabular}{|c|c|c|c|c|c|c|c|c|c|}
\hline & \multicolumn{2}{|c|}{$\begin{array}{l}\text { Methyl Oleate } \\
(\mathrm{mmol})\end{array}$} & \multicolumn{2}{|c|}{$\begin{array}{l}\text { Methyl 9- } \\
\text { Decenoate } \\
(\mathrm{mmol})\end{array}$} & \multicolumn{2}{|c|}{ Diester (mmol) } & \multicolumn{3}{|c|}{$\mathrm{Ru}(\mathrm{mmol} * 1000)$} \\
\hline Time & Model & $\mathrm{Lab}$ & Model & $\mathrm{Lab}$ & Model & $\mathrm{Lab}$ & Decayed & $4 / 5$ & 6 \\
\hline 0 & 33.2 & 33.2 & & & & & 0.0 & 4.5 & 2.9 \\
\hline 8 & 29.0 & 27.8 & 4.0 & 5.2 & 0.1 & 0.2 & 0.2 & 4.0 & 3.3 \\
\hline 36 & 21.7 & 18.4 & 10.2 & 14.0 & 0.6 & 0.5 & 1.0 & 3.0 & 3.5 \\
\hline 69 & 17.4 & 15.0 & 13.6 & 16.9 & 1.1 & 0.7 & 1.9 & 2.3 & 3.2 \\
\hline 121 & 13.8 & 13.1 & 16.2 & 18.4 & 1.6 & 0.9 & 3.1 & 1.7 & 2.7 \\
\hline 187 & 11.5 & 11.9 & 17.9 & 19.7 & 1.9 & 1.1 & 4.3 & 1.1 & 2.0 \\
\hline 1071 & 8.4 & 7.8 & 20.1 & 21.6 & 2.4 & 1.8 & 7.4 & 0.0 & 0.0 \\
\hline
\end{tabular}

${ }^{\mathrm{a}}$ Conditions: $30^{\circ} \mathrm{C} ; 60$ psig ethylene; $4500: 1[\mathrm{MO}] /[1] ;[\mathrm{MO}]=2.56 \mathrm{M}$. 
Table S2. Conversion of methyl oleate (MO) in the MO ethenolysis with catalyst $\mathbf{1}^{\mathrm{a}}$

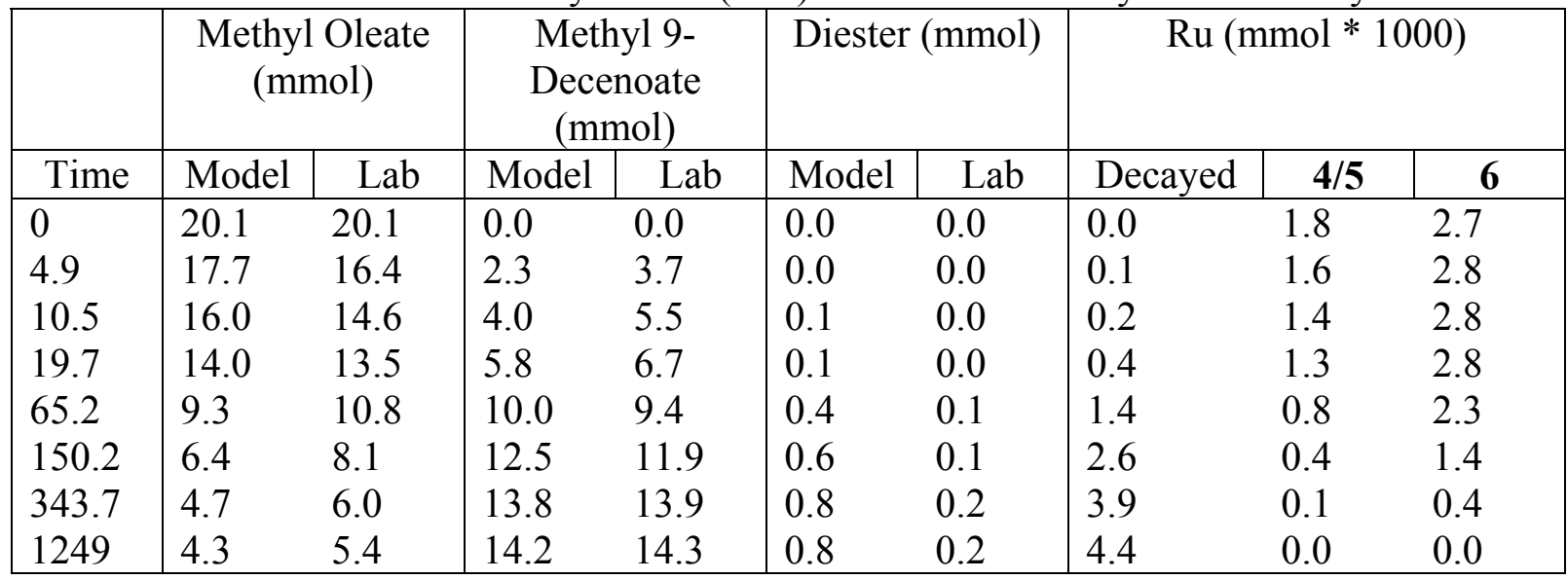

${ }^{a}$ Conditions: $30^{\circ} \mathrm{C} ; 60$ psig ethylene; $4500: 1[\mathrm{MO}] /[1]$; [MO] $=1.04 \mathrm{M}$ diluted in hexanes.

Table S3. Conversion of methyl oleate (MO) in the MO ethenolysis with catalyst 1 in the presence of products to approximate $60 \%$ conversion $^{\mathrm{a}}$

\begin{tabular}{|l|l|l|l|l|l|l|l|c|c|}
\hline & \multicolumn{2}{|c|}{$\begin{array}{c}\text { Methyl Oleate } \\
(\mathrm{mmol})\end{array}$} & \multicolumn{2}{c|}{$\begin{array}{c}\text { Methyl 9- } \\
\text { Decenoate } \\
(\mathrm{mmol})\end{array}$} & \multicolumn{2}{|c|}{ Diester (mmol) } & \multicolumn{3}{c|}{$\mathrm{Ru}(\mathrm{mmol}$ * 1000) } \\
\hline Time & Model & Lab & Model & Lab & Model & Lab & Decayed & $\mathbf{4 / 5}$ & $\mathbf{6}$ \\
\hline 0 & 13.4 & 13.4 & 20.0 & 20.0 & 0.0 & 0.0 & 0.0 & 2.6 & 4.8 \\
7 & 12.9 & 12.5 & 20.1 & 20.3 & 0.2 & 0.2 & 0.3 & 2.5 & 4.6 \\
35 & 11.5 & 11.4 & 20.5 & 20.9 & 0.7 & 0.3 & 1.2 & 2.2 & 4.0 \\
67 & 10.4 & 11.1 & 20.8 & 21.2 & 1.1 & 0.4 & 2.2 & 1.8 & 3.4 \\
119 & 9.3 & 10.7 & 21.0 & 21.6 & 1.5 & 0.5 & 3.4 & 1.3 & 2.6 \\
184 & 8.5 & 10.3 & 21.2 & 21.5 & 1.8 & 0.5 & 4.6 & 1.0 & 1.9 \\
1068 & 7.3 & 8.3 & 21.6 & 22.8 & 2.3 & 1.1 & 7.4 & 0.0 & 0.0 \\
\hline
\end{tabular}

${ }^{\mathrm{a}}$ Conditions: $30^{\circ} \mathrm{C}$; 60 psig ethylene; $[1]=0.57 \mathrm{mM}$; Starting at $60 \%$ conversion, [MO] $=0.80 \mathrm{M}$, [1-

Decene] $=1.18 \mathrm{M}$, [Methyl 9-Decenoate] $=1.21 \mathrm{M}$; [9-Octadecene] $=0.077 \mathrm{M}$.

Table S4. Conversion of methyl oleate (MO) in the MO ethenolysis with catalyst $\mathbf{1}$ in the presence of products to approximate $22 \%$ conversion $^{\mathrm{a}}$

\begin{tabular}{|l|l|l|l|l|l|l|l|c|c|}
\hline & \multicolumn{2}{|c|}{$\begin{array}{c}\text { Methyl Oleate } \\
\text { (mmol) }\end{array}$} & \multicolumn{2}{c|}{$\begin{array}{c}\text { Methyl 9- } \\
\text { Decenoate } \\
\text { (mmol) }\end{array}$} & \multicolumn{2}{c|}{ Diester (mmol) } & \multicolumn{3}{c|}{ Ru (mmol * 1000) } \\
\hline \multicolumn{1}{|c|}{ Time } & Model & Lab & Model & Lab & Model & Lab & Decayed & $\mathbf{4 / 5}$ & $\mathbf{6}$ \\
\hline 0 & 26.7 & 26.7 & 7.3 & 7.3 & 0.0 & 0.0 & 0.0 & 2.6 & 4.8 \\
14 & 23.2 & 23.9 & 10.1 & 9.9 & 0.2 & 0.2 & 0.3 & 2.5 & 4.6 \\
36 & 19.5 & 19.4 & 12.9 & 13.7 & 0.7 & 0.3 & 1.2 & 2.2 & 4.0 \\
66 & 16.4 & 16.0 & 15.2 & 16.7 & 1.1 & 0.4 & 2.2 & 1.8 & 3.4 \\
118 & 13.3 & 14.0 & 17.4 & 18.5 & 1.5 & 0.5 & 3.4 & 1.3 & 2.6 \\
183 & 11.3 & 12.9 & 18.8 & 19.2 & 1.8 & 0.5 & 4.6 & 1.0 & 1.9 \\
1067 & 8.4 & 9.2 & 20.7 & 21.5 & 2.3 & 1.1 & 7.4 & 0.0 & 0.0 \\
\hline
\end{tabular}

${ }^{\mathrm{a}}$ Conditions: $30^{\circ} \mathrm{C} ; 60 \mathrm{psig}$ ethylene; $[\mathbf{1}]=0.57 \mathrm{mM}$; Starting at $22 \%$ conversion, [MO]=1.92 M, [1-

Decene $]=0.48 \mathrm{M}$, [Methyl 9-Decenoate $]=0.52 \mathrm{M}$, [9-Octadecene] $=0.020 \mathrm{M}$. 
Table S5. Conversion of methyl oleate (MO) in the MO ethenolysis with catalyst $\mathbf{1}^{\mathrm{a}}$

\begin{tabular}{|l|c|c|c|c|c|c|c|c|c|}
\hline & \multicolumn{2}{|c|}{$\begin{array}{c}\text { Methyl Oleate } \\
(\mathrm{mmol})\end{array}$} & \multicolumn{2}{c|}{$\begin{array}{c}\text { Methyl 9- } \\
\text { Decenoate } \\
(\mathrm{mmol})\end{array}$} & \multicolumn{2}{l|}{ Diester (mmol) } & \multicolumn{3}{c|}{$\mathrm{Ru}(\mathrm{mmol}$ * 1000) } \\
\hline Time & Model & Lab & Model & Lab & Model & Lab & Decayed & $\mathbf{4 / 5}$ & $\mathbf{6}$ \\
\hline 0 & 33.4 & 33.4 & 0.0 & 0.0 & 0.0 & 0.0 & 0.0 & 2.9 & 1.8 \\
130 & 15.8 & 14.6 & 14.8 & 17.1 & 1.4 & 0.7 & 2.0 & 1.1 & 1.6 \\
234 & 12.9 & 12.7 & 16.9 & 18.6 & 1.8 & 0.9 & 3.1 & 0.6 & 1.0 \\
1096 & 10.2 & 10.0 & 18.8 & 20.2 & 2.2 & 1.3 & 4.7 & 0.0 & 0.0 \\
\hline
\end{tabular}

${ }^{a}$ Conditions: $30^{\circ} \mathrm{C} ; 60$ psig ethylene; 7000:1 [MO]/[1]; [MO] $=2.56 \mathrm{M}$.

Table S6. Conversion of methyl oleate (MO) in the MO ethenolysis with catalyst $\mathbf{1}^{\mathrm{a}}$

\begin{tabular}{|l|c|c|c|c|c|c|c|c|c|}
\hline & \multicolumn{2}{|c|}{$\begin{array}{c}\text { Methyl Oleate } \\
(\mathrm{mmol})\end{array}$} & \multicolumn{2}{c|}{$\begin{array}{c}\text { Methyl 9- } \\
\text { Decenoate } \\
(\mathrm{mmol})\end{array}$} & \multicolumn{2}{c|}{ Diester (mmol) } & \multicolumn{3}{c|}{$\mathrm{Ru}\left(\mathrm{mmol}^{*} 1000\right)$} \\
\hline Time & Model & Lab & Model & Lab & Model & Lab & Decayed & $\mathbf{4 / 5}$ & $\mathbf{6}$ \\
\hline 0 & 33.4 & 33.4 & 0.0 & 0.0 & 0.0 & 0.0 & 0.0 & 0.4 & 0.2 \\
35 & 28.9 & 26.5 & 4.3 & 6.6 & 0.1 & 0.1 & 0.1 & 0.3 & 0.2 \\
135 & 25.1 & 24.9 & 7.6 & 8.1 & 0.4 & 0.1 & 0.2 & 0.2 & 0.2 \\
239 & 23.3 & 24.7 & 9.1 & 8.2 & 0.5 & 0.1 & 0.4 & 0.1 & 0.1 \\
1105 & 20.9 & 24.8 & 11.0 & 8.2 & 0.8 & 0.1 & 0.6 & 0.0 & 0.0 \\
\hline
\end{tabular}

${ }^{\mathrm{a}}$ Conditions: $30^{\circ} \mathrm{C} ; 60$ psig ethylene; $50000: 1[\mathrm{MO}] /[1] ;[\mathrm{MO}]=2.56 \mathrm{M}$.

Table S7. Conversion of methyl oleate (MO) in the MO ethenolysis with catalyst $\mathbf{1}^{\mathrm{a}}$

\begin{tabular}{|l|c|c|c|c|c|c|c|c|c|}
\hline & \multicolumn{2}{|c|}{$\begin{array}{c}\text { Methyl Oleate } \\
\text { (mmol) }\end{array}$} & \multicolumn{2}{c|}{$\begin{array}{c}\text { Methyl 9- } \\
\text { Decenoate } \\
\text { (mmol) }\end{array}$} & \multicolumn{2}{l|}{ Diester (mmol) } & \multicolumn{3}{c|}{ Ru (mmol * 1000) } \\
\hline \multicolumn{1}{|c|}{} & Model & Lab & Model & Lab & Model & Lab & Decayed & $\mathbf{4 / 5}$ & $\mathbf{6}$ \\
\hline 0 & 33.4 & 33.4 & 0.0 & 0.0 & 0.0 & 0.0 & 0.0 & 0.2 & 0.1 \\
38.5 & 29.9 & 28.0 & 3.3 & 5.0 & 0.1 & 0.1 & 0.0 & 0.2 & 0.1 \\
115.5 & 27.6 & 27.9 & 5.4 & 5.0 & 0.2 & 0.1 & 0.1 & 0.1 & 0.1 \\
310.5 & 25.3 & 28.0 & 7.4 & 5.0 & 0.3 & 0.1 & 0.2 & 0.0 & 0.0 \\
\hline
\end{tabular}

${ }^{\mathrm{a}}$ Conditions: $30^{\circ} \mathrm{C} ; 60$ psig ethylene; $100000: 1[\mathrm{MO}] /[1] ;[\mathrm{MO}]=2.56 \mathrm{M}$. 
Table S8. Conversion of methyl oleate (MO) in the MO ethenolysis with catalyst $\mathbf{1}^{\mathrm{a}}$

\begin{tabular}{|c|c|c|c|c|c|c|c|c|c|}
\hline & \multicolumn{2}{|c|}{$\begin{array}{l}\text { Methyl Oleate } \\
(\mathrm{mmol})\end{array}$} & \multicolumn{2}{|c|}{$\begin{array}{l}\text { Methyl 9- } \\
\text { Decenoate } \\
\text { (mmol) }\end{array}$} & \multicolumn{2}{|c|}{ Diester $(\mathrm{mmol})$} & \multicolumn{3}{|c|}{$\mathrm{Ru}\left(\mathrm{mmol}^{*} 1000\right)$} \\
\hline Time & Model & $\mathrm{Lab}$ & Model & $\mathrm{Lab}$ & Model & $\mathrm{Lab}$ & Decayed & $4 / 5$ & 6 \\
\hline 0 & 33.3 & 33.3 & 0.0 & 0.0 & 0.1 & 0.1 & 0.0 & 5.9 & 1.5 \\
\hline 23 & 27.3 & 29.9 & 5.2 & 3.1 & 0.5 & 0.3 & 0.4 & 4.9 & 2.2 \\
\hline 46 & 23.6 & 25.2 & 7.8 & 7.3 & 1.0 & 0.6 & 0.8 & 4.2 & 2.4 \\
\hline 64 & 21.4 & 19.4 & 9.3 & 12.3 & 1.4 & 1.1 & 1.1 & 3.8 & 2.5 \\
\hline 122 & 17.0 & 17.1 & 12.0 & 13.5 & 2.3 & 1.6 & 2.2 & 2.9 & 2.3 \\
\hline 364 & 11.6 & 14.6 & 15.0 & 14.8 & 3.4 & 2.4 & 5.3 & 1.0 & 1.0 \\
\hline 1279 & 10.2 & 13.8 & 15.8 & 14.9 & 3.7 & 27 & 7.3 & 0.0 & 0.0 \\
\hline
\end{tabular}

${ }^{a}$ Conditions: $30^{\circ} \mathrm{C} ; 15$ psig ethylene; $4500: 1[\mathrm{MO}] /[1] ;[\mathrm{MO}]=2.56 \mathrm{M}$.

Table S9. Conversion of methyl oleate (MO) in the MO ethenolysis with catalyst $\mathbf{1}^{\mathrm{a}}$

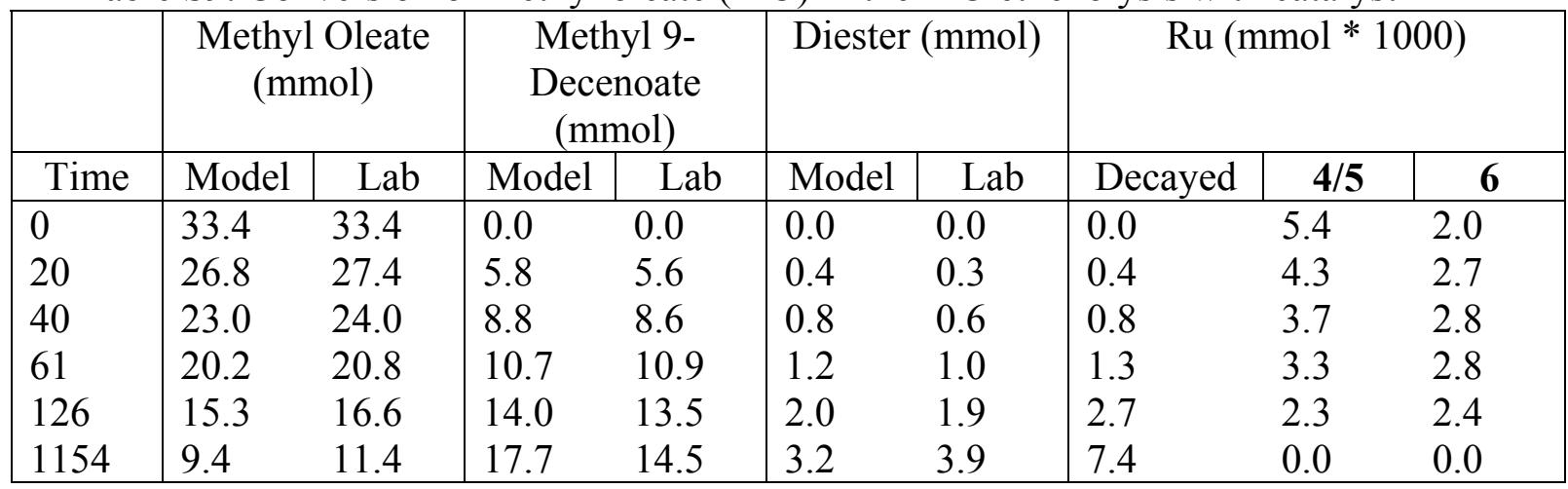

${ }^{\text {a }}$ Conditions: $30^{\circ} \mathrm{C} ; 30$ psig ethylene; $4500: 1[\mathrm{MO}] /[1] ;[\mathrm{MO}]=2.56 \mathrm{M}$.

Table S10. Coupling of Methyl 9-Decenoate with catalyst $\mathbf{1}^{\text {a }}$

\begin{tabular}{|l|ll|l|l|l|l|r|}
\hline & \multicolumn{2}{|c|}{$\begin{array}{c}\text { Methyl 9- } \\
\text { Decenoate } \\
\text { (mmol) }\end{array}$} & \multicolumn{3}{c|}{ Diester (mmol) } & \multicolumn{3}{c|}{ Ru (mmol * 1000) } \\
\hline \multicolumn{1}{|c|}{ Time } & Model & Lab & Model & Lab & Decayed & $\mathbf{4 / 5}$ & $\mathbf{6}$ \\
\hline 0 & 63.9 & 63.9 & 0.5 & 0.5 & 0.0 & 2.7 & 11.7 \\
5 & 63.0 & 62.1 & 1.0 & 1.5 & 0.5 & 2.7 & 11.3 \\
14 & 61.5 & 60.9 & 1.8 & 2.1 & 1.3 & 2.6 & 10.6 \\
20 & 60.6 & 59.9 & 2.2 & 2.5 & 1.7 & 2.5 & 10.2 \\
53 & 56.9 & 56.5 & 4.0 & 4.3 & 4.1 & 2.2 & 8.1 \\
124 & 53.0 & 55.1 & 6.0 & 4.9 & 7.8 & 1.5 & 5.1 \\
247 & 50.7 & 53.6 & 7.2 & 5.7 & 11.3 & 0.8 & 2.4 \\
\hline
\end{tabular}

${ }^{a}$ Conditions: $30^{\circ} \mathrm{C}$; 50 psig nitrogen; 4503:1 [Methyl 9-decenoate]/[1]; [Methyl 9decenoate $]=3.35 \mathrm{M}$ where the reaction was run essential neat with a small amount of added internal standard. 
Table S11. Coupling of Methyl 9-Decenoate with catalyst $\mathbf{1}^{\mathrm{a}}$

\begin{tabular}{|c|cc|c|c|c|c|c|}
\hline & \multicolumn{2}{|c|}{$\begin{array}{c}\text { Methyl 9- } \\
\text { Decenoate } \\
\text { (mmol) }\end{array}$} & \multicolumn{2}{l|}{ Diester (mmol) } & \multicolumn{3}{|c|}{ Ru (mmol * 1000) } \\
\hline Time & Model & Lab & Model & Lab & Decayed & $\mathbf{4 / 5}$ & $\mathbf{6}$ \\
\hline 0 & 64.2 & 64.2 & 0.4 & 0.4 & 0.0 & 1.8 & 7.1 \\
4 & 63.6 & 62.5 & 0.7 & 1.3 & 0.2 & 1.8 & 6.9 \\
10 & 62.8 & 61.7 & 1.1 & 1.7 & 0.5 & 1.7 & 6.6 \\
22 & 61.3 & 60.3 & 1.9 & 2.4 & 1.2 & 1.6 & 6.1 \\
48 & 58.8 & 57.2 & 3.1 & 3.9 & 2.3 & 1.5 & 5.1 \\
124 & 54.4 & 55.7 & 5.3 & 4.7 & 4.8 & 1.0 & 3.1 \\
254 & 51.5 & 54.1 & 6.7 & 5.4 & 7.0 & 0.5 & 1.4 \\
\hline
\end{tabular}

${ }^{a}$ Conditions: $30^{\circ} \mathrm{C}$; 50 psig nitrogen; 7302:1 [Methyl 9-decenoate]/[1]; [Methyl 9decenoate $]=3.35 \mathrm{M}$ where the reaction was run essential neat with a small amount of added internal standard.

Table S12. Coupling of 1-Decene with catalyst $\mathbf{1}^{\mathrm{a}}$

\begin{tabular}{|c|c|c|c|c|c|c|c|}
\hline & \multicolumn{2}{|c|}{$\begin{array}{c}\text { 1-Decene } \\
(\mathrm{mmol})\end{array}$} & \multicolumn{2}{|c|}{$\begin{array}{l}\text { 9-Octadecene } \\
(\mathrm{mmol})\end{array}$} & \multicolumn{3}{|c|}{$\mathrm{Ru}(\mathrm{mmol} * 1000)$} \\
\hline Time & Model & $\mathrm{Lab}$ & Model & $\mathrm{Lab}$ & Decayed & $4 / 5$ & 6 \\
\hline 0 & 83.5 & 83.5 & 0.6 & 0.6 & 0.0 & 3.8 & 15.0 \\
\hline 10 & 80.9 & 77.2 & 1.9 & 3.8 & 1.2 & 3.7 & 13.9 \\
\hline 20 & 78.7 & 74.8 & 3.0 & 4.9 & 2.2 & 3.6 & 12.9 \\
\hline 56 & 73.0 & 69.2 & 5.9 & 7.8 & 5.5 & 3.2 & 10.1 \\
\hline 127 & 67.2 & 65.8 & 8.7 & 9.4 & 10.1 & 2.2 & 6.4 \\
\hline 250 & 63.7 & 63.9 & 10.5 & 10.4 & 14.6 & 1.1 & 3.1 \\
\hline
\end{tabular}

${ }^{a}$ Conditions: $30^{\circ} \mathrm{C} ; 50$ psig nitrogen; $4500: 1$ [1-Decene]/[1]; [1-Decene] $=4.3 \mathrm{M}$ where the reaction was run essential neat with a small amount of added internal standard.

Table S13. Coupling of 1-Decene with catalyst $\mathbf{1}^{\mathrm{a}}$

\begin{tabular}{|c|c|c|c|c|c|c|c|}
\hline & \multicolumn{2}{|c|}{$\begin{array}{c}\text { 1-Decene } \\
(\mathrm{mmol})\end{array}$} & \multicolumn{2}{|c|}{$\begin{array}{c}\text { 9-Octadecene } \\
(\mathrm{mmol})\end{array}$} & \multicolumn{3}{|c|}{$\mathrm{Ru}\left(\mathrm{mmol}^{*} 1000\right)$} \\
\hline Time & Model & Lab & Model & Lab & Decayed & $4 / 5$ & 6 \\
\hline 0 & 84.1 & 84.1 & 0.3 & 0.3 & 0.0 & 1.8 & 7.1 \\
\hline 6 & 83.2 & 82.3 & 0.7 & 1.2 & 0.3 & 1.8 & 6.8 \\
\hline 10 & 82.6 & 81.2 & 1.0 & 1.8 & 0.5 & 1.7 & 6.6 \\
\hline 17 & 81.6 & 79.8 & 1.5 & 2.4 & 0.9 & 1.7 & 6.3 \\
\hline 39 & 79.0 & 76.9 & 2.8 & 3.9 & 1.9 & 1.5 & 5.4 \\
\hline 115 & 73.4 & 73.1 & 5.7 & 5.8 & 4.5 & 1.0 & 3.3 \\
\hline 245 & 69.4 & 71.0 & 7.7 & 6.8 & 6.9 & 0.5 & 1.5 \\
\hline
\end{tabular}

${ }^{a}$ Conditions: $30^{\circ} \mathrm{C} ; 50$ psig nitrogen; $9514: 1$ [1-Decene]/[1]; [1-Decene] $=4.3 \mathrm{M}$ where the reaction was run essential neat with a small amount of added internal standard.

C: Molecular modeling coordinates for catalyst intermediates and transition states: 
$\begin{array}{lllll}\text { C } & -0.38589999080 & 0.10670000315 & -0.25900000334\end{array}$

$\mathrm{Ru} 0.53530001640 \quad 1.6459000111 \quad 0.022399999201$

Cl $1.4876999855 \quad 2.0968000889-2.1066999435$

$\mathrm{Cl} 0.72170001268 \quad 1.71300005912 .3886001110$

C $0.39089998603-1.1754000187-0.27900001407$

C $0.27630001307-1.8934999704-1.6438000202$

C $1.0365999937-3.2230000496-1.6672999859$

$\mathrm{H}-1.4598000050 \quad 0.016300000250-0.44839999080$

H $1.4507000446-1.0010000467-0.044199999422$

$\mathrm{H}-0.0013999999501-1.8269000053 \quad 0.51920002699$

$\mathrm{H}-0.78430002928-2.0660998821-1.8701000214$

$\mathrm{H} \quad 0.66219997406-1.2258000374-2.4224998951$

$\mathrm{H} \quad 0.94429999590-3.7107000351-2.6431999207$

H $2.1033999920-3.0692000389-1.4702999592$

H $0.65289998055-3.9144001007-0.90839999914$

P $-1.15110003953 .1712000370-0.17509999871$

C $-1.95570003993 .2158000469-1.8747999668$

C $-2.36739993101 .8447999954-2.4518001080$

C $-2.8150000572 \quad 1.9854999781-3.9181001186$

C $-3.94409990313 .0132999420-4.0816001892$

C $-3.5423998833 \quad 4.3713998795 \quad-3.4886999130$

C $-3.1059000492 \quad 4.2371997833-2.0183000565$

C $-2.4223999977 \quad 3.0174999237 \quad 1.2120000124$

C $-3.2653999329 \quad 4.2767000198 \quad 1.5246000290$

C $-4.1290001869 \quad 4.0578999519 \quad 2.7815001011$

C $-5.0310997963 \quad 2.8234000206 \quad 2.6482000351$

C $-4.2024002075 \quad 1.5750000477 \quad 2.3164999485$

C $-3.3394000530 \quad 1.7850999832 \quad 1.0599999428$

C $-0.47979998589 \quad 4.9229998589 \quad 0.022700000554$

C $0.27700001001 \quad 5.1052999496 \quad 1.3583999872$

C $0.683499991896 .5749001503 \quad 1.5628000498$

C $1.5297000408 \quad 7.0921001434 \quad 0.39079999924$

C $0.80390000343 \quad 6.8850002289-0.94599997997$

C $0.38929998875 \quad 5.4165000916-1.1553000212$

H $-1.1115000248 \quad 3.5541000366-2.4897999763$

$\mathrm{H}-1.5211999416 \quad 1.1568000317-2.3975000381$

$\mathrm{H}-3.18829989431 .4138000011-1.8636000156$

$\mathrm{H}-1.9505000114 \quad 2.2941999435 \quad-4.5222997665$

$\mathrm{H}-3.13000011441 .0068000555-4.3000998497$

$\mathrm{H}-4.2097001076 \quad 3.1240000725-5.1396999359$

$\mathrm{H}-4.84569978712 .6484000683-3.5680000782$

$\mathrm{H}-2.7121000290 \quad 4.7936000824-4.0722999573$

$\mathrm{H}-4.37260007865 .0847001076-3.5615000725$

$\mathrm{H}-3.96650004393 .9052000046-1.4235999584$

$\mathrm{H}-2.8134999275 \quad 5.2195000648-1.6312999725$

H $-1.7653000355 \quad 2.8273000717 \quad 2.0710000992$

$\mathrm{H}-2.6238000393 \quad 5.1479001045 \quad 1.6849999428$

$\mathrm{H}-3.9200000763 \quad 4.5151000023 \quad 0.67750000954$

$\mathrm{H}-3.4681999683 \quad 3.9328999519 \quad 3.6505999565$

$\mathrm{H}-4.7304000854 \quad 4.9556999207 \quad 2.9700999260$

H $-5.6038999557 \quad 2.66840004923 .5703001022$

$\mathrm{H}-5.76499986652 .9953999519 \quad 1.8471000195$

$\mathrm{H}-3.54460000991 .33580005173 .1631999016$

$\mathrm{H}-4.8569002151 \quad 0.70700001717 \quad 2.1700999737$

H $-3.9993000031 \quad 1.91740000250 .19220000505$

$\mathrm{H}-2.7418999672 \quad 0.88789999485 \quad 0.88099998236$

H $-1.3769999743 \quad 5.55660009380 .054099999368$

$\mathrm{H}-0.32310000062 \quad 4.7595000267 \quad 2.2058000565$

H $1.1746000051 \quad 4.4758000374 \quad 1.3550000191$

$\mathrm{H}-0.22200000286 \quad 7.1922998428 \quad 1.6598000526$

H $1.2324999571 \quad 6.6742000580 \quad 2.5065999031$

$\mathrm{H} 1.7719000578 \quad 8.15240001680 .53210002184$

H $2.4853000641 \quad 6.5494999886 \quad 0.37020000815$

$\mathrm{H}-0.093500003219 \quad 7.5208001137-0.97329998016$

$\mathrm{H} 1.4385999441 \quad 7.2051000595-1.7810000181$

$\mathrm{H} 1.27830004694 .7834000587-1.2510999441$

$\mathrm{H}-0.15109999478 \quad 5.3326001167-2.1033000946$

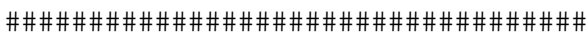

$14 \mathrm{e}$-methylidene

58

DOW RESTRICTED - For internal use only 
$\mathrm{Ru}-4.0226001740 \quad 2.4063999653-0.79949998856$

C $-3.6203999519 \quad 3.9516999722 \quad 0.051399998367$

P $-1.90509998801 .6283999681-1.1382000446$

C $-1.94649994370 .0049000000581-2.1154000759$

C $-3.2009000778-0.82950001955-1.7608000040$

C $-3.1594998837-2.2105000019-2.4368000031$

C $-3.0257999897-2.0868999958-3.9604001045$

C $-1.8107000589-1.2279000282-4.3344001770$

C $-1.8377000093 \quad 0.15240000188-3.6496999264$

C $-0.82139998674 \quad 2.7555999756 \quad-2.1923999786$

C $-0.896899998194 .2671999931-1.8956999779$

C $-0.12409999967 \quad 5.0602998734-2.9663000107$

C $1.3356000185 \quad 4.5981998444-3.0910999775$

C $1.42030000693 .0857999325-3.3431000710$

C $0.65399998426 \quad 2.2971000671 \quad-2.2660999298$

C $-0.87470000982 \quad 1.1900000572 \quad 0.37290000916$

C $-0.77009999752 \quad 2.35470008851 .3798999786$

C $0.20200000703 \quad 2.0179998875 \quad 2.5246999264$

C $-0.19769999385 \quad 0.71929997206 \quad 3.2388999462$

C $-0.32330000401-0.43909999728 \quad 2.2395999432$

C $-1.2999000549-0.106799997391 .0959999561$

$\begin{array}{lllll}\mathrm{C} 1 & -4.7501997948 & 0.98809999228 & 0.93819999695\end{array}$

C1 $-4.30060005192 .9595000744 \quad-3.0831000805$

H $-2.6473000050 \quad 4.32539987560 .37419998646$

$\mathrm{H}-4.4854001999 \quad 4.5906000137 \quad 0.28880000114$

H $-1.0579999685-0.54269999266-1.7663999796$

$\mathrm{H}-3.3155999184-0.93839997053-0.68049997091$

$\mathrm{H}-4.0911002159-0.28880000114-2.1110000610$

$\mathrm{H}-2.3078000546-2.7820999622-2.0388998985$

$\mathrm{H}-4.0633001328-2.7723000050-2.1731998920$

$\mathrm{H}-2.9453001022-3.0789999962-4.4211001396$

$\mathrm{H}-3.9356999397-1.6225999594-4.3653001785$

H $-0.89029997587-1.7545000315-4.0413999557$

$\mathrm{H}-1.7575000525-1.0898000002-5.4211001396$

$\mathrm{H}-2.6881999969 \quad 0.73869997263-4.0184001923$

$\mathrm{H}-0.92949998379 \quad 0.69330000877-3.9305000305$

$\mathrm{H}-1.27820003032 .6259000301-3.1805000305$

$\mathrm{H}-1.9402999878 \quad 4.5901999474 \quad-1.8831000328$

H $-0.46700000763 \quad 4.4855999947-0.90869998932$

$\mathrm{H}-0.63109999895 \quad 4.9320001602-3.9326999187$

$\mathrm{H}-0.16640000045 \quad 6.1304998398-2.7313001156$

H $1.84159994135 .1455998421-3.8954000473$

H $1.8734999895 \quad 4.8376998901-2.1621999741$

H $0.99659997225 \quad 2.8531999588-4.3302001953$

H $2.46659994132 .7569999695-3.3649001122$

H $1.13759994512 .4688999653-1.2956000566$

H $0.72920000553 \quad 1.2222000360-2.4684000015$

H $0.12479999661 \quad 1.0140999556-0.050599999726$

$\mathrm{H}-0.44029998779 \quad 3.2723999023 \quad 0.88010001183$

$\mathrm{H}-1.7666000128 \quad 2.5515999794 \quad 1.7932000160$

$\mathrm{H} 1.2180999517 \quad 1.9126000404 \quad 2.1170001030$

H $0.235200002792 .8531000614 \quad 3.2346999645$

H $0.53310000896 \quad 0.47330000997 \quad 4.0187001228$

$\mathrm{H}-1.1626000404 \quad 0.86690002680 \quad 3.7432999611$

H $0.66769999266-0.66390001774 \quad 1.8179999590$

$\mathrm{H}-0.65960001945-1.3496999741 \quad 2.7495999336$

H $-2.3136999607 \quad 0.013899999671 \quad 1.4929000139$

$\mathrm{H}-1.3265000582-0.94929999113 \quad 0.39789998531$

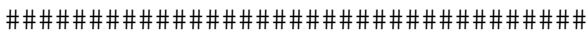

C4C4C4_EE

91

Ru $22.799200058-8.8938999176-0.53729999065$

C $21.961200714-8.9371995926-2.3487000465$

C $20.718900681-8.5682001114-1.4335999489$

C $20.948499680-8.56659984590 .15000000596$

Cl $22.352699280-11.308099747-0.40180000663$

C1 $23.274700165-6.4818000793-0.55080002546$

H $21.883600235-9.9673004150-2.7065999508$

H $20.479200363-7.5299000740-1.6778000593$ 
H $20.573299408-9.49250030520 .59450000525$

C $19.545600891-9.5374002457-1.7217999697$

C $19.020599365-9.4609003067-3.1614000797$

C $17.799499512-10.362099648-3.3761000633$

H $19.873800278-10.554900169-1.4848999977$

H $18.725400925-9.2933998108-1.0341000557$

H $18.758300781-8.4214000702-3.4031999111$

H $19.812000275-9.7516002655-3.8626999855$

H $18.044799805-11.410499573-3.1733000278$

H $16.975900650-10.079299927-2.7105998993$

P $25.054700851-9.36660003660 .29769998789$

C $25.068399429-9.7585000992 \quad 2.1328999996$

C $24.536600113-8.5642995834 \quad 2.9549000263$

C $24.644800186-8.83500003814 .4647002220$

C $23.922100067-10.1323995594 .8550000191$

C $24.436300278-11.3194999694 .0274000168$

C $24.325099945-11.056599617 \quad 2.5143001080$

$\begin{array}{llll}\text { C } 26.236299515 & -7.9162001610 & 0.12630000710\end{array}$

C $27.548099518-7.9752001762 \quad 0.94069999456$

C $28.312099457-6.6419000626 \quad 0.82550001144$

C $28.586799622-6.2642998695-0.63760000467$

C $27.286500931-6.2344999313-1.4538999796$

C $26.521600723-7.5644998550-1.3490999937$

C $25.705900192-10.913499832-0.53490000963$

C $25.519100189-10.922300339-2.0676000118$

C $25.852399826-12.311100006-2.6391000748$

C $27.269699097-12.761099815-2.2518999577$

C $27.472400665-12.716799736-0.72939997911$

C $27.143999100-11.325599670-0.15600000322$

H $26.130899429-9.8969001770 \quad 2.3782000542$

H $25.069999695 \quad-7.6419000626 \quad 2.7019999027$

H $23.486099243-8.3964996338 \quad 2.6912000179$

H $25.706300735-8.90950012214 .7435002327$

H $24.234500885-7.98280000695 .0198998451$

H $24.046600342-10.3309001925 .9264001846$

H $22.843799591-10.010399818 \quad 4.6792998314$

H $25.488000870-11.512499809 \quad 4.2860999107$

H $23.880100250-12.2305002214 .2786998749$

H $23.274099350-10.984399796 \quad 2.2158999443$

H $24.735399246-11.911499977 \quad 1.9680999517$

H $25.623600006-7.09469985960 .51959997416$

H $27.348400116-8.1807003021 \quad 1.9974000454$

H $28.187799454-8.78740024570 .57539999485$

H $27.716800690-5.84840011601 .2987999916$

H $29.251300812-6.70930004121 .3884999752$

H $29.093700409-5.2930002213-0.68879997730$

H $29.275199890-7.0012998581-1.0772999525$

H $26.637199402-5.4274997711-1.0892000198$

H $27.500900269-6.0159997940-2.5072000027$

H $27.121500015-8.3587999344-1.8128000498$

H $25.579000473-7.4864997864-1.8971999884$

H $25.007400513-11.663999557-0.14509999752$

H $24.486600876-10.662799835-2.3152000904$

H $26.170299530-10.173299789-2.5346999168$

H $25.118099213-13.033499718-2.2574000359$

H $25.742399216-12.295800209-3.7304000854$

H $27.466600418-13.771400452-2.6308000088$

H $28.004100800-12.097299576-2.7314999104$

H $26.821199417-13.462900162-0.25290000439$

H $28.503900528-12.987299919-0.47130000591$

H $27.856899261-10.597299576-0.56290000677$

H $27.281400681-11.3318996430 .93150001764$

H $17.434499741-10.296299934-4.4063000679$

$\begin{array}{lllll}\text { C } 20.427499771 & -7.3310999870 & 0.85399997234\end{array}$

C $20.826200485-7.24060010912 .3320999146$

C $20.122299194-6.0894999504 \quad 3.0580000877$

H $19.325899124-7.3758997917 \quad 0.78990000486$

H $20.751800537-6.42789983750 .33250001073$

H $20.604000092-8.1906995773 \quad 2.8378999233$

H $21.910100937 \quad-7.0945000648 \quad 2.3847999573$

H $20.356599808 \quad-5.1293001175 \quad 2.5852999687$ 


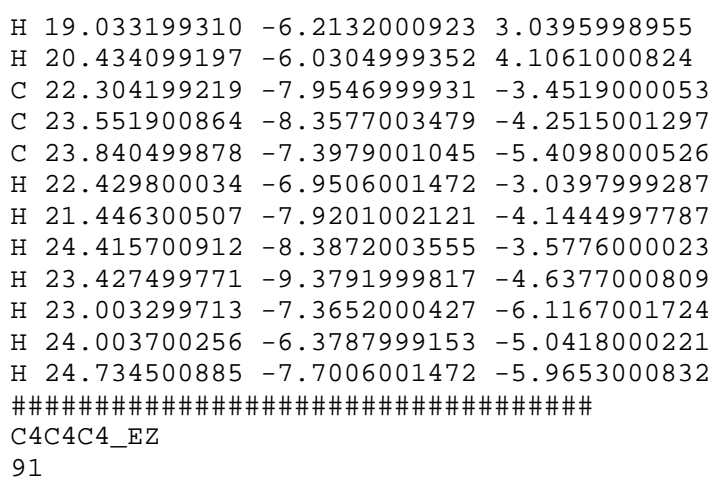


$\begin{array}{llll}C & -0.58829998970 & 6.8123998642 & 0.88760000467\end{array}$

C $0.50800001621 \quad 6.6547999382 \quad 1.9510999918$

C $1.3056999445 \quad 5.3516001701 \quad 1.7641999722$

H $3.4024999142 \quad 3.56669998172 .3069000244$

H $4.1072998047 \quad 1.3934999704 \quad 1.4198999405$

H $5.53900003432 .1254999638 \quad 0.68229997158$

H $4.8561000824 \quad 2.0457999706 \quad 3.6637001038$

H $6.12830019001 .1227999926 \quad 2.8682000637$

H $7.05410003663 .1545000076 \quad 4.0405001640$

H 7.35760021213 .20120000842 .3064000607

H $5.1308999062 \quad 4.70270013813 .7878000736$

H $6.56400012975 .4246001244 \quad 3.0594000816$

H $5.8116998672 \quad 4.7308001518 \quad 0.80150002241$

H $4.5700998306 \quad 5.70289993291 .5870000124$

H $2.9168999195 \quad 3.9326000214-2.3024001122$

H $3.7307000160 \quad 6.2020998001-1.5752999783$

H $5.28179979325 .5015997887-1.1275000572$

H $4.0387001038 \quad 5.5355000496-3.9272999763$

H $5.40659999856 .5051999092-3.3857998848$

H $6.1493000984 \quad 4.4993000031-4.7165999413$

H $6.7824997902 \quad 4.4647998810-3.0734000206$

$\mathrm{H} \quad 4.34709978102 .8943998814-4.0932002068$

H $5.91680002212 .2172999382-3.6658000946$

H $5.60120010382 .8912000656-1.3040000200$

H $4.23400020601 .9031000137-1.8452999592$

H $2.6112000942 \quad 6.0984997749 \quad 0.22669999301$

H $1.2260999680 \quad 5.4067997932-1.7388000488$

H $0.11469999701 \quad 4.5699000359-0.67820000648$

H $0.66790002584 \quad 7.5890002251-0.69429999590$

H $-0.79049998522 \quad 6.7951998711-1.2815999985$

$\mathrm{H}-1.1196000576 \quad 7.7621002197 \quad 1.0241999626$

$\mathrm{H}-1.3330999613 \quad 6.0135002136 \quad 1.0155999660$

H $1.1940000057 \quad 7.5128002167 \quad 1.8923000097$

H $0.072099998593 \quad 6.6711997986 \quad 2.9572999477$

H $0.66320002079 \quad 4.4842000008 \quad 1.9507999420$

H $2.1012001038 \quad 5.3043999672 \quad 2.5150001049$

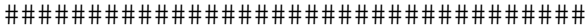

C4C4C4_ZZ

91

C $0.043400000781-0.046399999410-0.045899998397$

C $1.6389000416-0.148499995470 .11529999971$

$\mathrm{Ru} 1.35469996931 .8234000206-0.016000000760$

Cl $1.4338999987 \quad 1.9069999456-2.4551999569$

Cl $1.38170003891 .7414000034 \quad 2.4609000683$

C $-0.57169997692 \quad 1.45840001110 .16629999876$

C $2.5227999687-0.86330002546-0.89969998598$

C $3.1559000015-2.1449000835-0.32609999180$

C $4.0479001999-2.8610000610-1.3446999788$

C $-1.5729999542 \quad 2.0294001102-0.82109999657$

C $-3.0344998837 \quad 1.6877000332-0.46500000358$

C $-4.0310001373 \quad 2.3166999817-1.4436000586$

C $-0.53070002794-0.76660001278-1.2760000229$

C $-0.55360001326-2.2946999073-1.1189999580$

C $-1.1880999804-2.9876999855-2.3294000626$

$\mathrm{H}-0.91070002317 \quad 1.5527000427 \quad 1.2000000477$

$\mathrm{H}-0.28929999471-0.50540000200 \quad 0.89039999247$

H $1.8806999922-0.429199993611 .1405999660$

H $1.9867000580-1.0705000162-1.8292000294$

H $3.3327999115-0.18760000169-1.1979999542$

H $3.7448000908-1.88310003280 .56319999695$

H $2.3708000183-2.82780003550 .022800000384$

H $4.5051999092-3.7579998970-0.91460001469$

H $3.4742000103-3.1675000191-2.2267999649$

H $4.8557000160-2.2056000233-1.6900999546$

$\mathrm{H}-1.4701000452 \quad 3.1231000423-0.78769999743$

$\mathrm{H}-1.33080005651 .7415000200-1.8467999697$

$\mathrm{H}-3.1730999947 \quad 0.59890002012-0.44789999723$

H $-3.2441999912 \quad 2.0359001160 \quad 0.55529999733$

H $-5.0641999245 \quad 2.0787000656-1.1698000431$

$\mathrm{H}-3.9312000275 \quad 3.4080998898-1.4589999914$ 
$\mathrm{H}-3.8624999523 \quad 1.9559999704 \quad-2.4644999504$

$\mathrm{H}-1.5621000528-0.43959999084-1.4357000589$

H $0.019799999893-0.46939998865-2.1730000973$

H $0.46050000191-2.6800999641-0.97079998255$

$\mathrm{H}-1.1148999929-2.5543999672-0.21040000021$

$\mathrm{H}-1.2130999565-4.0745000839-2.1989998817$

$\mathrm{H}-2.2174000740-2.6456000805-2.4874999523$

H $-0.62540000677-2.7727999687-3.2448999882$

P $2.7123999596 \quad 3.8401000500 \quad 0.17599999905$

C $2.6954998970 \quad 4.8470997810-1.4062999487$

C $1.3035000563 \quad 5.4331998825-1.7260999680$

C $1.27600002295 .9979000092-3.1561999321$

C $2.3764998913 \quad 7.0486998558-3.3689999580$

C $3.7607998848 \quad 6.4893999100-3.0060000420$

C $3.79119992265 .9215998650-1.5737999678$

C $2.2453999519 \quad 4.85970020291 .6694999933$

C $3.05150008206 .1574997902 \quad 1.8817000389$

C $2.7160999775 \quad 6.7737002373 \quad 3.2527999878$

C 1.20790004737 .02810001373 .4019000530

C 0.395200014115 .74959993363 .1433000565

C $0.72810000181 \quad 5.1287999153 \quad 1.7760000229$

C $4.5155000687 \quad 3.3822999001 \quad 0.41800001264$

$\begin{array}{lllll}\text { C } 4.7944998741 & 2.6742999554 & 1.7617000341\end{array}$

C $6.29239988332 .3515000343 \quad 1.9098999500$

C $6.8168001175 \quad 1.5164999962 \quad 0.73269999027$

C $6.53289985662 .2142999172-0.60530000925$

C $5.03840017322 .5385000706-0.76670002937$

H $2.8717999458 \quad 4.0619001389-2.1524999142$

H $0.53769999743 \quad 4.6584000587-1.6215000153$

$\mathrm{H} \quad 1.0652999878 \quad 6.2375998497-1.0178999901$

H $1.41219997415 .1673998833-3.8617999554$

H $0.28990000486 \quad 6.4313998222-3.3636000156$

H $2.3721001148 \quad 7.4012999535-4.4075999260$

H $2.16659998897 .9257001877-2.7390000820$

H $4.02510023125 .6890001297-3.7114000320$

H $4.5281000137 \quad 7.2666001320-3.1096999645$

H $3.6370999813 \quad 6.7389001846-0.85909998417$

H $4.7849001884 \quad 5.5075998306-1.3710999489$

H $2.4821999073 \quad 4.1606001854 \quad 2.4804000854$

H $4.12960004815 .9704999924 \quad 1.8121000528$

H $2.80310010916 .8826999664 \quad 1.0965000391$

H $3.0504999161 \quad 6.0878000259 \quad 4.0437998772$

$\mathrm{H} 3.2776999474 \quad 7.7067999840 \quad 3.3859000206$

H $0.98860001564 \quad 7.4240999222 \quad 4.4008998871$

H $0.90259999037 \quad 7.8028001785 \quad 2.6830999851$

$\mathrm{H} 0.61030000448 \quad 5.0090999603 \quad 3.9254999161$

$\mathrm{H}-0.67909997702 \quad 5.9640998840 \quad 3.1989998817$

H $0.40790000558 \quad 5.81349992750 .98100000620$

$\mathrm{H} \quad 0.17980000377 \quad 4.1909999847 \quad 1.6527999640$

H $5.0585999489 \quad 4.3383002281 \quad 0.42129999399$

H $4.47200012213 .2987000942 \quad 2.6006999016$

H 4.20090007781 .75610005861 .8252999783

H $6.8629999161 \quad 3.2901999950 \quad 1.9694999456$

H 6.45779991151 .82449996472 .8571999073

H 7.89169979101 .32959997650 .84500002861

H $6.3235998154 \quad 0.533800005910 .73900002241$

H $7.11350011833 .1468999386-0.66060000658$

H $6.86359977721 .5900000334-1.4442000389$

H $4.4675002098 \quad 1.6033999920-0.81779998541$

H $4.86920022963 .0499000549-1.7195999622$

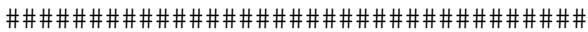

C4C4C_E

82

$\mathrm{Ru} 13.243300438-6.2586998940-0.028599999845$

C $12.413599968-7.1226000786-1.6107000113$

C $11.751999855-5.7007999420-1.6694999933$

C $12.016799927-4.7466998100-0.39079999924$

Cl $11.458299637-7.22149991991 .3533999920$

Cl $15.033300400-5.4821000099-1.5077999830$

H $11.718000412-7.9281001091-1.3795000315$ 
H $12.225500107-5.1757998466 \quad-2.5030999184$

H $11.154999733 \quad-4.7825999260 \quad 0.28119999170$

C $10.218400002-5.8241000175-1.8214000463$

C $9.7979001999-6.4601998329-3.1526000500$

C $8.2751998901-6.5032000542-3.3159999847$

H $13.100899696-7.3133001328-2.4330000877$

H $9.8317003250-6.4021000862-0.97469997406$

H $9.7804002762-4.8196997643-1.7536000013$

H $10.242400169-5.8941001892-3.9828000069$

H $10.204299927-7.4769001007-3.2202999592$

H $7.8095002174-7.0857000351-2.5132999420$

H $7.8453001976-5.4952001572-3.2860000134$

P $14.884400368-6.8987998962 \quad 1.6410000324$

C $15.423199654-8.6592998505 \quad 1.2841000557$

C $14.244999886-9.6556997299 \quad 1.3693000078$

C $14.726900101-11.0975999831 .1368000507$

C $15.466400146-11.238900185-0.20149999857$

C $16.629600525-10.241000175-0.29469999671$

C $16.161199570-8.7933998108-0.067000001669$

C $14.154299736-6.9352998734 \quad 3.3640000820$

C $14.991100311-7.6231999397 \quad 4.4642000198$

C $14.168899536-7.7435998917 \quad 5.7617001534$

C $13.650400162-6.37629985816 .2332000732$

C $12.849200249-5.6718997955 \quad 5.1275000572$

C $13.667499542-5.54650020603 .8315000534$

C $16.451799393-5.8791999817 \quad 1.5306999683$

C $16.209100723-4.3579001427 \quad 1.6292999983$

C $17.495100021-3.5862998962 \quad 1.2849999666$

C $18.673900604-4.0188999176 \quad 2.1703000069$

C $18.895799637-5.53730010992 .0987000465$

C $17.612300873-6.31209993362 .4516999722$

H $16.136299133 \quad-8.9056997299 \quad 2.0845000744$

H $13.742799759-9.5839004517 \quad 2.3389999866$

H $13.489700317-9.39000034330 .62089997530$

H $15.399000168-11.3927001951 .9562000036$

H $13.868399620-11.778699875 \quad 1.1758999825$

H $15.833000183-12.264599800-0.32919999957$

H $14.762299538-11.050299644-1.0242999792$

H $17.388700485-10.4977998730 .45899999142$

H $17.122299194-10.313699722-1.2717000246$

H $15.495699883-8.4823999405-0.88069999218$

$\mathrm{H} 17.023599625-8.1208000183-0.10800000280$

H $13.250200272-7.5324997902 \quad 3.1902999878$

H $15.318900108-8.6190996170 \quad 4.1455998421$

H $15.897500038-7.04190015794 .6708002090$

H $13.316800117-8.4145002365 \quad 5.5840997696$

H $14.781900406-8.2116003036 \quad 6.5419998169$

$\mathrm{H} 13.035799980-6.4949002266 \quad 7.1338000298$

H $14.505900383-5.7462000847 \quad 6.5177998543$

H $11.934000015-6.2396998405 \quad 4.9131999016$

H $12.533499718 \quad-4.6757998466 \quad 5.4611001015$

H $14.527799606-4.8906998634 \quad 4.0152997971$

H $13.057399750-5.08050012593 .0510001183$

H $16.742500305-6.06440019610 .48910000920$

H $15.413900375-4.06920003890 .93779999018$

H $15.890600204-4.08900022512 .6440999508$

H $17.739900589-3.76570010190 .22939999402$

H $17.315799713-2.50929999351 .3887000084$

H $19.587699890-3.4883999825 \quad 1.8761999607$

H $18.468000412-3.73489999773 .2128000259$

H $19.212999344 \quad-5.8122000694 \quad 1.0829000473$

H $19.705099106-5.8399000168 \quad 2.7748000622$

H $17.353799820-6.10739994053 .4976999760$

H $17.798999786-7.3895998001 \quad 2.3759000301$

H $7.9906997681-6.9590001106-4.2698998451$

C $12.464200020-3.3322999477-0.69290000200$

C $12.670599937-2.5055999756 \quad 0.58710002899$

C $13.110600471-1.0677000284 \quad 0.29760000110$

H $11.673600197-2.8494999409-1.2937999964$

H $13.374500275-3.3376998901-1.2979999781$

H $11.741200447-2.50110006331 .1726000309$ 
H $13.423600197 \quad-2.9986000061 \quad 1.2139999866$

H $14.060199738-1.0507999659-0.24889999628$

H $12.368399620-0.53939998150-0.31209999323$

H $13.246899605-0.49959999323 \quad 1.2237999439$

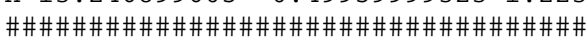

C4C4C_Z

82

Ru $13.175600052-6.27169990540 .079700000584$

C $12.308500290-7.1461000443-1.4601000547$

C $11.695300102-5.6869997978-1.5701999664$

C $12.297100067-4.5963997841-0.53140002489$

Cl $11.377200127-7.06949996951 .5345000029$

Cl $14.995900154-5.6465001106-1.4500999451$

H $11.572400093-7.9021000862-1.1879999638$

H $12.100299835-5.3189001083-2.5178999901$

H $13.001299858-3.9539999962-1.0642000437$

C $10.158100128-5.7375001907-1.5500999689$

C $9.5669002533-6.4604001045-2.7692999840$

C $8.0346002579-6.4467000961-2.7585000992$

H $12.963899612-7.4049000740-2.2899000645$

H $9.8306999207-6.2232999802-0.62449997663$

H $9.7658004761-4.7136001587-1.5319999456$

H $9.9341001511-5.9844999313-3.6898000240$

H $9.9230003357-7.4979000092-2.7967000008$

$\mathrm{H} 7.6440000534-6.9447999001-1.8638000488$

H $7.6477999687-5.4208002090-2.7611999512$

P $14.857000351-6.9642000198 \quad 1.6930999756$

C $15.480899811-8.6644001007 \quad 1.2039999962$

C $14.307399750-9.6649999619 \quad 1.0880000591$

C $14.816200256-11.0817003250 .77380001545$

C $15.681500435-11.100399971-0.49459999800$

C $16.840599060-10.099100113-0.38479998708$

C $16.342899323-8.6752004623-0.076800003648$

C $14.167499542-7.19490003593 .4254999161$

C $15.012200356-8.0293998718 \quad 4.4152002335$

C $14.223199844-8.2798995972 \quad 5.7149000168$

C $13.752400398-6.9678997993 \quad 6.3597998619$

C $12.939999580-6.12340021135 .3670997620$

C $13.725899696-5.8642997742 \quad 4.0706000328$

C $16.340200424-5.8281998634 \quad 1.6565999985$

C $15.963899612-4.3323001862 \quad 1.7409000397$

C $17.200500488-3.4549999237 \quad 1.4795000553$

C $18.353200912-3.7943000793 \quad 2.4375000000$

$\begin{array}{lllll}\text { C } 18.706699371 & -5.2885999680 & 2.3784999847\end{array}$

C $17.472999573-6.1704001427 \quad 2.6465001106$

H $16.120700836 \quad-8.9828996658 \quad 2.0390999317$

H $13.709300041-9.67619991302 .0048000813$

H $13.631999969-9.3326997757 \quad 0.28909999132$

H $15.408399582-11.450300217 \quad 1.6241999865$

H $13.962400436-11.7617998120 .66900002956$

H $16.066400528-12.110600471-0.67940002680$

H $15.057700157-10.836799622-1.3605999947$

H $17.525100708-10.4252004620 .41229999065$

H $17.424999237-10.085399628-1.3126000166$

H $15.762800217-8.2835998535-0.91909998655$

H $17.204200745-8.00879955290 .034299999475$

H $13.245200157-7.7490000725 \quad 3.2098999023$

H $15.292799950-8.9931001663 \quad 3.9781999588$

H $15.945500374 \quad-7.5072999001 \quad 4.6574997902$

H $13.349100113-8.9054002762 \quad 5.4858999252$

H $14.844499588-8.8513002396 \quad 6.4156999588$

$\mathrm{H} 13.158499718-7.1791000366 \quad 7.2572999001$

$\mathrm{H} 14.630000114 \quad-6.3937001228 \quad 6.6918997765$

H $12.007100105-6.64459991465 .1139001846$

H $12.655099869-5.1670999527 \quad 5.8225998878$

H $14.605699539-5.24940013894 .3018999100$

H $13.100999832 \quad-5.3015999794 \quad 3.3705000877$

H $16.700599670 \quad-5.98780012130 .63279998302$

H $15.190099716-4.1073999405 \quad 1.0018999577$

H $15.557700157-4.0974998474 \quad 2.7328000069$ 
H $17.526199341-3.60710000990 .44159999490$

H $16.926900864-2.3968999386 \quad 1.5721000433$

H $19.236000061-3.18729996682 .2033998966$

H $18.058000565-3.5357000828 \quad 3.4651000500$

H $19.110399246-5.5289998055 \quad 1.3849999905$

H $19.493600845-5.5262999535 \quad 3.1048998833$

H $17.131200790 \quad-5.9966998100 \quad 3.6745998859$

H $17.750299454 \quad-7.2288999557 \quad 2.5785000324$

H $7.6244997978-6.9604001045-3.6343998909$

C $11.363499641-3.81360006330 .36710000038$

C $12.138299942-2.90960001951 .3415999413$

C $11.211899757-2.05800008772 .2147998810$

H $10.732700348-3.1677000523-0.26719999313$

H $10.699199677-4.4784998894 \quad 0.92320001125$

H $12.771499634-3.53620004651 .9814000130$

H $12.820899963-2.2595999241 \quad 0.77679997683$

H $10.584799767-1.39890003201 .6030000448$

H $10.545100212 \quad-2.6903998852 \quad 2.8118999004$

H $11.783399582-1.42830002312 .9051001072$

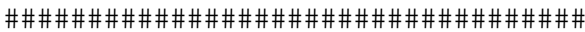

$\mathrm{C} 4 \mathrm{CC}$

73

$\mathrm{Ru} 0.83190000057-0.25839999318 \quad 1.4722000360$

C $1.3637000322-2.0924999714 \quad 1.9974000454$

C $-0.19230000675-2.1268000603 \quad 2.2144999504$

C $-1.0456000566-0.855599999431 .7295000553$

Cl $1.20500004290 .44330000877 \quad 3.7837998867$

Cl $0.60500001907-1.0757999420-0.83029997349$

$\mathrm{H} 1.9286999702-2.1524000168 \quad 2.9261000156$

$\mathrm{H}-0.56230002642-2.94190001491 .5900000334$

$\mathrm{H}-0.38609999418 \quad-2.2476999760 \quad 3.2799999714$

$\mathrm{H}-1.5227999687-1.0664000511 \quad 0.77009999752$

C $-1.9859999418-0.23960000277 \quad 2.7423999310$

C $-2.6517000198 \quad 1.0419000387 \quad 2.2163000107$

C -3.66249990461 .62740004063 .2058999538$

$\mathrm{H} 1.7092000246-2.7290000916 \quad 1.1849000454$

$\mathrm{H}-2.7767000198 \quad-0.97869998217 \quad 2.9586999416$

$\mathrm{H}-1.4601999521-0.034899998456 \quad 3.6784000397$

$\mathrm{H}-1.8727999926 \quad 1.78419995312 .0046999454$

$\mathrm{H}-3.1461999416 \quad 0.83319997787 \quad 1.2575999498$

$\mathrm{H}-4.4745001793 \quad 0.91979998350 \quad 3.4103000164$

$\mathrm{H}-3.1821000576 \quad 1.8662999868 \quad 4.1612000465$

P 2.00300002101 .69490003590 .61790001392

$\begin{array}{lllll}\text { C } 3.7755000591 & 1.2094000578 & 0.24429999292\end{array}$

C $4.44710016250 .58890002966 \quad 1.4911999702$

C $5.92749977110 .27270001173 \quad 1.2224999666$

C $6.0960001945-0.63580000401-0.0038999998942$

C $5.4180002213-0.025499999523-1.2391999960$

$\begin{array}{lllll}\text { C } 3.9326999187 & 0.28790000081 & -0.98379999399\end{array}$

C $2.1524999142 \quad 3.0755000114 \quad 1.8794000149$

C $3.22180008894 .1579999924 \quad 1.6123000383$

C $3.3454999924 \quad 5.0989999771 \quad 2.8259999752$

C $1.9969999790 \quad 5.7325000763 \quad 3.1981000900$

$\begin{array}{lllll}\text { C } 0.92449998856 & 4.6581997871 & 3.4303998947\end{array}$

C $0.79229998589 \quad 3.7207000256 \quad 2.2184998989$

C $1.2661000490 \quad 2.2718999386-0.99830001593$

C $-0.26800000668 \quad 2.4451000690-0.94599997997$

C $-0.82370001078 \quad 2.6939001083-2.3587000370$

C $-0.14980000257 \quad 3.8994998932-3.0322999954$

C $1.37899994853 .7472000122-3.0522999763$

C $1.9398000240 \quad 3.5030000210-1.6390000582$

H 4.28599977492 .15619993210 .017500000075

$\mathrm{H} \quad 4.3571000099 \quad 1.2527999878 \quad 2.3571000099$

H $3.9186999798 \quad-0.33460000157 \quad 1.7599999905$

H 6.47370004651 .21309995651 .0571000576

H $6.3688001633-0.192900002002 .1119000912$

H $7.1596999168-0.81499999762-0.20200000703$

H $5.6447000504-1.6153999567 \quad 0.20810000598$

H $5.94210004810 .90079998970-1.5182000399$

H $5.5026001930-0.70289999247-2.0973000526$ 
H $3.3684000969-0.63770002127-0.82899999619$

H $3.5053999424 \quad 0.75910001993-1.8750000000$

H 2.45230007172 .50990009312 .7708001137

H $4.1988000870 \quad 3.7065000534 \quad 1.4100999832$

H $2.9553999901 \quad 4.74760007860 .72670000792$

H $3.7262001038 \quad 4.5261001587 \quad 3.6831998825$

H $4.0893998146 \quad 5.8762998581 \quad 2.6112999916$

H $2.1080999374 \quad 6.3607997894 \quad 4.0901999474$

H $1.6739000082 \quad 6.3977999687 \quad 2.3836998940$

H $1.1857999563 \quad 4.0576000214 \quad 4.3119001389$

$\mathrm{H}-0.045000001788 \quad 5.1255998611 \quad 3.6417999268$

H $0.42710000277 \quad 4.2973999977 \quad 1.3585000038$

H $0.054900001734 \quad 2.9426000118 \quad 2.4361999035$

H $1.4471999407 \quad 1.3956999779-1.6334999800$

$\mathrm{H}-0.72619998455 \quad 1.5465999842 \quad-0.52289998531$

$\mathrm{H}-0.53339999914 \quad 3.2906000614 \quad-0.29949998856$

$\mathrm{H}-0.65930002928 \quad 1.7928999662 \quad-2.9649999142$

$\mathrm{H}-1.90880000592 .8447000980-2.3059000969$

$\mathrm{H}-0.53020000458 \quad 4.0279998779-4.0528001785$

$\mathrm{H}-0.41200000048 \quad 4.8151998520-2.4821000099$

H $1.65310001372 .9007999897-3.6974999905$

H $1.8482999802 \quad 4.6392002106-3.4855999947$

H $1.7483999729 \quad 4.3913002014-1.0238000154$

H $3.0281000137 \quad 3.3780000210-1.6864999533$

H $-4.1125001907 \quad 2.5471999645 \quad 2.8171000481$

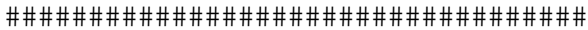

C4CC4_Z

82

$\mathrm{Ru} 26.181299210-6.6350998878 \quad 0.28690001369$

C $25.528099060-6.7362999916-1.6013000011$

C $26.106100082-5.2621002197-1.4883999825$

C $26.980499268-4.8850002289-0.20790000260$

Cl $24.084600449-5.7175998688 \quad 1.1612999439$

Cl $28.258300781-7.6332998276-0.59850001335$

H $26.814300537-5.1901998520-2.3162000179$

H $28.045099258-4.9822001457-0.43180000782$

H $26.188199997-7.3642997742-2.2025001049$

P $26.361099243-8.1380996704 \quad 2.1967000961$

C $25.608100891-9.79249954221 .7358000278$

C $24.127199173-9.63230037691 .3207999468$

C $23.489500046-10.9937000270 .99739998579$

C $24.283500671-11.748900414-0.078199997544$

$\begin{array}{llll}\text { C } 25.755399704 & -11.902400017 & 0.33149999380\end{array}$

C $26.406400681-10.544099808 \quad 0.64829999208$

C $25.359399796-7.5595998764 \quad 3.6719999313$

C $25.073400497-8.5959997177 \quad 4.7800998688$

C $24.067800522-8.02149963385 .7965002060$

C $24.556299210-6.6925001144 \quad 6.3930001259$

C $24.886100769-5.6735000610 \quad 5.2913999557$

C $25.893299103-6.2441000938 \quad 4.2786998749$

$\begin{array}{lllll}\text { C } 28.142599106 & -8.4933004379 & 2.6375999451\end{array}$

C $28.994600296-7.21829986572 .8269000053$

$\begin{array}{lllll}\text { C } 30.483699799 & -7.5879998207 & 2.9367001057\end{array}$

C $30.737899780-8.6006002426 \quad 4.0645999908$

C $29.859300613-9.8513002396 \quad 3.9042999744$

C $28.367500305-9.48270034793 .7997000217$

H $25.642900467-10.3942003252 .6552999020$

H $23.552600861-9.13000011442 .1052999496$

H $24.068599701-8.97999954220 .44040000439$

H $23.450000763-11.6009998321 .9135999680$

H $22.451599121-10.8436002730 .67680001259$

H $23.834299088-12.731900215-0.26469999552$

H $24.228500366-11.192399979-1.0247999430$

H $25.818199158-12.550700188 \quad 1.2180999517$

н $26.324800491-12.401300430-0.46169999242$

H $26.461999893-9.9315004349-0.25819998980$

H $27.440900803-10.7018995290 .96920001507$

H $24.408599854-7.30509996413 .1875000000$

H $24.671300888-9.5240001678 \quad 4.3597998619$

H $25.999799728 \quad-8.8607997894 \quad 5.3035001755$ 


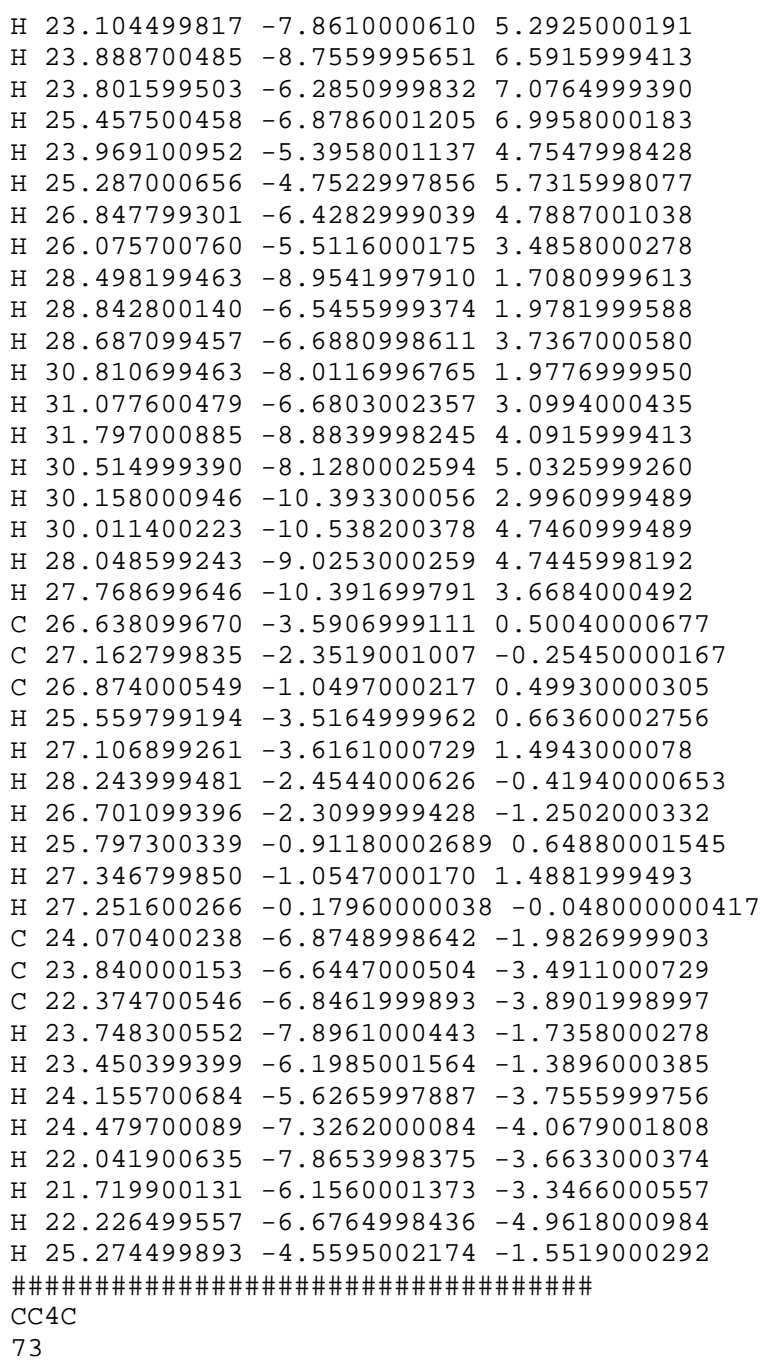




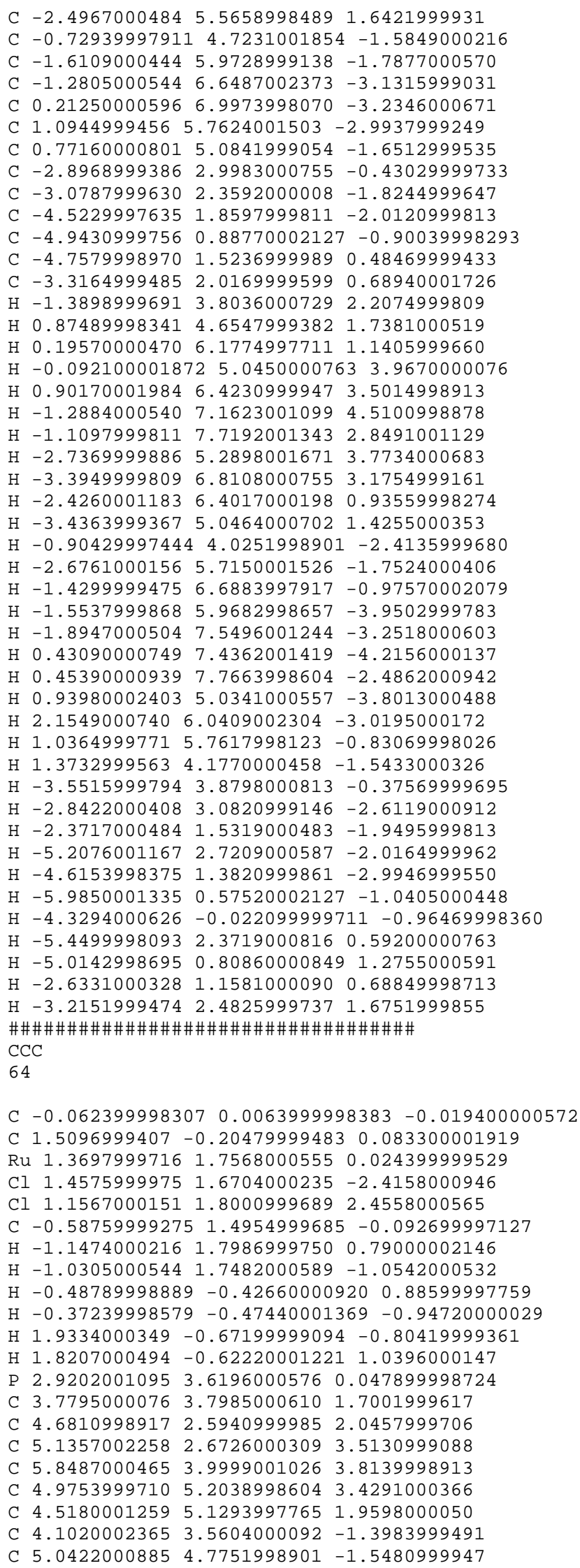


C $5.79570007324 .7051000595-2.8893001080$

C $6.5686998367 \quad 3.3849999905-3.0320999622$

C $5.64610004432 .1712999344-2.8382999897$

C $4.8910999298 \quad 2.2367000580-1.4993000031$

C $1.9321999550 \quad 5.1986999512-0.16560000181$

C $1.28559994705 .3217000961-1.5635000467$

C $0.51800000668 \quad 6.6489000320-1.6969000101$

C $-0.53079998493 \quad 6.8168997765-0.58819997311$

C 0.111100003126 .68650007250 .80040001869

C $0.87400001287 \quad 5.35960006710 .95010000467$

H $2.9210999012 \quad 3.74070000652 .3819000721$

$\mathrm{H} \quad 4.13899993901 .6585999727 \quad 1.8762999773$

H 5.56589984892 .59080004691 .3963999748

H $4.25390005112 .5694999695 \quad 4.1592001915$

H $5.79489994051 .8260999918 \quad 3.7400000095$

H $6.1206998825 \quad 4.0549001694 \quad 4.8748998642$

H $6.7899999619 \quad 4.0426001549 \quad 3.2464001179$

H $4.0886001587 \quad 5.2326998711 \quad 4.0774002075$

H 5.51910018926 .14190006263 .5968999863

H 5.39610004435 .20900011061 .3078999519

$\mathrm{H} 3.8768999577 \quad 5.9886999130 \quad 1.7344000340$

H $3.3966999054 \quad 3.5539000034-2.2388000488$

$\mathrm{H} \quad 4.4861998558 \quad 5.7178997993-1.4845000505$

H $5.7734999657 \quad 4.7821002007-0.73049998283$

H $5.0721998215 \quad 4.7957000732-3.7114999294$

H $6.4773998260 \quad 5.5605001450-2.9735000134$

H $7.0590000153 \quad 3.3387000561-4.0118999481$

H $7.3699002266 \quad 3.3552999496-2.2790000439$

H $4.91149997712 .1305999756-3.6537001133$

H $6.22520017621 .2414000034-2.8889000416$

H $5.61219978332 .1626999378-0.67599999905$

H $4.20450019841 .3888000250-1.4207999706$

H $2.66510009776 .0116000175-0.059900000691$

H $2.0462999344 \quad 5.2620000839-2.3482000828$

H $0.60869997740 \quad 4.4765000343-1.7329000235$

H $1.2305999994 \quad 7.4855999947-1.6509000063$

$\mathrm{H} 0.044500000775 \quad 6.6958999634-2.6847000122$

$\mathrm{H}-1.0361000299 \quad 7.7853999138-0.68529999256$

$\mathrm{H}-1.3044999838 \quad 6.0444998741-0.70260000229$

$\mathrm{H} 0.80629998446 \quad 7.5237998962 \quad 0.95980000496$

$\mathrm{H}-0.65069997311 \quad 6.7586998940 \quad 1.5857000351$

H $0.16840000451 \quad 4.5208001137 \quad 0.90429997444$

$\mathrm{H} \quad 1.33910000325 .3077998161 \quad 1.9395999908$

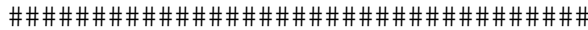

butylidene-P

119

$\mathrm{Ru} \quad 0.94870001078-0.31799998879-0.71689999104$

Cl $0.75599998236-0.29069998860 \quad 1.7500000000$

$\mathrm{Cl} \quad 0.77259999514 \quad-0.49759998918-3.1728999615$

C $2.63420009610 .39530000091-0.77300000191$

P $1.5548000336-2.7016999722-0.67439997196$

H $3.0144999027 \quad 0.64889997244 \quad-1.7753000259$

C 3.59800004960 .709900021550 .33950001001

C $-0.0037000000011-3.7578001022-0.66449999809$

C $2.4530999660-3.2934000492-2.2128999233$

C $2.4644000530-3.24909996990 .88590002060$

C $3.6686000824-2.4321999550-2.6149001122$

C $4.1989002228-2.8578999043-3.9948999882$

C $4.5444002151-4.3541002274-4.0390000343$

C $3.3419001102-5.2123999596-3.6184000969$

C $2.8052000999-4.7964000702-2.2362999916$

C $-0.78460001945-3.7541999817-1.9965000153$

C $-1.9927999973-4.7059998512-1.9178999662$

C $-2.9181001186-4.3698000908-0.73900002241$

C $-2.1417999268-4.32210016250 .58590000868$

C $-0.93949997425-3.3668000698 \quad 0.50019997358$

C $2.2604999542-4.7062001228 \quad 1.3596999645$

C $2.9184999466-4.9250001907 \quad 2.7362000942$

$\begin{array}{lllll}\text { C } 4.4103999138 & -4.5602998734 & 2.7288000584\end{array}$

C $4.6254000664-3.12890005112 .2151999474$ 
C $3.9741001129-2.93000006680 .83590000868$

$\mathrm{H} 0.34060001373-4.7863998413-0.49160000682$

$\mathrm{H} 1.6971000433-3.0987999439-2.9839999676$

H $2.0151000023-2.5834999084 \quad 1.6356999874$

H $3.3689000607-1.3838000298-2.6491000652$

H $4.4721999168-2.5322000980-1.8746000528$

H $3.4316000938-2.6352000237-4.7491998672$

H $5.0785999298-2.2550001144 \quad-4.2536997795$

H $4.8817000389-4.6402001381-5.0430002213$

H $5.3846001625-4.5525999069-3.3568000793$

H $2.5392999649-5.1008000374 \quad-4.3614001274$

H $3.6135001183-6.2755999565-3.6052999496$

H $1.9328000546-5.4092998505-1.9823000431$

H $3.5706999302-5.0097999573-1.4788999557$

$\mathrm{H}-1.1174999475-2.7358000278-2.2302999496$

$\mathrm{H}-0.14290000498-4.0606999397-2.8278999329$

$\mathrm{H}-1.6277999878-5.7381000519-1.8084000349$

$\mathrm{H}-2.5499000549-4.6704998016-2.8622999191$

$\mathrm{H}-3.3814001083-3.3891000748-0.91750001907$

$\mathrm{H}-3.7376999855-5.0963997841-0.67739999294$

$\mathrm{H}-2.8022000790-4.0130000114 \quad 1.4055000544$

H $-1.7836999893-5.3319001198 \quad 0.83569997549$

$\mathrm{H}-0.40310001373-3.34100008011 .4535000324$

$\mathrm{H}-1.2970999479-2.3424000740 \quad 0.34139999747$

H $2.6954998970-5.40520000460 .63330000639$

H $1.1977000237-4.9514999390 \quad 1.4380999804$

H $2.7811000347-5.9682998657 \quad 3.0473001003$

H $2.3977000713-4.3035998344 \quad 3.4783000946$

H $4.9513001442-5.26160001752 .0764000416$

H $4.8348999023-4.6750001907 \quad 3.7337999344$

H $4.1866002083-2.4140000343 \quad 2.9252998829$

H $5.6974000931-2.90089988712 .1558001041$

H $4.1407999992-1.9078999758 \quad 0.48930001259$

H $4.4681000710-3.59330010410 .11400000006$

P $-0.33680000901 \quad 1.7741999626-0.85740000010$

C $-0.154799997812 .6933999062-2.4862999916$

C $-2.1789000034 \quad 1.3863999844-0.79579997063$

C $-0.080099999905 \quad 2.9402999878 \quad 0.60030001402$

C $-2.6960999966 \quad 0.62809997797 \quad-2.0378999710$

C $-4.2203998566 \quad 0.42500001192-1.9573999643$

C $-4.6405000687-0.28799998760-0.66329997778$

C -4.09079980850 .433499991890 .57630002499$

$\begin{array}{lllll}\text { C }-2.5664000511 & 0.62199997902 & 0.48949998617\end{array}$

C $1.3055000305 \quad 2.9595000744 \quad-2.9107000828$

C $1.3588999510 \quad 3.5067000389-4.3477001190$

C $0.49959999323 \quad 4.7690000534-4.5131001472$

C $-0.94840002060 \quad 4.5145001411-4.0676999092$

C $-1.0109000206 \quad 3.9702000618-2.6284000874$

C $1.2184000015 \quad 3.76600003240 .48019999266$

C $1.5437999964 \quad 4.4689998627 \quad 1.8090000153$

C $0.386000007395 .3766999245 \quad 2.2520000935$

C $-0.93790000677 \quad 4.5995998383 \quad 2.3129999638$

C $-1.2469999790 \quad 3.8773000240 \quad 0.98720002174$

$\mathrm{H}-0.526400029661 .9414999485-3.1940000057$

$\mathrm{H}-2.6835000515 \quad 2.3620998859-0.77179998159$

H $0.065899997950 \quad 2.2218000889 \quad 1.4185999632$

$\mathrm{H}-2.4533998966 \quad 1.1682000160 \quad-2.9579999447$

H $-2.1900999546-0.34200000763-2.1147999763$

$\mathrm{H}-4.71439981461 .4067000151-2.0065000057$

$\mathrm{H}-4.5619001389-0.14040000737-2.8334000111$

$\mathrm{H}-5.7333002090-0.36750000715-0.60890001059$

$\mathrm{H}-4.2522001266-1.3162000179-0.67820000648$

$\mathrm{H}-4.5727000237 \quad 1.4182000160 \quad 0.66879999638$

$\mathrm{H}-4.3425002098-0.12479999661 \quad 1.4865000248$

$\mathrm{H}-2.19580006601 .1299999952 \quad 1.3844000101$

$\mathrm{H}-2.0741000175-0.35710000992 \quad 0.48960000277$

H $1.7784999609 \quad 3.6805000305-2.2328999043$

$\mathrm{H} \quad 1.8683999777 \quad 2.0269999504 \quad-2.8610000610$

H $2.40030002593 .7144000530-4.6240000725$

H $1.00129997732 .7256000042-5.0328998566$

H $0.92580002546 \quad 5.5803999901 \quad-3.9042000771$ 
H $0.52109998465 \quad 5.1138000488 \quad-5.5542998314$ $\mathrm{H}-1.5413000584 \quad 5.4348998070-4.1413002014$ $\mathrm{H}-1.4133000374 \quad 3.7843000889-4.7455000877$ $\mathrm{H}-2.0548999310 \quad 3.7788999081 \quad-2.3552000523$ $\mathrm{H}-0.64149999619 \quad 4.7417001724 \quad-1.9402999878$ H $2.0536999702 \quad 3.1303000450 \quad 0.17530000210$

H $1.1004999876 \quad 4.5267000198 \quad-0.30250000954$ H $2.46770000465 .0522999763 \quad 1.7060999870$ H $1.7314000130 \quad 3.70819997792 .5794000626$ H $0.285400003196 .2052001953 \quad 1.5353000164$ H $0.60570001602 \quad 5.8296999931 \quad 3.2265999317$ $\mathrm{H}-0.88270002604 \quad 3.85209989553 .1170001030$ $\mathrm{H}-1.7652000189 \quad 5.2744002342 \quad 2.5668001175$ $\mathrm{H}-2.1828000546 \quad 3.3225998878 \quad 1.0980999470$ H $-1.4114999771 \quad 4.6216998100 \quad 0.19730000198$ C $5.0659999847 \quad 0.75660002232-0.12200000137$ C $6.0258998871 \quad 1.07120001321 .0295000076$ H $3.45219993590 .023700000718 \quad 1.1776000261$ H $3.3234000206 \quad 1.6947000027 \quad 0.74620002508$ H $5.3397002220-0.20379999280-0.57840001583$

H $5.17140007021 .5104000568-0.91380000114$

H $5.9660000801 \quad 0.30579999089 \quad 1.8115999699$

$\mathrm{H} 7.0637998581 \quad 1.1171000004 \quad 0.68339997530$ H $5.7831001282 \quad 2.03439998631 .4931000471$

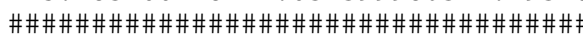

butylidene-eoctene-E-TS 91

C $-0.017100000754 \quad 0.0059000002220-0.0051000001840$ C $1.9816999435-0.058600001037-0.031300000846$

$\mathrm{Ru} 1.5034999847 \quad 1.7842999697 \quad 0.012099999934$

Cl $1.44900000102 .0120000839-2.4467999935$

Cl $1.50610005861 .7223999500 \quad 2.4676001072$

C $-0.53979998827 \quad 1.3811999559 \quad 0.094599999487$

C $2.5873999596-0.77609997988-1.2152999640$

C $2.7671999931-2.2976000309-1.0757999420$

C $3.6282000542-2.8677000999-2.2088000774$

C $-1.44330000881 .9610999823-0.97619998455$

C $-2.9179000854 \quad 1.5750999451-0.73879998922$

C $-3.8512001038 \quad 2.2007999420-1.7807999849$

C $-0.40470001101-0.896399974821 .1734999418$

C $-0.063400000334-2.3842999935 \quad 1.0480999947$

C $-0.70420002937-3.2111999989 \quad 2.1682999134$

$\mathrm{H}-0.85610002279 \quad 1.6374000311 \quad 1.1083999872$

$\mathrm{H}-0.14399999380-0.42370000482-1.0002000332$

$\mathrm{H} 2.1921000481-0.506900012490 .94569998980$

н $2.0548999310-0.51920002699-2.1343998909$

H $3.5868999958-0.33090001345-1.3377000093$

H $3.2314000130-2.5295000076-0.10740000010$

$\mathrm{H} 1.7891000509-2.7904000282-1.0786000490$

H $3.7407000065-3.9528999329-2.1168999672$

H $3.1805999279-2.6579999924-3.1867001057$

H $4.6307997704-2.4247000217-2.2037000656$

$\mathrm{H}-1.36269998553 .0564000607-0.96560001373$

$\mathrm{H}-1.11849999431 .6395000219-1.9678000212$

$\mathrm{H}-3.01570010190 .48140001297-0.76569998264$

$\mathrm{H}-3.2246000767 \quad 1.88740003110 .26870000362$

$\mathrm{H}-4.8942999840 \quad 1.9170999527-1.6052999496$

$\mathrm{H}-3.79229998593 .2950000763-1.7540999651$

H $-3.5810999870 \quad 1.8810000420-2.7934000492$

H $0.019400000572-0.47549998760 \quad 2.0912001133$

$\mathrm{H}-1.4960999489-0.80830001831 \quad 1.2726999521$

H $-0.40470001101-2.76110005380 .073499999940$

H $1.0217000246 \quad-2.5269999504 \quad 1.0729999542$

$\mathrm{H}-0.45030000806-4.2720999718 \quad 2.0745999813$

$\mathrm{H}-0.36259999871-2.8701999187 \quad 3.1521000862$

$\mathrm{H}-1.7968000174-3.1252999306 \quad 2.1493999958$

P 3.04550004013 .69479990010 .069899998605

C 4.04530000693 .75699996951 .6527999640

C $4.9496998787 \quad 2.5199999809 \quad 1.8404999971$

C $5.52220010762 .4883999825 \quad 3.2678000927$ 
C $6.27930021293 .7829999924 \quad 3.6031999588$

C $5.39949989325 .0216999054 \quad 3.3740999699$

C $4.82789993295 .0549001694 \quad 1.9440000057$

C $4.1057000160 \quad 3.8478000164-1.4643000364$

C $4.94199991235 .1380000114-1.5971000195$

C $5.56470012665 .2274999619-3.0028998852$

C $6.4120001793 \quad 3.9860000610-3.3227999210$

C $5.60150003432 .6926000118-3.1431999207$

C $4.97499990462 .6036000252-1.7407000065$

C 2.01740002635 .26809978490 .096000000834

C $1.28079998495 .5307998657-1.2367000580$

C $0.48300001025 \quad 6.8454999924-1.1753000021$

C $-0.49729999900 \quad 6.8653001785 \quad 0.0062000001781$

C $0.23170000315 \quad 6.5945000648 \quad 1.3302999735$

C $1.02470004565 .2775998116 \quad 1.2814999819$

H 3.24760007863 .66050004962 .4001998901

H $4.3762001991 \quad 1.60769999031 .6509000063$

H 5.77920007712 .54990005491 .1220999956

$\mathrm{H} \quad 4.6922998428 \quad 2.34969997413 .9737000465$

H $6.1834001541 \quad 1.62049996853 .3817999363$

H $6.6356000900 \quad 3.7592999935 \quad 4.6402997971$

H $7.1730999947 \quad 3.8552999496 \quad 2.9660000801$

H $4.5665998459 \quad 5.0145998001 \quad 4.0911998749$

H $5.9710998535 \quad 5.93870019913 .5638000965$

H $5.6556000710 \quad 5.16540002821 .2330000401$

$\mathrm{H} 4.1900000572 \quad 5.93800020221 .8272000551$

H $3.3327999115 \quad 3.8505001068-2.2428998947$

H $4.3333001137 \quad 6.0286998749-1.4052000046$

H $5.7476000786 \quad 5.1395998001-0.85269999504$

H $4.75990009315 .3214001656-3.7455000877$

H $6.1730999947 \quad 6.1371998787-3.0804998875$

H $6.8064999580 \quad 4.0500998497-4.3442001343$

H $7.2828001976 \quad 3.9637000561-2.6510000229$

$\mathrm{H} \quad 4.79619979862 .6540000439-3.8891000748$

H $6.23869991301 .8172999620-3.3201999664$

H $5.77449989322 .5255999565-0.99309998751$

H $4.3607997894 \quad 1.7021000385-1.6671999693$

H $2.7358000278 \quad 6.0857000351 \quad 0.24979999661$

H $1.99010002615 .5767998695-2.0687999725$

H $0.60979998112 \quad 4.6933999062 \quad-1.4602999687$

$\mathrm{H} 1.1825000048 \quad 7.6890001297-1.0789999962$

$\mathrm{H}-0.052600000054 \quad 6.9902000427 \quad-2.1214001179$

$\mathrm{H}-1.0242999792 \quad 7.8263001442 \quad 0.049100000411$

$\mathrm{H}-1.2640999556 \quad 6.0924000740-0.14800000191$

$\mathrm{H} \quad 0.92210000753 \quad 7.4246001244 \quad 1.5412000418$

H $-0.48129999638 \quad 6.5615000725 \quad 2.1628000736$

H $0.32940000296 \quad 4.4342999458 \quad 1.1806000471$

H $1.5461000204 \quad 5.1201000214 \quad 2.2306001186$

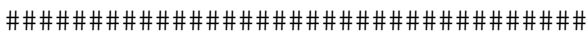

butylidene-eoctene-Z-TS

91

C $0.068999998271 \quad 0.016200000420 \quad-0.10119999945$

C $2.0641999245 \quad 0.0388000011440 .035199999809$

Ru 0.584900021551 .83360004430 .013500000350

Cl $0.606599986551 .8116999865-2.4547998905$

$\mathrm{Cl} \quad 0.63139998913 \quad 1.9788000584 \quad 2.4686000347$

C $2.6285998821 \quad 1.39370000360 .11959999800$

C $3.5088999271 \quad 1.9848999977-0.96509999037$

C $5.0076999664 \quad 1.7222000360-0.71329998970$

$\begin{array}{llll}\text { C } 5.8955998421 & 2.4135999680 & -1.7532999516\end{array}$

C $-0.67890000343-0.77480000257 \quad 0.94220000505$

C $-0.94569998980-2.2376999855 \quad 0.54329997301$

C $-1.7684999704-2.98359990121 .5983999968$

C $2.3520998955-0.888700008391 .2134000063$

C $3.8357000351-1.29410004621 .2774000168$

C $4.1147999763-2.26620006562 .4284999371$

$\mathrm{H}-0.028599999845-0.37500000000-1.1194000244$

H $2.1940999031-0.40020000935-0.95480000973$

H $2.93659996991 .6670999527 \quad 1.1312999725$

H $3.21110010151 .6076999903-1.9457999468$ 
H $3.35330009463 .0724999905-0.99610000849$

H 5.27850008012 .07030010220 .29319998622

H $5.1964001656 \quad 0.64130002260-0.72689998150$

H $6.9566998482 \quad 2.2176001072 \quad-1.5658999681$

H $5.66279983522 .0641000271-2.7653999329$

H $5.7477002144 \quad 3.4997000694 \quad-1.7373000383$

$\mathrm{H}-0.19969999790 \quad-0.69349998236 \quad 1.9212000370$

$\mathrm{H}-1.6483999491-0.26879999042 \quad 1.0628000498$

$\mathrm{H}-1.4721000195-2.2548999786-0.42039999366$

H $0.0048000002280-2.7613000870 \quad 0.37880000472$

H $-1.9648000002-4.01700019841 .2947000265$

$\mathrm{H}-1.2446999550-3.00990009312 .5606000423$

H $-2.7348999977-2.4930999279 \quad 1.7628999949$

H $2.0671999454-0.379200011492 .1398000717$

H $1.7532000542-1.80149996281 .1409000158$

H $4.1342000961-1.75409996510 .32490000129$

H $4.4552998543-0.397500008341 .3954000473$

H $5.1729001999-2.5457000732 \quad 2.4646000862$

H $3.8533000946-1.81710004813 .3931999207$

H $3.5295000076-3.1868999004 \quad 2.3218998909$

P $-0.972100019453 .7200000286-0.073499999940$

C $-2.1238999367 \quad 3.6136999130-1.5400999784$

C $-2.8622000217 \quad 2.2616000175-1.6449999809$

C $-3.5985999107 \quad 2.1603999138-2.9916999340$

C $-4.5627999306 \quad 3.3382000923-3.2042999268$

C $-3.8394999504 \quad 4.6865000725 \quad-3.0622000694$

C $-3.1045999527 \quad 4.7918000221-1.7130999565$

C $-1.8844000101 \quad 3.9949998856 \quad 1.5408999920$

C $-2.5701000690 \quad 5.3625001907 \quad 1.7511999607$

C $-3.0755999088 \quad 5.4865999222 \quad 3.2016000748$

C $-4.0156002045 \quad 4.3313999176 \quad 3.5789999962$

C $-3.3527998924 \quad 2.9681000710 \quad 3.3287000656$

C $-2.85109996802 .8413000107 \quad 1.8803000450$

C $0.0218000002215 .2958998680-0.31859999895$

C $1.0975999832 \quad 5.4345002174 \quad 0.78450000286$

$\begin{array}{lllll}\text { C } 1.8868000507 & 6.7456998825 & 0.63510000706\end{array}$

C $2.5155999660 \quad 6.8682999611-0.76029998064$

C $1.4509999752 \quad 6.7259001732-1.8576999903$

C $0.656599998475 .4140000343-1.7220000029$

$\mathrm{H}-1.4054000378 \quad 3.6249001026-2.3694999218$

$\mathrm{H}-2.1421000957 \quad 1.4441000223-1.5555000305$

$\mathrm{H}-3.58780002592 .1612000465-0.82789999247$

$\mathrm{H}-2.85260009772 .1431000233-3.7978999615$

$\mathrm{H}-4.1424999237 \quad 1.2093000412-3.0448000431$

$\mathrm{H}-5.0409998894 \quad 3.2672998905-4.1887001991$

$\mathrm{H}-5.3699002266 \quad 3.2841999531-2.4586999416$

$\mathrm{H}-3.1096000671 \quad 4.7964000702-3.8764998913$

$\mathrm{H}-4.55060005195 .5159997940-3.1617000103$

$\mathrm{H}-3.8454000950 \quad 4.7785000801-0.90359997749$

$\mathrm{H}-2.5820999146 \quad 5.7534999847 \quad-1.6481000185$

$\mathrm{H}-1.0570000410 \quad 3.9161999226 \quad 2.2574999332$

$\mathrm{H}-1.8808000088 \quad 6.1869997978 \quad 1.5400999784$

$\mathrm{H}-3.4179000854 \quad 5.4749999046 \quad 1.0643999577$

$\mathrm{H}-2.2111001015 \quad 5.4875998497 \quad 3.8803000450$

$\mathrm{H}-3.5810999870 \quad 6.4513998032 \quad 3.3338999748$

$\mathrm{H}-4.3210000992 \quad 4.4204001427 \quad 4.6287999153$

$\mathrm{H}-4.9338998795 \quad 4.4036998749 \quad 2.9776999950$

$\mathrm{H}-2.49839997292 .8408000469 \quad 4.0068998337$

$\mathrm{H}-4.0564999580 \quad 2.1561000347 \quad 3.5495998859$

$\mathrm{H}-3.7116999626 \quad 2.8612000942 \quad 1.1987999678$

$\mathrm{H}-2.3436999321 \quad 1.8811000586 \quad 1.7482000589$

$\mathrm{H}-0.69220000505 \quad 6.1234998703-0.20419999957$

$\mathrm{H} \quad 0.64730000496 \quad 5.3791999817 \quad 1.7807999849$

H $1.7905999422 \quad 4.5845999718 \quad 0.72240000963$

H $1.2102999687 \quad 7.59609985350 .80570000410$

H $2.65860009196 .7986998558 \quad 1.4125000238$

H $3.04150009167 .8256998062-0.85989999771$

H $3.2715001106 \quad 6.0796999931-0.88580000401$

H $0.75720000267 \quad 7.5776000023-1.7998000383$

H $1.91540002826 .7678999901-2.8503000736$

H $1.3098000288 \quad 4.5541000366-1.9084999561$ 
$\mathrm{H}-0.11590000242 \quad 5.3793997765 \quad-2.4969000816$

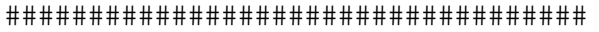

butylidene-eoctene

91

C $0.060600001365 \quad 0.31330001354 \quad 0.17679999769$

C $2.9590001106 \quad 0.24429999292-0.15710000694$

Ru $1.01510000231 .8774000406 \quad 0.028699999675$

Cl $1.02069997791 .6780999899-2.4272000790$

Cl $1.2446000576 \quad 2.2576999664 \quad 2.4609000683$

C $3.3731999397 \quad 1.4975999594 \quad 0.20999999344$

C $3.89389991762 .5457000732-0.74500000477$

C $5.42600011832 .6958999634-0.62690001726$

C $5.9727001190 \quad 3.7727999687-1.5690000057$

C $-0.59039998055-0.34099999070 \quad 1.3530000448$

C $-1.8760000467-1.11290001870 .98769998550$

C $-2.4978001118-1.8098000288 \quad 2.2021999359$

$\begin{array}{lllll}\text { C } 2.9045999050 & -0.91299998760 & 0.80790001154\end{array}$

C $4.2396998405-1.6878000498 \quad 0.82279998064$

C $4.1957001686-2.9007999897 \quad 1.7575000525$

H $0.013000000268-0.28380000591-0.74860000610$

H $2.8577001095 \quad 0.021099999547-1.2180000544$

H 3.55590009691 .67999994751 .2680000067

H $3.61139988902 .2943000793-1.7710000277$

H $3.4423000813 \quad 3.5239999294-0.52079999447$

H 5.69210004812 .93709993360 .41069999337

H $5.89440011981 .7290999889-0.85180002451$

H $7.0602998734 \quad 3.8642001152-1.4807000160$

H $5.73810005193 .5381000042-2.6131999493$

H $5.5355000496 \quad 4.7526001930-1.3436000347$

H $0.14929999411-1.0665999651 \quad 1.7351000309$

H $-0.74839997292 \quad 0.36860001087 \quad 2.1677999496$

$\mathrm{H}-2.6057999134-0.422699987890 .54739999771$

$\mathrm{H}-1.6459000111-1.8511999846 \quad 0.20829999447$

$\mathrm{H}-3.4094998837-2.3492999077 \quad 1.9253000021$

H $-1.8014999628-2.53099989892 .6451001167$

$\mathrm{H}-2.7607998848-1.08469998842 .9804999828$

H $2.6796000004-0.542800009251 .8150000572$

H $2.1066000462-1.61020004750 .52469998598$

H $4.4826002121-2.0139000416-0.19740000367$

H $5.0429000854-1.00629997251 .1303000450$

H $5.1508002281-3.4361000061 \quad 1.7597999573$

H $3.9790999889-2.59629988672 .7876999378$

H $3.4168000221-3.6082999706 \quad 1.4500000477$

P $-0.781400024893 .4684000015-0.093000002205$

C $-1.93060004713 .2567000389-1.5592000484$

C $-2.53139996531 .8427000046-1.6986999512$

C $-3.28859996801 .7062000036-3.0311000347$

C $-4.3727002144 \quad 2.7839000225-3.1851999760$

C $-3.7806000710 \quad 4.1922001839-3.0257999897$

C $-3.0239000320 \quad 4.3390002251-1.6928999424$

C $-1.7522000074 \quad 3.7109999657 \quad 1.5049999952$

C $-2.3498001099 \quad 5.1129999161 \quad 1.7660000324$

C $-2.9354999065 \quad 5.1922998428 \quad 3.1893999577$

C $-3.9832000732 \quad 4.0988001823 \quad 3.4439001083$

C $-3.4107000828 \quad 2.7060000896 \quad 3.1433999538$

C $-2.83710002902 .6338999271 \quad 1.7177000046$

C $0.017999999225 \quad 5.1491999626-0.38929998875$

C $1.0628000498 \quad 5.4720997810 \quad 0.70389997959$

C 1.64079999926 .88530015950 .52100002766

C $2.24469995507 .0686001778-0.87889999151$

C $1.2186000347 \quad 6.7318000793-1.9704999924$

C $0.63220000267 \quad 5.3186001778-1.7963999510$

$\mathrm{H}-1.2282999754 \quad 3.3594000340-2.3961000443$

$\mathrm{H}-1.7280999422 \quad 1.1037000418-1.6572999954$

H $-3.2221999168 \quad 1.6375999451-0.87139999866$

H $-2.5676000118 \quad 1.7892999649-3.8557999134$

$\mathrm{H}-3.7325999737 \quad 0.70560002327 \quad-3.1008999348$

H $-4.8705000877 \quad 2.6896998882-4.1579999924$

$\mathrm{H}-5.1479001045 \quad 2.6308999062 \quad-2.4196999073$

$\mathrm{H}-3.0868999958 \quad 4.3909997940 \quad-3.8548998833$ 
H $-4.5707001686 \quad 4.9510998726 \quad-3.0868999958$

$\mathrm{H}-3.7404000759 \quad 4.2449002266-0.86669999361$

$\mathrm{H}-2.5934998989 \quad 5.3446002007-1.6232999563$

$\mathrm{H}-0.96909999847 \quad 3.5313999653 \quad 2.2532999516$

$\mathrm{H}-1.5907000303 \quad 5.89260005951 .6564999819$

$\mathrm{H}-3.13940000535 .3329000473 \quad 1.0362000465$

$\mathrm{H}-2.1168000698 \quad 5.0854001045 \quad 3.9145998955$

$\mathrm{H}-3.3717999458 \quad 6.1859002113 \quad 3.3512001038$

$\mathrm{H}-4.3420000076 \quad 4.1501998901 \quad 4.4791002274$

$\mathrm{H}-4.8564000130 \quad 4.2768001556 \quad 2.7990999222$

$\mathrm{H}-2.6119999886 \quad 2.4741001129 \quad 3.8613998890$

$\mathrm{H}-4.18510007861 .9390000105 \quad 3.2706000805$

$\mathrm{H}-3.6544001102 \quad 2.7827000618 \quad 0.99989998341$

$\mathrm{H}-2.4296000004 \quad 1.6376999617 \quad 1.5326999426$

$\mathrm{H}-0.79979997873 \quad 5.8769998550 \quad-0.29879999161$

H $0.63550001383 \quad 5.3621997833 \quad 1.7051000595$

$\mathrm{H} \quad 1.8775000572 \quad 4.7390999794 \quad 0.65770000219$

H $0.84210002422 \quad 7.62639999390 .67309999466$

H 2.39520001417 .07560014721 .2940000296

H $2.61459994328 .0934000015-1.0055999756$

H $3.11509990696 .4050998688-0.98530000448$

H $0.40149998665 \quad 7.4675998688-1.9364000559$

$\mathrm{H} \quad 1.6742999554 \quad 6.8168001175-2.9644999504$

H $1.4098000526 \quad 4.5637001991-1.9598000050$

H $-0.121500000365 .1469001770-2.5710000992$

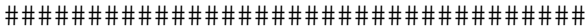

butylidene-ethylene-TS

73

C $-0.086599998176 \quad 0.051100000739-0.049499999732$

C $1.9071999788 \quad 0.0012000000570 \quad 0.099100001156$

Ru $1.38199996951 .8142999411-0.025900000706$

Cl $1.25890004631 .9203000069-2.4746000767$

Cl $1.2472000122 \quad 1.8662999868 \quad 2.4245998859$

C $-0.669099986551 .3739000559-0.012799999677$

C $2.4953999519-0.88569998741-0.96439999342$

C $2.2365999222-2.3877999783-0.73589998484$

C $2.9642000198-3.2593998909-1.7647000551$

H -1.10710000991 .71019995210 .92239999771$

$\mathrm{H}-1.0966999531 \quad 1.7538000345-0.93699997663$

$\mathrm{H}-0.20610000193-0.542900025840 .85290002823$

$\mathrm{H}-0.14589999616-0.47330000997-0.99809998274$

H $2.0374999046-0.371100008491 .1219999790$

H $2.1754999161-0.56760001183-1.9598000050$

H $3.5829000473-0.71359997988-0.91780000925$

H $2.5557999611-2.6630001068 \quad 0.27820000052$

H $1.1581000090-2.5854001045-0.78519999981$

H $2.7676000595-4.3227000237-1.5936000347$

H $2.6426000595-3.0176000595-2.7836999893$

H $4.0482997894-3.1064000130-1.7143000364$

P $3.0401000977 \quad 3.6070001125-0.0043999999762$

C $4.0549001694 \quad 3.60220003131 .5680999756$

C $4.8527002335 \quad 2.2969000340 \quad 1.7714999914$

$\begin{array}{llll}\text { C } 5.4387998581 & 2.2441999912 & 3.1926999092\end{array}$

C $6.3095002174 \quad 3.47440004353 .4914999008$

C $5.5356998444 \quad 4.77930021293 .2486000061$

C $4.9478998184 \quad 4.8341999054 \quad 1.8258999586$

C $4.08050012593 .6419000626-1.5598000288$

C $5.00939989094 .8603000641-1.7446000576$

C $5.6140999794 \quad 4.8614997864 \quad-3.1610999107$

C $6.3568000793 \quad 3.5497999191-3.4591999054$

C $5.45120000842 .3296000957-3.2306001186$

C $4.8466000557 \quad 2.3275001049-1.8159999847$

C $2.1451001167 \quad 5.25750017170 .0065000001341$

C $1.3456000090 \quad 5.5037999153-1.2928999662$

C $0.65579998493 \quad 6.8786997795 \quad-1.2628999949$

C $-0.24300000072 \quad 7.0387997627-0.028599999845$

C $0.54680001736 \quad 6.7834000587 \quad 1.2631000280$

C $1.2345000505 \quad 5.40789985661 .2460999489$

H $3.2560999393 \quad 3.58850002292 .3210999966$

H 4.20170021061 .43309998511 .6060999632 
H $5.67229986192 .2404000759 \quad 1.0437999964$

$\mathrm{H} \quad 4.6107001305 \quad 2.1926000118 \quad 3.9121999741$

H 6.02379989621 .32480001453 .3169999123

$\mathrm{H} 6.6777000427 \quad 3.4398000240 \quad 4.5240001678$

H $7.19670009613 .4567000866 \quad 2.8413999081$

H $4.7161002159 \quad 4.8576002121 \quad 3.9765999317$

H $6.1865000725 \quad 5.64720010763 .4119999409$

H $5.7712998390 \quad 4.8604998589 \quad 1.1016000509$

H $4.3845000267 \quad 5.7652997971 \quad 1.6987999678$

H $3.2941000462 \quad 3.6839001179-2.3245999813$

H $4.47149991995 .7994999886-1.5730999708$

H $5.8241000175 \quad 4.8235001564-1.0111000538$

H $4.8067998886 \quad 4.9980001450-3.8942999840$

$\mathrm{H} \quad 6.2898001671 \quad 5.7185997963-3.2730998993$

H $6.7365999222 \quad 3.5555999279-4.4879999161$

H $7.23500013353 .4770998955-2.8010001183$

$\mathrm{H} 4.63189983372 .3347001076-3.9621000290$

H $6.0141000748 \quad 1.4023000002-3.3933000565$

H $5.65269994742 .2107000351-1.0805000067$

$\mathrm{H} \quad 4.16790008541 .4776999950-1.7048000097$

H $2.9331998825 \quad 6.0213999748 \quad 0.068499997258$

H $1.9989999533 \quad 5.4425001144 \quad-2.1686999798$

$\mathrm{H} \quad 0.59619998932 \quad 4.7130999565-1.4206000566$

$\mathrm{H} 1.4226000309 \quad 7.6673002243-1.2575000525$

H $0.0746000036607 .0138998032-2.1828000546$

$\mathrm{H}-0.691699981698 .0392999649-0.010499999858$

$\mathrm{H}-1.07400000106 .3217000961-0.091600000858$

$\mathrm{H} 1.3085000515 \quad 7.5676999092 \quad 1.3840999603$

$\mathrm{H}-0.11219999939 \quad 6.8498001099 \quad 2.1368999481$

$\mathrm{H} \quad 0.47659999132 \quad 4.6147999763 \quad 1.2415000200$

H $1.8059999943 \quad 5.2677001953 \quad 2.1689000130$

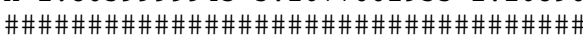

butylidene-pentene-12E-TS

82

C $-0.0346000008290 .012099999934-0.0048000002280$

C $1.9644999504-0.00130000000350 .052499998361$

$\mathrm{Ru} 1.44729995731 .81679999830 .011300000362$

Cl $1.24070000651 .9608999491 \quad-2.4333999157$

Cl $1.4200999737 \quad 1.8611999750 \quad 2.4612998962$

C $-0.58609998226 \quad 1.3598999977 \quad 0.038100000471$

C $-0.36430001259-0.91350001097 \quad 1.1633000374$

C $-1.8443000317-1.33720004561 .1375000477$

C $-2.1872000694-2.2994999886 \quad 2.2788000107$

C $2.5388000011-0.83289998770-1.0656000376$

C $2.3405001163-2.3517999649-0.90069997311$

C $3.0908000469-3.1454000473-1.9752999544$

$\mathrm{H}-1.0317000151 \quad 1.6815999746 \quad 0.97719997168$

$\mathrm{H}-1.02400004861 .7515000105-0.87580001354$

$\mathrm{H}-0.081000000238-0.43489998579-0.99769997597$

H $2.1454000473-0.403499990701 .0557999611$

H $0.25870001316-1.81389999391 .1282000542$

$\mathrm{H}-0.13359999657-0.39340001345 \quad 2.0989000797$

$\mathrm{H}-2.4779999256-0.44389998913 \quad 1.2015999556$

$\mathrm{H}-2.0741999149-1.8100999594 \quad 0.17270000279$

H $-3.2421998978-2.59069991112 .2497000694$

$\mathrm{H}-1.5861999989-3.2142999172 \quad 2.2195000648$

$\mathrm{H}-1.9925999641-1.8381999731 \quad 3.2534000874$

H $2.1726000309-0.48649999499-2.0350000858$

H $3.6210000515-0.62620002031-1.0539000034$

H $2.6803998947-2.6614999771 \quad 0.096799999475$

H $1.2704999447-2.5894999504-0.95240002871$

H $2.9368000031-4.2224998474-1.8539999723$

H $2.7500000000-2.8678998947-2.9788000584$

H $4.1686000824-2.9528000355-1.9266999960$

P $3.04550004013 .6635999680-0.011599999852$

C $4.1276001930 \quad 3.66659998891 .5139000416$

C $4.9342999458 \quad 2.3629000187 \quad 1.6915999651$

$\begin{array}{lllll}\text { C } 5.5728998184 & 2.3190000057 & 3.0901999474\end{array}$

C 6.45170021063 .55240011223 .3513000011

C $5.66919994354 .8558998108 \quad 3.1282000542$ 
C $5.0297999382 \quad 4.9001002312 \quad 1.7276999950$

C $4.01719999313 .7527999878-1.6078000069$

C $4.88219976435 .0118999481-1.8279000521$

C $5.4210000038 \quad 5.0426998138-3.2704000473$

C $6.2069997787 \quad 3.7660999298-3.6075000763$

C $5.36940002442 .5053999424-3.3413000107$

C $4.83090019232 .4753000736-1.9007999897$

C 2.09800004965 .28340005870 .062600001693

C $1.2252000570 \quad 5.5124998093-1.1923999786$

C $0.49300000072 \quad 6.8636999130-1.1175999641$

$\begin{array}{lllll}\text { C } & -0.34639999270 & 6.9864001274 & 0.16179999709\end{array}$

C $0.51690000296 \quad 6.75110006331 .4093999863$

C $1.24790000925 .3990001678 \quad 1.3482999802$

H $3.3624999523 \quad 3.65510010722 .3008999825$

$\mathrm{H} 4.2781000137 \quad 1.49820005891 .5565999746$

H $5.7255997658 \quad 2.3038001060 \quad 0.93300002813$

H 4.77120018012 .26920008663 .8392000198

H $6.1641998291 \quad 1.40129995353 .1981000900$

H $6.8555998802 \quad 3.5239999294 \quad 4.3706998825$

H 7.31589984893 .53169989592 .6707000732

$\mathrm{H} 4.8768000603 \quad 4.93940019613 .8852000237$

H $6.3257999420 \quad 5.7246999741 \quad 3.2616000175$

H $5.8263001442 \quad 4.9177999496 \quad 0.97359997034$

H 4.46330022815 .83099985121 .6122000217

H $3.1988000870 \quad 3.7618000507-2.3389999866$

H $4.31090021135 .9257001877-1.6298999786$

H $5.72970008855 .0113000870-1.1313999891$

H $4.57569980625 .1444001198-3.9653999805$

H $6.05229997635 .9291000366-3.4100999832$

H $6.5373001099 \quad 3.7906000614-4.6531000137$

H $7.1178998947 \quad 3.7323000431-2.9914000034$

H $4.51770019532 .4737000465-4.0341000557$

H $5.96649980551 .6052000523-3.5323998928$

H $5.6743998528 \quad 2.3970999718-1.2027000189$

H $4.19950008391 .5938999653-1.7597999573$

H $2.8633000851 \quad 6.07240009310 .089299999177$

$\mathrm{H} 1.8336999416 \quad 5.4772000313-2.1012001038$

H $0.49639999866 \quad 4.6989002228-1.2882000208$

H $1.2323999405 \quad 7.6778001785-1.1454999447$

$\mathrm{H}-0.13850000501 \quad 6.9847002029-2.0058999062$

$\mathrm{H}-0.82700002193 \quad 7.9713001251 \quad 0.20999999344$

$\mathrm{H}-1.1548000574 \quad 6.2417998314 \quad 0.13609999418$

H $1.2575999498 \quad 7.5599999428 \quad 1.4963999987$

$\mathrm{H}-0.098300002515 \quad 6.7915000916 \quad 2.3164000511$

$\mathrm{H} \quad 0.51829999685 \quad 4.5812001228 \quad 1.3799999952$

H $1.8704999685 \quad 5.2768998146 \quad 2.2400000095$

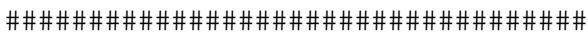

butylidene-pentene-12Z-TS

82

C $-0.032000001520-0.0083999997005-0.047800000757$

C $1.9671000242-0.068599998951-0.039500001818$

Ru $1.4847999811 \quad 1.7609000206-0.034299999475$

Cl $1.27400004862 .0683000088-2.4554998875$

Cl $1.4867999554 \quad 1.6339999437 \quad 2.4242000580$

C -0.542800009251 .35350000860 .10970000178$

C $-0.47850000858-0.77289998531-1.2855000496$

C $-1.9686000347-1.1532000303-1.1977000237$

C $-2.4300999641-1.9506000280-2.4217998981$

C $2.5989999771-0.83660000563-1.1729999781$

C $2.7774999142-2.3380999565-0.88059997559$

C $3.4934999943-3.0675001144-2.0216000080$

$\mathrm{H}-0.89840000868 \quad 1.64919996261 .0923999548$

$\mathrm{H}-1.0597000122 \quad 1.7779999971-0.74949997663$

$\mathrm{H}-0.057300001383-0.563600003720 .88999998569$

H $2.1338999271-0.50720000267 \quad 0.95020002127$

$\mathrm{H}-0.30039998889-0.15330000222-2.1703999043$

$\mathrm{H} 0.10279999673-1.6922999620-1.4057999849$

$\mathrm{H}-2.1449000835-1.7397999763-0.28549998999$

$\mathrm{H}-2.5720999241-0.24240000546-1.0994999409$

H $-3.4895999432-2.2163999081-2.3468000889$ 
$\mathrm{H}-2.2932999134-1.3715000153-3.3419001102$

$\mathrm{H}-1.8590999842-2.8801999092-2.5276999474$

H $2.0734999180-0.65770000219-2.1145999432$

H $3.5971999168-0.39640000463-1.3157999516$

H $3.3468999863-2.45499992370 .051100000739$

H $1.8004000187-2.8039000034-0.69969999790$

H $3.6257998943-4.1300997734-1.7940000296$

H $2.9261999130-2.9920001030-2.9560000896$

H $4.4853000641-2.6373999119-2.2025001049$

P $3.10710000993 .5871999264 \quad 0.076899997890$

C $4.2242999077 \quad 3.4375000000 \quad 1.5684000254$

$\begin{array}{llll}\text { C } 4.9819998741 & 2.0943000317 & 1.6345000267\end{array}$

C $5.6577000618 \quad 1.93289995193 .0069000721$

C 6.59070014953 .11240005493 .3234999180

C $5.85370016104 .4562001228 \quad 3.2121000290$

C $5.1799001694 \quad 4.6187000275 \quad 1.8368999958$

C $4.0440998077 \quad 3.8115999699-1.5291999578$

C $4.87230014805 .1047000885-1.6848000288$

C $5.39300012595 .2347002029-3.1285998821$

C $6.20790004734 .0022997856-3.5504999161$

C $5.40360021592 .7077000141-3.3559000492$

C $4.8836002350 \quad 2.5757999420-1.9143999815$

C $2.1816999912 \quad 5.2034001350 \quad 0.31220000982$

C $1.2148000002 \quad 5.4798998833-0.86220002174$

$\begin{array}{lllll}\text { C } & 0.49320000410 & 6.8256001472 & -0.68120002747\end{array}$

C $-0.24619999528 \quad 6.8948998451 \quad 0.66250002384$

C $0.70999997854 \quad 6.6132001877 \quad 1.8302999735$

C $1.4354000092 \quad 5.2666997910 \quad 1.6635999680$

H $3.4791998863 \quad 3.3993000984 \quad 2.3733000755$

H $4.28550004961 .2683000565 \quad 1.4650000334$

H $5.7476000786 \quad 2.0539999008 \quad 0.84890002012$

H $4.8761000633 \quad 1.8618999720 \quad 3.7751998901$

H $6.21530008320 .98869997263 \quad 3.0353000164$

H $7.02120018012 .9995999336 \quad 4.3257999420$

H $7.43410015113 .1061000824 \quad 2.6171998978$

H $5.0854001045 \quad 4.51690006263 .9956998825$

H $6.54680013665 .2890000343 \quad 3.3845999241$

H $5.9567999840 \quad 4.65390014651 .0633000135$

$\mathrm{H} 4.64839982995 .57639980321 .7960000038$

H $3.2119998932 \quad 3.8464999199-2.2439999580$

H $4.27729988105 .9888000488-1.4305000305$

H $5.72709989555 .0901999474-0.99800002575$

H $4.53690004355 .3545999527-3.8073000908$

H $5.99870014196 .1447000504 \quad-3.2212998867$

H $6.5275001526 \quad 4.0996999741-4.5952000618$

H $7.1248998642 \quad 3.9532999992 \quad-2.9449000359$

H $4.54510021212 .6984000206-4.0408000946$

H $6.01919984821 .8353999853-3.6080000401$

H $5.73740005492 .4732999802-1.2319999933$

H $4.2744998932 \quad 1.6720000505-1.8222999573$

H $2.9505000114 \quad 5.9892997742 \quad 0.31250000000$

H $1.74829995635 .4710001945-1.8177000284$

$\mathrm{H} \quad 0.47589999437 \quad 4.6715002060-0.92530000210$

$\mathrm{H} 1.2294000387 \quad 7.6412000656-0.73350000381$

$\mathrm{H}-0.20399999619 \quad 6.9794998169 \quad-1.5135999918$

$\begin{array}{lllll}\mathrm{H} & -0.72229999304 & 7.8751001358 & 0.78659999371\end{array}$

$\mathrm{H}-1.0536999702 \quad 6.1487998962 \quad 0.66879999638$

H $1.4543999434 \quad 7.42080020901 .8913999796$

H $0.16590000689 \quad 6.6167001724 \quad 2.7822999954$

H $0.71499997377 \quad 4.4425001144 \quad 1.7250000238$

H $2.1298999786 \quad 5.1205000877 \quad 2.4967000484$

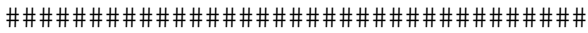

butylidene-pentene-21TS

82

C $-0.0245999991890 .023700000718-0.013299999759$

C $2.1751000881-0.014399999753-0.021199999377$

$\mathrm{Ru} 0.65890002251 \quad 1.7618000507-0.0031000000890$

Cl $0.69510000944 \quad 1.6861000061-2.4625000954$

Cl $0.76959997416 \quad 1.9921000004 \quad 2.4514000416$

C $2.79200005531 .2402000427 \quad 0.18330000341$ 
C $3.64019989971 .9377000332-0.85600000620$

C $5.1409997940 \quad 1.6571999788-0.63220000267$

C $6.0220999718 \quad 2.4052999020-1.6373000145$

C $-0.57029998302-0.81129997969 \quad 1.1062999964$

C $-0.42449998856-2.3303999901 \quad 0.89689999819$

C $-1.1353000402-3.13210010531 .9922000170$

H $-0.16099999845-0.41589999199-1.0112999678$

H $2.0850000381-0.678499996660 .83230000734$

H $2.2223000526-0.46840000153-1.0070999861$

H $3.01530003551 .4943000078 \quad 1.2180999517$

H $3.33540010451 .6359000206-1.8609999418$

H $3.47959995273 .0234000683-0.79250001907$

H $5.4191999435 \quad 1.9429999590 \quad 0.39109998941$

H $5.3186998367 \quad 0.57649999857-0.71079999208$

H $7.08370018012 .1946001053-1.4709000587$

H $5.77869987492 .1166999340-2.6659998894$

H $5.87909984593 .4890000820-1.5555000305$

H $-0.17049999535-0.486099988222 .0704998970$

$\mathrm{H}-1.6468000412-0.57020002604 \quad 1.1312999725$

$\mathrm{H}-0.82880002260-2.6031000614-0.087099999189$

$\mathrm{H} 0.63980001211-2.59870004650 .87809997797$

$\mathrm{H}-1.0197000504-4.2093000412 \quad 1.8350000381$

$\mathrm{H}-0.73180001974 \quad-2.89120006562 .9818999767$

$\mathrm{H}-2.2083001137-2.9087998867 \quad 2.0092000961$

$\begin{array}{lllll}\mathrm{P} & -1.0844999552 & 3.4684000015 & -0.083499997854\end{array}$

C $-2.22300004963 .2762000561-1.5578999519$

C $-2.8694000244 \quad 1.8797999620-1.6769000292$

C $-3.5769999027 \quad 1.7324999571-3.0353999138$

C $-4.6171998978 \quad 2.8413999081 \quad-3.2597999573$

C $-3.99079990394 .2353000641-3.1005001068$

C $-3.28360009194 .3828001022-1.7405999899$

C $-2.0257000923 \quad 3.6735999584 \quad 1.5278999805$

C $-2.8357000351 \quad 4.9752001762 \quad 1.7180999517$

C $-3.3680000305 \quad 5.07210016253 .1607999802$

C $-4.2052998543 \quad 3.8420999050 \quad 3.5425999165$

C $-3.41669988632 .5425999165 \quad 3.3196001053$

C $-2.8880999088 \quad 2.4430999756 \quad 1.8782999516$

C $-0.25929999352 \quad 5.1395001411-0.33849999309$

$\begin{array}{lllll}\text { C } & 0.75389999151 & 5.4369997978 & 0.79089999199\end{array}$

C $1.3792999983 \quad 6.8320999146 \quad 0.62459999323$

$\begin{array}{lllll}\text { C } 2.0357000828 & 6.9955000877 & -0.75389999151\end{array}$

C $1.0375000238 \quad 6.6873998642-1.8795000315$

C $0.408100008965 .2909002304-1.7231999636$

$\mathrm{H}-1.5008000135 \quad 3.3396000862-2.3819000721$

$\mathrm{H}-2.10109996801 .1086000204-1.5753999949$

$\mathrm{H}-3.6008000374 \quad 1.7302000523-0.87250000238$

$\mathrm{H}-2.82010006901 .7699999809-3.8306999207$

$\mathrm{H}-4.05319976810 .74610000849-3.0991001129$

$\mathrm{H}-5.0729999542 \quad 2.7421000004 \quad-4.2526998520$

$\mathrm{H}-5.4310998917 \quad 2.7283000946-2.5283000469$

H $-3.25970005994 .4008998871-3.9044001102$

$\mathrm{H}-4.7564997673 \quad 5.0142998695 \quad-3.2053000927$

$\mathrm{H}-4.0336999893 \quad 4.3112998009-0.94279998541$

$\mathrm{H}-2.8347001076 \quad 5.3796000481-1.6635999680$

$\mathrm{H}-1.1971000433 \quad 3.6777999401 \quad 2.2476999760$

$\mathrm{H}-2.2235000134 \quad 5.8571000099 \quad 1.5023000240$

$\mathrm{H}-3.6835000515 \quad 5.0009999275 \quad 1.0226999521$

H $-2.5165998936 \quad 5.1595997810 \quad 3.8503999710$

$\mathrm{H}-3.9600999355 \quad 5.9890999794 \quad 3.2734999657$

$\mathrm{H}-4.5329999924 \quad 3.9159998894 \quad 4.5869002342$

H $-5.1177000999 \quad 3.8224999905 \quad 2.9279999733$

$\mathrm{H}-2.56380009652 .5048000813 \quad 4.0106000900$

$\mathrm{H}-4.04570007321 .6720999479 \quad 3.5445001125$

$\mathrm{H}-3.74070000652 .3747999668 \quad 1.1898000240$

$\mathrm{H}-2.2948999405 \quad 1.5314999819 \quad 1.7635999918$

$\mathrm{H}-1.0650000572 \quad 5.8839001656-0.27439999580$

H $0.28130000830 \quad 5.3544001579 \quad 1.7746000290$

H $1.5473999977 \quad 4.6778998375 \quad 0.77859997749$

H $0.59659999609 \quad 7.5953001976 \quad 0.74760001898$

H $2.1112999916 \quad 7.00339984891 .4232000113$

H $2.43880009658 .0094003677-0.86760002375$ 
H $2.8896000385 \quad 6.3067998886-0.83050000668$

H $0.240500003107 .4458999634-1.8732999563$

H $1.52849996096 .7582998276-2.8577001095$

$\mathrm{H} 1.1721999645 \quad 4.5160999298-1.8554999828$

H $-0.32129999995 \quad 5.1321997643-2.5236999989$

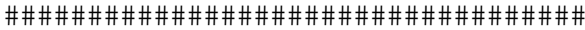

butylidene-pentene

82

C $-0.296299993990 .39700001478 \quad-0.0024999999441$

C $2.5690000057 \quad 0.416399985550 .24639999866$

$\mathrm{Ru} 1.70000004772 .0234999657 \quad 0.045099999756$

Cl $1.5784000158 \quad 1.9847999811 \quad-2.4119999409$

$\begin{array}{llllll}\text { Cl } & 1.4631999731 & 2.2718000412 & 2.4779999256\end{array}$

C $-0.63249999285 \quad 1.7228000164 \quad 0.040699999779$

C $-0.39370000362-0.52259999514 \quad 1.1855000257$

C $-1.7770999670-1.2070000172 \quad 1.2431000471$

C $-1.8904000521-2.1761999130 \quad 2.4237000942$

C $3.3661999702-0.38190001249-0.73549997807$

C $2.8041000366-1.8105000257-0.92250001431$

C $3.6742999554-2.6452000141-1.8680000305$

$\mathrm{H}-0.938000023372 .1807000637 \quad 0.97750002146$

$\mathrm{H}-0.792299985892 .2709000111-0.88429999352$

$\mathrm{H}-0.12120000273-0.049199998379-0.97970002890$

H $2.4539000988-0.067500002682 \quad 1.2288000584$

H $0.37670001388-1.30050003531 .1273000240$

$\mathrm{H}-0.21510000527 \quad 0.0489000007512 .1024000645$

$\mathrm{H}-2.5538001060-0.43549999595 \quad 1.3158999681$

$\mathrm{H}-1.9613000154-1.7440999746 \quad 0.30289998651$

$\mathrm{H}-2.8761000633-2.6517999172 \quad 2.4542999268$

H $-1.1375999451-2.9700999260 \quad 2.3563001156$

$\mathrm{H}-1.7381000519-1.6549999714 \quad 3.3752000332$

H $3.43070006370 .12590000033-1.6996999979$

H $4.3846998215-0.47220000625-0.32019999623$

H $2.7277998924-2.3080999851 \quad 0.053300000727$

H $1.7869000435-1.7404999733-1.3252999783$

H $3.2616999149-3.6507000923-2.0000998974$

H $3.7393999100-2.1765999794-2.8559000492$

H $4.6939001083-2.7488000393-1.4793000221$

P 3.56240010263 .52239990230 .046000000089

C $4.63240003593 .4512999058 \quad 1.5815000534$

C $5.1610999107 \quad 2.0429999828 \quad 1.9253000021$

C $5.8589000702 \quad 2.0457000732 \quad 3.2964999676$

C $6.9835000038 \quad 3.0901999474 \quad 3.3624000549$

C 6.45989990234 .48969984053 .0069000721

C $5.7639999390 \quad 4.50050020221 .6332999468$

C $4.60500001913 .5192999840-1.5262000561$

C $5.2343001366 \quad 4.8653998375-1.9527000189$

C $5.90399980554 .7389998436-3.3348000050$

C $6.9478998184 \quad 3.6138000488-3.3703999519$

C $6.33419990542 .2813000679-2.9173998833$

C $5.67969989782 .4098999500-1.5310000181$

C $2.8022999763 \quad 5.2456998825 \quad 0.093299999833$

C $1.74199998385 .4000000954-1.0220999718$

C $1.1977000237 \quad 6.8366999626-1.0737999678$

C 0.620500028137 .26539993290 .28330001235

C 1.65450000767 .08699989321 .4048999548

C $2.2060999870 \quad 5.6501002312 \quad 1.4595999718$

H $3.8966999054 \quad 3.6877999306 \quad 2.3610000610$

H 4.32520008091 .33910000321 .9421000481

$\mathrm{H} 5.87069988251 .70210003851 .1617000103$

H $5.1119999886 \quad 2.2616000175 \quad 4.0727000237$

H $6.2543001175 \quad 1.04499995713 .5100998878$

H $7.4398999214 \quad 3.0978999138 \quad 4.3596000671$

$\begin{array}{lllll}\text { H } & 7.7789001465 & 2.8127000332 & 2.6549999714\end{array}$

H $5.7430000305 \quad 4.81479978563 .7739000320$

H 7.27890014655 .21969985963 .0113000870

H $6.5081000328 \quad 4.2821002007 \quad 0.85650002956$

H $5.3766999245 \quad 5.5043997765 \quad 1.4241000414$

H $3.8527998924 \quad 3.2434999943-2.2771999836$

H $4.47760009775 .6536998749-2.0030000210$ 
H $5.98120021825 .1873002052-1.2151000500$

H $5.1277999878 \quad 4.5362000465-4.0858998299$

H $6.36299991615 .6974000931-3.6077001095$

H $7.3698000908 \quad 3.5183999538-4.3782000542$

H $7.7831001282 \quad 3.8726000786-2.7030000687$

H $5.5739998817 \quad 1.9610999823-3.6431000233$

H $7.09980010991 .4958000183-2.8926999569$

H $6.4587001801 \quad 2.6443998814 \quad-0.79409998655$

H $5.2432999611 \quad 1.4519000053-1.2397999763$

H $3.6270999908 \quad 5.9376997948-0.12250000238$

H $2.14140009885 .1036000252-1.9969999790$

H $0.90960001945 \quad 4.7093000412-0.82609999180$

H $2.0118000507 \quad 7.5201001167-1.3572000265$

H $0.43610000610 \quad 6.9141001701-1.8590999842$

H $0.27840000391 \quad 8.3066997528 \quad 0.24189999700$

H $-0.263700008396 .6515998840 \quad 0.50760000944$

H $2.4872000217 \quad 7.7876000404 \quad 1.2430000305$

H $1.2139999866 \quad 7.3442997932 \quad 2.3756999969$

H 1.41499996194 .94810009001 .7457000017

H $2.9647998810 \quad 5.5892000198 \quad 2.2462999821$

\#\#\#\#\#\#\#\#\#\#\#\#\#\#\#\#\#\#\#\#\#\#\#\#\#\#\#\#\#\#\#\#\#\#\#

butylidene-zoctene-E-TS

91

C $-0.034000001848 \quad 0.0073000001721-0.0017000000225$

C $1.9646999836-0.064900003374 \quad 0.0041999998502$

$\mathrm{Ru} 1.5010999441 \quad 1.7720999718 \quad 0.0063999998383$

Cl $1.42949998381 .9779000282 \quad-2.4595999718$

Cl $1.5118000507 \quad 1.7583999634 \quad 2.4637000561$

C $-0.54750001431 \quad 1.3693000078-0.21410000324$

C $2.5713000298-0.83410000801-1.1432000399$

C $2.6003999710-2.3645000458-0.98699998856$

C $3.4288001060-3.0327999592-2.0897998810$

C $-1.4582999945 \quad 2.0745999813 \quad 0.77090001106$

C $-2.9479000568 \quad 1.8654999733 \quad 0.42429998517$

C $-3.8761000633 \quad 2.6217999458 \quad 1.3798999786$

C $-0.48109999299-0.749700009821 .2486000061$

C $-0.20630000532-2.2579998970 \quad 1.2431999445$

C $-0.83859997988-2.95600008962 .4521000385$

$\mathrm{H}-0.772300004961 .5968999863-1.2551000118$

$\mathrm{H}-0.048900000751-0.56989997625-0.92729997635$

H $2.1389999390-0.500500023370 .99459999800$

H $2.1154999733-0.53609997034-2.0906999111$

H $3.6124000549-0.47799998522-1.1992000341$

H $3.0139999390-2.6277000904-0.0038999998942$

$\mathrm{H} \quad 1.5779000521-2.7588000298-1.0085999966$

H $3.4346001148-4.1220002174-1.9788000584$

H $3.0262000561-2.7981998920-3.0815999508$

H $4.4689002037-2.6875998974-2.0659000874$

$\mathrm{H}-1.2479000092 \quad 1.7664999962 \quad 1.7971999645$

H $-1.2515000105 \quad 3.1531000137 \quad 0.73009997606$

$\mathrm{H}-3.1270999908 \quad 2.1953999996-0.60759997368$

$\mathrm{H}-3.1854999065 \quad 0.79379999638 \quad 0.44819998741$

$\mathrm{H}-4.92850017552 .4709999561 \quad 1.1174000502$

$\mathrm{H}-3.7362999916 \quad 2.2869999409 \quad 2.4138998985$

$\mathrm{H}-3.6751000881 \quad 3.6989998817 \quad 1.3518999815$

$\mathrm{H}-0.035300001502-0.286599993712 .1342000961$

H $-1.5664000511-0.606000006201 .3403999805$

H $-0.59579998255-2.70289993290 .31650000811$

H $0.87290000916-2.44449996951 .2453000546$

$\mathrm{H}-0.63349997997-4.0314998627 \quad 2.4433999062$

$\mathrm{H}-0.44600000978-2.5471000671 \quad 3.3898000717$

H $-1.9264999628 \quad-2.8227000237 \quad 2.4630999565$

P $3.1568000317 \quad 3.57399988170 .059700001031$

C $4.3292999268 \quad 3.3861999512 \quad 1.5041999817$

C $4.98519992831 .9917000532 \quad 1.5952999592$

C 5.72970008851 .84220004082 .9331998825

C $6.7676000595 \quad 2.9572000504 \quad 3.1368000507$

C $6.1284999847 \quad 4.3481998444 \quad 3.0035998821$

C $5.3845000267 \quad 4.50050020221 .6640000343$

C $4.0571999550 \quad 3.8282999992-1.5655000210$ 
C $4.7930002213 \quad 5.1705999374-1.7700999975$

C $5.29449987415 .2871999741-3.2223000526$

C $6.1869997978 \quad 4.1002998352-3.6154999733$

C $5.4725999832 \quad 2.7618000507-3.3736000061$

C $4.9762001038 \quad 2.6414000988-1.9228999615$

C $2.2644999027 \quad 5.2023000717 \quad 0.35080000758$

C $1.18649995335 .4300999641-0.73470002413$

C $0.48649999499 \quad 6.7867999077-0.55169999599$

C $-0.11360000074 \quad 6.9264001846 \quad 0.85479998589$

C $0.95420002937 \quad 6.69490003591 .9336999655$

C $1.6567000151 \quad 5.3352999687 \quad 1.7641999722$

H $3.6287999153 \quad 3.43950009352 .3468999863$

$\mathrm{H} \quad 4.21659994131 .21899998191 .5121999979$

H $5.6932001114 \quad 1.8502000570 \quad 0.76880002022$

H $4.99340009691 .8696999550 \quad 3.7479999065$

H $6.2137999535 \quad 0.858900010592 .9788999557$

H 7.25029993062 .85520005234 .1163997650

H $7.56209993362 .8538999557 \quad 2.3829998970$

H $5.4173002243 \quad 4.5036997795 \quad 3.8271000385$

H $6.8916001320 \quad 5.1314001083 \quad 3.0938999653$

H $6.1126999855 \quad 4.44040012360 .84530001879$

H $4.9236001968 \quad 5.4935998917 \quad 1.6057000160$

H $3.2195000648 \quad 3.7878000736-2.2727000713$

H $4.1360001564 \quad 6.0187001228-1.5499999523$

H $5.64830017095 .2470998764-1.0879000425$

H $4.4268999100 \quad 5.3274998665-3.8958001137$

H $5.83589982996 .2329998016-3.3492000103$

H $6.4899001122 \quad 4.1873002052-4.6661000252$

H $7.1107001305 \quad 4.1311001778-3.0186998844$

H $4.60960006712 .6751999855-4.0471000671$

H $6.14230012891 .9247000217-3.6068000793$

H $5.84210014342 .6224999428-1.2476999760$

H $4.43359994891 .7000999451-1.7940000296$

H 3.02850008015 .98470020290 .24189999700

H $1.6201000214 \quad 5.3640999794 \quad-1.7375999689$

H $0.44119998813 \quad 4.6252999306-0.67900002003$

$\mathrm{H} \quad 1.2144999504 \quad 7.5943999290-0.71840000153$

H $-0.290899991996 .9036998749-1.3164000511$

$\mathrm{H}-0.573400020607 .91459989550 .97790002823$

H $-0.91799998283 \quad 6.1870999336 \quad 0.97890001535$

$\mathrm{H} 1.7026000023 \quad 7.49940013891 .8792999983$

H $0.50739997625 \quad 6.7505998611 \quad 2.9337000847$

$\mathrm{H} \quad 0.94840002060 \quad 4.5187997818 \quad 1.9437999725$

H $2.4340000153 \quad 5.23409986502 .5283000469$

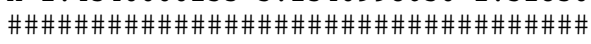

butylidene-zoctene-Z-TS

91

C $-0.087999999523 \quad 0.067299999297 \quad-0.037799999118$

C $1.9039000273-0.016200000420 \quad 0.12099999934$

$\mathrm{Ru} 1.4438999891 \quad 1.8187999725 \quad 0.016499999911$

Cl $1.49660003191 .9588999748 \quad-2.4328000546$

Cl $1.3389999866 \quad 1.7829999924 \quad 2.4876000881$

C $-0.60350000858 \quad 1.4345999956 \quad 0.16159999371$

C $2.6547999382-0.81730002165-0.92070001364$

C $3.3749001026-2.0374000072-0.32190001011$

C $4.1683001518-2.8183999062-1.3737000227$

C $-1.49360001092 .1307001114-0.84920001030$

C $-2.9879000187 \quad 1.8789000511-0.55419999361$

C $-3.9054999352 \quad 2.6033000946-1.5444999933$

C $-0.49419999123-0.72130000591-1.2789000273$

C $-0.49450001121-2.2400000095-1.0487999916$

C $-0.91449999809-3.0202000141-2.2985000610$

$\mathrm{H}-0.87150001526 \quad 1.6507999897 \quad 1.1949000359$

$\mathrm{H}-0.14720000327-0.49439999461 \quad 0.89480000734$

H $1.9845000505-0.416399985551 .1363999844$

H $1.9922000170-1.1191999912-1.7386000156$

H $3.3854000568-0.15909999609-1.4040999413$

H $4.0499000549-1.69920003410 .47560000420$

H $2.6428999901-2.6993000507 \quad 0.15989999473$

H $4.6880002022-3.6728000641-0.92809998989$ 
H $3.5099000931-3.2000000477-2.1624000072$

H $4.9205999374-2.1812000275-1.8526999950$

$\mathrm{H}-1.3157999516 \quad 3.2133998871-0.79290002584$

$\mathrm{H}-1.2387000322 \quad 1.8377000093-1.8701000214$

$\mathrm{H}-3.1933999062 \quad 0.80059999228 \quad-0.58069998026$

$\mathrm{H}-3.2126998901 \quad 2.2060000896 \quad 0.46959999204$

$\mathrm{H}-4.9612998962 \quad 2.4242999554-1.3150000572$

$\mathrm{H}-3.7362000942 \quad 3.6860001087-1.5181000233$

$\mathrm{H}-3.7228000164 \quad 2.2660000324-2.5710000992$

$\mathrm{H}-1.5104999542-0.42120000720-1.5615999699$

H $0.13500000536-0.44459998608-2.1315000057$

H $0.49829998612-2.5741000175-0.72460001707$

$\mathrm{H}-1.1772999763-2.4744999409-0.22050000727$

$\mathrm{H}-0.92890000343-4.0987000465-2.1096999645$

$\mathrm{H}-1.9164999723-2.7256999016-2.6310999393$

H $-0.22419999540-2.8343999386-3.1294000149$

P $3.0151000023 \quad 3.69650006290 .16410000622$

C 3.95180010803 .75130009651 .7833000422

C $4.78929996492 .4793999195 \quad 2.0367999077$

C $5.3168997765 \quad 2.4656000137 \quad 3.4814999104$

C 6.11920022963 .73539996153 .8064999580

C $5.3008999825 \quad 5.00339984893 .5172998905$

C $4.78020000465 .0215001106 \quad 2.0678999424$

C $4.1380000114 \quad 3.8157000542-1.3296999931$

C $4.9780001640 \quad 5.1023998260-1.4751000404$

C $5.6483998299 \quad 5.1466999054-2.8615999222$

C $6.5026998520 \quad 3.8949000835-3.1175999641$

C $5.68610000612 .6078999043-2.9209001064$

C $5.0213999748 \quad 2.5678000450-1.5346000195$

C $2.0181999207 \quad 5.2895998955 \quad 0.13349999487$

C $1.3121000528 \quad 5.5204000473-1.2220000029$

C $0.51990002394 \quad 6.8393998146-1.2175999880$

C $-0.48460000753 \quad 6.8980998993-0.057999998331$

$\begin{array}{lllll}\text { C } & 0.21719999611 & 6.6683998108 & 1.2881000042\end{array}$

C $1.0047999620 \quad 5.3474998474 \quad 1.2997000217$

H 3.12190008163 .71070003512 .5000000000

H $4.17560005191 .5920000076 \quad 1.8575999737$

H $5.64029979712 .4465999603 \quad 1.3442000151$

$\mathrm{H} 4.46140003202 .3822999001 \quad 4.1651000977$

H $5.9356999397 \quad 1.5737999678 \quad 3.6396000385$

H $6.4429998398 \quad 3.7248001099 \quad 4.8543000221$

$\mathrm{H} 7.03389978413 .75219988823 .1956000328$

H $4.4450998306 \quad 5.0497999191 \quad 4.2052998543$

H $5.9043998718 \quad 5.90059995653 .7035999298$

H $5.6366000175 \quad 5.0736999512 \quad 1.3846000433$

$\mathrm{H} 4.18680000315 .92780017851 .9023000002$

H $3.39540004733 .7943000793-2.1377000809$

H $4.36250019075 .9979000092-1.3343000412$

H $5.75649976735 .1297001839-0.70310002565$

H $4.8694000244 \quad 5.2202000618-3.6333999634$

H $6.26200008396 .0521998405-2.9460000992$

H $6.9267997742 \quad 3.9268999100-4.1284999847$

H $7.3534002304 \quad 3.8926000595-2.4203000069$

H $4.90170001982 .5448999405-3.6872000694$

$\mathrm{H} 6.32730007171 .7273000479-3.0508000851$

H $5.79969978332 .5253000259-0.76209998131$

H $4.41499996191 .6631000042-1.4392999411$

H $2.7469000816 \quad 6.1002998352 \quad 0.27660000324$

H $2.03949999815 .5352001190-2.0395998955$

H $0.63940000534 \quad 4.6816000938-1.4363000393$

$\mathrm{H} \quad 1.2210999727 \quad 7.6824998856-1.1313999891$

H $0.00549999997026 .9573998451-2.1789999008$

$\mathrm{H}-1.0105999708 \quad 7.8604998589-0.056299999356$

$\mathrm{H}-1.2497999668 \quad 6.1219000816 \quad-0.20329999924$

$\begin{array}{llllll}\mathrm{H} & 0.90609997511 & 7.5030999184 & 1.4848999977\end{array}$

$\mathrm{H}-0.51200002432 \quad 6.6648998260 \quad 2.1071000099$

H $0.30919998884 \quad 4.5029997826 \quad 1.2202999592$

H $1.5113999844 \quad 5.2246999741 \quad 2.2620000839$

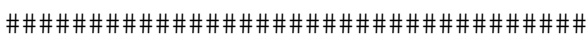

butylidene-zoctene

91 
$\begin{array}{lllll}\text { C } & -0.27669999003 & 0.32859998941 & 0.026499999687\end{array}$ $\begin{array}{lllll}\text { C } 2.7295999527 & 0.29789999127 & 0.020400000736\end{array}$ $\mathrm{Ru} 1.82299995421 .8996000290-0.0059000002220$ Cl $1.6756999493 \quad 2.0792000294 \quad-2.4647998810$ Cl $1.7246999741 \quad 1.8819999695 \quad 2.4546000957$ C $-0.59969997406 \quad 1.6503000259-0.14759999514$ C $3.4400999546-0.44319999218-1.0686000586$ C $3.0083000660-1.9221999645-1.1878999472$ C $3.8268001080-2.6693000793-2.2465000153$ C $-1.2783999443 \quad 2.5211999416 \quad 0.88220000267$ C -2.79920005802 .60240006450 .62400001287$ C $-3.5111000538 \quad 3.5081000328 \quad 1.6332999468$

C $-0.51759999990-0.46029999852 \quad 1.2890000343$ C $0.017599999905-1.8960000277 \quad 1.2482000589$ C $-0.31600001454-2.6824998856 \quad 2.5190000534$ H $-0.59289997816 \quad 2.0244998932-1.1700999737$ H $0.011500000022-0.22519999743-0.86500000954$ H $2.7511000633-0.213300004600 .99500000477$ H $3.3348000050 \quad 0.065499998629-2.0288000107$ H $4.5093002319-0.43309998512-0.79329997301$ H $3.1150999069-2.4175999165-0.21410000324$

H $1.9438999891-1.9661999941-1.4472999573$ H $3.5106000900-3.7147998810-2.3250999451$ H $3.7091000080-2.2068998814-3.2327001095$ H $4.8949999809-2.6596999168-2.0000000000$ $\mathrm{H}-1.08060002332 .15599989891 .8927999735$ $\mathrm{H}-0.86549997330 \quad 3.5388000011 \quad 0.83649998903$

$\mathrm{H}-2.97630000112 .9681999683-0.39610001445$

$\mathrm{H}-3.22420001031 .59099996090 .66540002823$

$\mathrm{H}-4.5875000954 \quad 3.5538001060 \quad 1.4377000332$

$\mathrm{H}-3.37030005463 .14420008662 .6572999954$

$\mathrm{H}-3.1189000607 \quad 4.5307998657 \quad 1.5894999504$

$\mathrm{H}-0.089299999177 \quad 0.075199998915 \quad 2.1435999870$

$\mathrm{H}-1.6058000326-0.49219998717 \quad 1.4582999945$

$\mathrm{H}-0.38890001178-2.4182999134 \quad 0.37099999189$

$\mathrm{H} 1.1051000357-1.87119996551 .1145000458$

H $0.079499997199-3.70239996912 .4725000858$

H $0.11190000176-2.1967999935 \quad 3.4028999805$

$\mathrm{H}-1.3995000124-2.7509000301 \quad 2.6707999706$

P $3.64299988753 .4416999817 \quad 0.090400002897$

$\begin{array}{lllll}\text { C } 4.7722997665 & 3.2614998817 & 1.5731999874\end{array}$

C 5.35699987411 .84640002251 .7596999407

C $6.09840011601 .7411999702 \quad 3.1038000584$

C 7.19129991532 .81200003623 .2390999794

C 6.61509990694 .22039985663 .0311000347

C 5.87569999694 .33500003811 .6854000092

C $4.6224999428 \quad 3.6242001057-1.5073000193$

C $5.2664999962 \quad 4.9995999336-1.7943999767$

C $5.8590002060 \quad 5.0307998657-3.2165999413$

C $6.8656997681 \quad 3.8942999840-3.4472000599$

C $6.23880004882 .5295999050-3.1275999546$

C $5.6610999107 \quad 2.4995000362-1.7026000023$

$\begin{array}{llll}\text { C } 2.8375999928 & 5.1307001114 & 0.31740000844\end{array}$

C $1.77040004735 .3716998100-0.77640002966$

C $1.1747000217 \quad 6.7852997780-0.67250001431$

C $0.59380000830 \quad 7.0483999252 \quad 0.72439998388$

$\begin{array}{llll}\text { C } 1.6442999840 & 6.7940001488 & 1.8154000044\end{array}$

C $2.2414000034 \quad 5.3773999214 \quad 1.7206000090$

$\mathrm{H} \quad 4.0620999336 \quad 3.3993999958 \quad 2.3982999325$

H 4.54720020291 .11440002921 .7317999601

H $6.05210018161 .6087000370 \quad 0.94520002604$

H $5.36959981921 .8554999828 \quad 3.9177999496$

H $6.5317997932 \quad 0.73890000582 \quad 3.2079000473$

H $7.6760997772 \quad 2.7413001060 \quad 4.2203001976$

H 7.97380018232 .62870001792 .4881000519

H 5.91319990164 .44999980933 .8452000618

H $7.4117999077 \quad 4.9731001854 \quad 3.0799999237$

H $6.5996999741 \quad 4.2073001862 \quad 0.87040001154$

H $5.4559998512 \quad 5.3422999382 \quad 1.5794999599$

H $3.83439993863 .4577000141-2.2532999516$ 
H $4.5309000015 \quad 5.8049001694-1.7037999630$

H $6.0601000786 \quad 5.2102999687-1.0663000345$

H $5.0390000343 \quad 4.9404997826-3.9428999424$

H $6.3316998482 \quad 6.0047001839-3.3949000835$

H $7.2312002182 \quad 3.9152998924-4.4809999466$

H $7.7417998314 \quad 4.0499000549-2.8004999161$

H $5.43190002442 .3183999062-3.8422999382$

H $6.9819002151 \quad 1.7308000326-3.2427000999$

H $6.48180007932 .6205999851-0.98339998722$

H $5.2059998512 \quad 1.5257999897-1.5113999844$

H $3.6435999870 \quad 5.8621001244 \quad 0.16899999976$

H $2.18140006075 .2024002075-1.7766000032$

$\mathrm{H} 0.963400006294 .6338000298-0.66500002146$

H $1.9610999823 \quad 7.5243000984-0.88529998064$

H $0.40490001440 \quad 6.9163999557 \quad-1.4427000284$

H $0.21150000393 \quad 8.0740995407 \quad 0.79290002584$

$\mathrm{H}-0.26489999890 \quad 6.3812999725 \quad 0.88880002499$

H $2.4512999058 \quad 7.5353999138 \quad 1.7194999456$

$\mathrm{H} 1.20500004296 .9366998672 \quad 2.8101999760$

H $1.4742000103 \quad 4.62680006031 .9392999411$

H $3.0083999634 \quad 5.2585000992 \quad 2.4925000668$

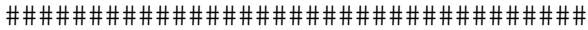

butylidene_ethylene

73

C $0.10100000352 \quad 0.12630000710-0.15289999545$

$\begin{array}{llll}\text { C } 2.6586999893 & 0.26350000501 & 0.29319998622\end{array}$

$\mathrm{Ru} 1.7994999886 \quad 1.8720999956 \quad 6.9999997504 \mathrm{E}-04$

Cl $1.8127000332 \quad 1.8317999840 \quad-2.4549999237$

$\begin{array}{lllll}\text { Cl } 1.4328000546 & 2.1010999680 & 2.4151999950\end{array}$

C $-0.451200008391 .3816000223-0.085900001228$

C $3.4677999020-0.57690000534-0.64179998636$

C $3.0639998913-2.0680000782-0.62739998102$

C $3.9892001152-2.9173998833-1.5052000284$

$\mathrm{H}-0.82709997892 \quad 1.7690000534 \quad 0.85500001907$

$\mathrm{H}-0.703899979591 .9034999609-1.0054999590$

H $0.16210000217-0.49259999394 \quad 0.73640000820$

$\mathrm{H} \quad 0.30489999056-0.33300000429-1.1138000488$

H $2.5573000908-0.15819999576 \quad 1.3049999475$

H $3.4449000359-0.17319999635-1.6565999985$

H $4.5075001717-0.50770002604-0.27649998665$

H $3.0782001019-2.4423999786 \quad 0.40459999442$

H $2.0311999321-2.1644999981-0.98299998045$

H $3.6879999638-3.9698998928-1.4934999943$

H $3.9686999321-2.5736000538-2.5450999737$

H $5.0267000198-2.8610000610-1.1561000347$

P $3.5996999741 \quad 3.4730999470 \quad 0.083400003612$

C $4.6318001747 \quad 3.41700005531 .6452000141$

$\begin{array}{llll}\text { C } 5.1958999634 & 2.0208001137 & 1.9822000265\end{array}$

C $5.8667998314 \quad 2.0250000954 \quad 3.3671998978$

C $6.9576001167 \quad 3.1015999317 \quad 3.4707999229$

C $6.4004001617 \quad 4.4892001152 \quad 3.1210000515$

C $5.7342000008 \quad 4.4948000908 \quad 1.7330000401$

C $4.67299985893 .5192000866-1.4639999866$

C $5.3515000343 \quad 4.8614997864-1.8203999996$

C $6.03179979324 .7746000290-3.2005000114$

C $7.0349998474 \quad 3.6147999763-3.2769999504$

C $6.37039995192 .2852001190-2.8921999931$

C $5.7045001984 \quad 2.3717000484-1.5080000162$

C $2.7829000950 \quad 5.16930007930 .11930000037$

C $1.7719000578 \quad 5.3042001724-1.0433000326$

C $1.1715999842 \quad 6.7186999321-1.0944000483$

C $0.51700001955 \quad 7.09840011600 .24169999361$

C $1.5068000555 \quad 6.94350004201 .4055000544$

C $2.1133999825 \quad 5.5292000771 \quad 1.4634000063$

H $3.8722000122 \quad 3.62409996992 .4100999832$

H $4.3826999664 \quad 1.2906999588 \quad 1.9726999998$

H $5.93060016631 .7130000591 \quad 1.2276999950$

H $5.0987000465 \quad 2.2065999508 \quad 4.1312999725$

H $6.2883000374 \quad 1.0336999893 \quad 3.5745000839$

H $7.3920001984 \quad 3.1094000340 \quad 4.4777998924$ 
H $7.7762999535 \quad 2.8571000099 \quad 2.7778999805$

H $5.6586999893 \quad 4.7849001884 \quad 3.8761999607$

$\mathrm{H} 7.19750022895 .2424998283 \quad 3.1503000259$

H $6.5008997917 \quad 4.2997999191 \quad 0.97219997644$

H $5.3270998001 \quad 5.4903998375 \quad 1.5219999552$

$\mathrm{H} 3.92650008203 .3006000519-2.2386000156$

H $4.62260007865 .6772999763-1.8402999640$

$\mathrm{H} \quad 6.10160017015 .1241002083-1.0637999773$

H $5.25820016864 .6350002289-3.9684998989$

H $6.52860021595 .7269001007-3.4240999222$

$\mathrm{H} 7.46470022203 .5508000851-4.2841000557$

H $7.8715000153 \quad 3.8131999969-2.5906999111$

H $5.60659980772 .0257000923-3.6377000809$

$\mathrm{H} \quad 7.1080999374 \quad 1.4730999470 \quad-2.8977999687$

H $6.4815001488 \quad 2.5394999981-0.75040000677$

H $5.22289991381 .4197000265-1.2711000443$

H $3.59290003785 .8916001320-0.049199998379$

$\mathrm{H} 2.23309993745 .0503001213-2.0027000904$

$\mathrm{H} \quad 0.96090000868 \quad 4.5753002167-0.90399998426$

H $1.96920001517 .4394001961-1.3281999826$

$\mathrm{H} \quad 0.444200009116 .7806000710-1.9127000570$

$\mathrm{H} \quad 0.13369999826 \quad 8.1252002716 \quad 0.20110000670$

$\mathrm{H}-0.349900007256 .4450998306 \quad 0.41600000858$

$\mathrm{H} 2.31640005117 .6796998978 \quad 1.2913000584$

$\mathrm{H} 1.01400005827 .1666002274 \quad 2.3594000340$

$\mathrm{H} 1.3403999805 \quad 4.7916002274 \quad 1.7060999870$

H 2.8412001133 5.4865999222 2.2802000046

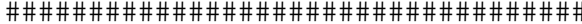

eoctene

24

C $0.018100000918 \quad 1.6256999969-0.026300000027$

C $5.0000002375 \mathrm{E}-04 \quad 0.0948999971150 .019799999893$

C $-1.4244999886-0.49140000343-0.010099999607$

C $-1.4398000240-1.99549996850 .0041999998502$

C $-1.9749000072-2.7539999485 \quad 0.96549999714$

C $-1.9558000565-4.25769996641 .0001000166$

C $-1.1851999760-4.8207998276 \quad 2.2102000713$

C $-1.1921000481-6.35139989852 .2639000416$

$\mathrm{H} \quad 1.0411000252 \quad 2.0153999329-0.0030000000261$

$\mathrm{H}-0.46090000868 \quad 2.0004999638-0.93830001354$

$\mathrm{H}-0.520200014112 .05329990390 .82760000229$

$\mathrm{H} 0.57340002060-0.30660000443-0.82700002193$

$\mathrm{H} \quad 0.50940001011-0.25740000606 \quad 0.92599999905$

$\mathrm{H}-1.9960000515-0.107799999420 .84530001879$

$\mathrm{H}-1.9336999655-0.12330000103-0.91420000792$

$\mathrm{H}-0.95230001211-2.4869999886-0.84109997749$

$\mathrm{H}-2.4549999237-2.2622001171 \quad 1.8149000406$

$\mathrm{H}-2.9870998859-4.6416997910 \quad 1.0345000029$

$\mathrm{H}-1.5124000311-4.64330005650 .072499997914$

$\mathrm{H}-0.15189999342-4.45270013812 .1742999554$

$\mathrm{H}-1.6203999519-4.41680002213 .1342999935$

$\mathrm{H}-0.63700002432-6.7242999077 \quad 3.1310999393$

$\mathrm{H}-2.2144999504-6.7411999702 \quad 2.3296000957$

$\mathrm{H}-0.73339998722-6.7807002068 \quad 1.3657000065$

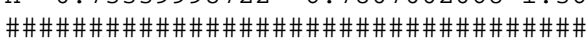

ethylene

6

C $-0.0089999996126 \quad 0.66530001163 \quad 0.0032999999821$

C $0.0089999996126-0.66530001163-0.0032999999821$

$\mathrm{H} 0.90710002184 \quad 1.2504999638-0.0019000000320$

$\mathrm{H}-0.94069999456 \quad 1.2252999544 \quad 0.013399999589$

$\mathrm{H}-0.90710002184-1.2504999638 \quad 0.0019000000320$

H $0.94069999456-1.2252999544-0.013399999589$

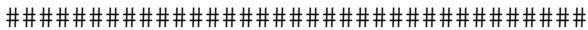

methylidene-P

110

Ru $0.90539997816-0.35040000081-0.78060001135$

Cl $0.77450001240 \quad-0.27169999480 \quad 1.6820000410$ 
Cl $0.78250002861-0.52619999647-3.2353999615$

$\begin{array}{lllll}\text { C } 2.5762999058 & 0.35359999537 & -0.76050001383\end{array}$

P $1.5176000595-2.7239999771-0.65939998627$

H $3.1610000134 \quad 0.50050002337-1.6759999990$

C $-0.031500000507-3.7783000469-0.49459999800$

C $2.3097000122-3.3627998829-2.2367000580$

C $2.5230000019-3.16779994960 .86400002241$

C $3.5608999729-2.5708000660-2.6721999645$

$\begin{array}{llll}\text { C } 3.9855999947 & -2.9767999649 & -4.0940999985\end{array}$

C $4.2265000343-4.4896001816-4.2101001740$

C $3.0009999275-5.2855000496-3.7360999584$

C $2.5801999569-4.8807001114-2.3106999397$

C $-0.98900002241-3.6092000008-1.6950999498$

C $-2.2030999660-4.5464000702-1.5686999559$

C $-2.9470000267-4.3446002007-0.23989999294$

C $-1.9939999580-4.4769001007 \quad 0.95730000734$

C $-0.78619998693-3.53150010110 .83120000362$

C $2.6050999165-4.66429996491 .2404999733$

C $3.2532999516-4.8326997757 \quad 2.6280000210$

C $4.6417999268-4.17799997332 .6907999516$

$\begin{array}{lllll}\text { C } 4.5830998421 & -2.7037999630 & 2.2611999512\end{array}$

C $3.9354000092-2.54789996150 .87430000305$

H $0.31690001488-4.8204998970-0.49149999022$

H $1.5336999893-3.1110999584-2.9714999199$

H $1.9503999949-2.6458001137 \quad 1.6427999735$

H $3.3422000408-1.5025000572-2.6505999565$

H $4.3906998634-2.7623000145-1.9794000387$

H $3.1944999695-2.6733000278-4.7932000160$

H $4.8888001442-2.4240000248-4.3814001083$

H $4.4773998260-4.7593998909-5.2435002327$

H $5.0945000648-4.7667999268-3.5936000347$

H $2.1610000134-5.1027998924-4.4212999344$

H $3.2063999176-6.3629999161-3.7693998814$

$\mathrm{H} 1.6992000341-5.4594001770-2.0116999149$

H $3.3856000900-5.1477999687-1.6147999763$

$\mathrm{H}-1.3287999630-2.5666000843-1.7474999428$

$\mathrm{H}-0.47319999337-3.8029000759-2.6405000687$

H $-1.8588000536-5.5887999535-1.6368999481$

$\mathrm{H}-2.8810999393-4.3857002258-2.4159998894$

$\mathrm{H}-3.3984999657-3.3422999382-0.22830000520$

$\mathrm{H}-3.7734999657-5.0606999397-0.15320000052$

H $-2.5258998871-4.27050018311 .8940999508$

$\mathrm{H}-1.6351000071-5.5148000717 \quad 1.0227999687$

$\mathrm{H}-0.12349999696-3.6635999680 \quad 1.6915999651$

$\mathrm{H}-1.1209000349-2.4885001183 \quad 0.87569999695$

H $3.2039000988-5.2062997818 \quad 0.49840000272$

H $1.6145999432 \quad-5.1297998428 \quad 1.2462999821$

H $3.3217000961-5.8993000984 \quad 2.8763999939$

H $2.6003000736-4.37370014193 .3835999966$

H $5.3259000778-4.72130012512 .0222001076$

H $5.0570998192-4.2655000687 \quad 3.7023000717$

н $3.9964001179-2.1293001175 \quad 2.9909999371$

H $5.5906000137-2.2695000172 \quad 2.2514998913$

H $3.8877000809-1.48919999600 .60310000181$

H $4.5669999123-3.04679989810 .12749999762$

P $-0.37310001254 \quad 1.7410000563-0.93580001593$

C $-0.074900001287 \quad 2.7135999203-2.5118000507$

C $-2.2181999683 \quad 1.3680000305-0.99159997702$

C $-0.17739999294 \quad 2.8187000751 \quad 0.59130001068$

C $-2.6865000725 \quad 0.72630000114-2.3162999153$

C $-4.2088999748 \quad 0.49419999123 \quad-2.3039000034$

C $-4.6529998779-0.35130000114-1.1013000011$

$\begin{array}{llll}\text { C }-4.1663999557 & 0.26300001144 & 0.21960000694\end{array}$

C $-2.64590001110 .49349999428 \quad 0.20870000124$

C $1.4133000374 \quad 2.9539000988-2.8415000439$

C $1.5622999668 \quad 3.5608000755-4.2476000786$

C $0.74589997530 \quad 4.8526000977-4.4057002068$

C $-0.73089998960 \quad 4.6212000847-4.0520000458$

C $-0.88559997082 \quad 4.0202999115-2.6428000927$

C $1.2056000233 \quad 3.4983999729 \quad 0.66710001230$

C $1.4289000034 \quad 4.1194000244 \quad 2.0566000938$ 
C $0.31720000505 \quad 5.1202998161 \quad 2.4068999290$ C $-1.0707999468 \quad 4.4732999802 \quad 2.2866001129$

C $-1.2934000492 \quad 3.84120011330 .89880001545$

$\mathrm{H}-0.42779999971 \quad 2.0021998882 \quad-3.2693998814$

$\mathrm{H}-2.7235999107 \quad 2.3392000198 \quad-0.90039998293$

$\mathrm{H}-0.19020000100 \quad 2.0566000938 \quad 1.3815000057$

$\mathrm{H}-2.42860007291 .3619999886-3.1688001156$

H $-2.1598999500-0.22229999304-2.4765999317$

$\mathrm{H}-4.72009992601 .4677000046-2.2697000504$

$\mathrm{H}-4.51350021360 .014200000092-3.2420001030$

$\mathrm{H}-5.7445001602-0.46129998565-1.0929000378$

$\mathrm{H}-4.2367000580-1.3638000488-1.2023999691$

$\mathrm{H}-4.67619991301 .22360002990 .38479998708$

$\mathrm{H}-4.4345998764-0.38260000944 \quad 1.0650000572$

H $-2.3234000206 \quad 0.936900019651 .1561000347$

H $-2.1312999725 \quad-0.474400013690 .14730000496$

$\mathrm{H} 1.8640999794 \quad 3.6312000751-2.1050999165$

H $1.94770002372 .0041000843-2.7983000278$

H $2.6224000454 \quad 3.7514998913-4.4559998512$

H $1.22469997412 .8203001022-4.9858999252$

H $1.1576999426 \quad 5.6256999969-3.7400999069$

H $0.83509999514 \quad 5.2392997742 \quad-5.4284000397$

$\mathrm{H}-1.2942999601 \quad 5.5605998039-4.1178998947$

$\mathrm{H}-1.1768000126 \quad 3.9335000515-4.7846999168$

H $-1.9472999573 \quad 3.8503999710 \quad-2.4295001030$

$\mathrm{H}-0.52819997072 \quad 4.7500000000-1.9048000574$

H $1.99650001532 .7725999355 \quad 0.45350000262$

H $1.2755999565 \quad 4.2883000374 \quad-0.092699997127$

H $2.4094998837 \quad 4.61049985892 .0908999443$

H $1.4500000477 \quad 3.31399989132 .8032000065$

$\mathrm{H} \quad 0.37560001016 \quad 5.9791002274 \quad 1.7218999863$

H 0.464199990035 .51620006563 .4193000793

$\mathrm{H}-1.1728999615 \quad 3.6909999847 \quad 3.0518999100$

$\mathrm{H}-1.8568999767 \quad 5.2128000259 \quad 2.4848999977$

$\mathrm{H}-2.2794001102 \quad 3.3659000397 \quad 0.88010001183$

H $-1.3084000349 \quad 4.6325998306 \quad 0.13899999857$

H $3.0520000458 \quad 0.652700006960 .18080000579$

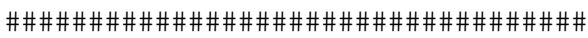

methylidene-eoctene-TS

82

C $5.0000002375 \mathrm{E}-04 \quad 0.0542000010610 .030300000682$

C $2.20009994510 .0194000005723 .9999998990 \mathrm{E}-04$

$\mathrm{Ru} 0.59320002794 \quad 1.8016999960-0.0041999998502$

Cl $0.645600020891 .6921000481-2.4567999840$

$\mathrm{Cl} 0.602800011631 .9076000452 \quad 2.4511001110$

C $2.7005999088 \quad 1.3423000574 \quad 0.19460000098$

C $2.3731999397-1.0089999437 \quad 1.1125999689$

C $1.8725999594-2.4210999012 \quad 0.78250002861$

C $2.1693000793-3.41820001601 .9069000483$

C $3.52900004392 .0782001019-0.84030002356$

C $5.03690004351 .8344999552-0.62000000477$

C $5.8990998268 \quad 2.6219000816-1.6116000414$

$\mathrm{H}-0.24420000613-0.433499991890 .97670000792$

$\mathrm{H}-0.22759999335-0.48600000143-0.89259999990$

H $2.2298998833-0.35030001402-1.0247000456$

H $2.9495999813 \quad 1.5845999718 \quad 1.2287000418$

H $3.4542000294-1.0674999952 \quad 1.3092000484$

H $1.9193999767 \quad-0.62589997053 \quad 2.0339000225$

H $0.79339998960 \quad-2.4037001133 \quad 0.58829998970$

H $2.3445999622-2.7625999451-0.14910000563$

H $1.8166999817-4.42250013351 .6510000229$

H $3.2451000214-3.48210000992 .1066999435$

H $1.6775000095-3.1171000004 \quad 2.8387999535$

H $3.23329997061 .7812000513-1.8487999439$

H $3.3412001133 \quad 3.1584000587 \quad-0.76289999485$

H 5.30840015412 .11010003090 .40779998899

H $5.2451000214 \quad 0.76059997082 \quad-0.71749997139$

H $6.9657001495 \quad 2.4363000393-1.4470000267$

H $5.6644001007 \quad 2.3443000317-2.6451001167$

H $5.7277998924 \quad 3.6998999119-1.5117000341$ 


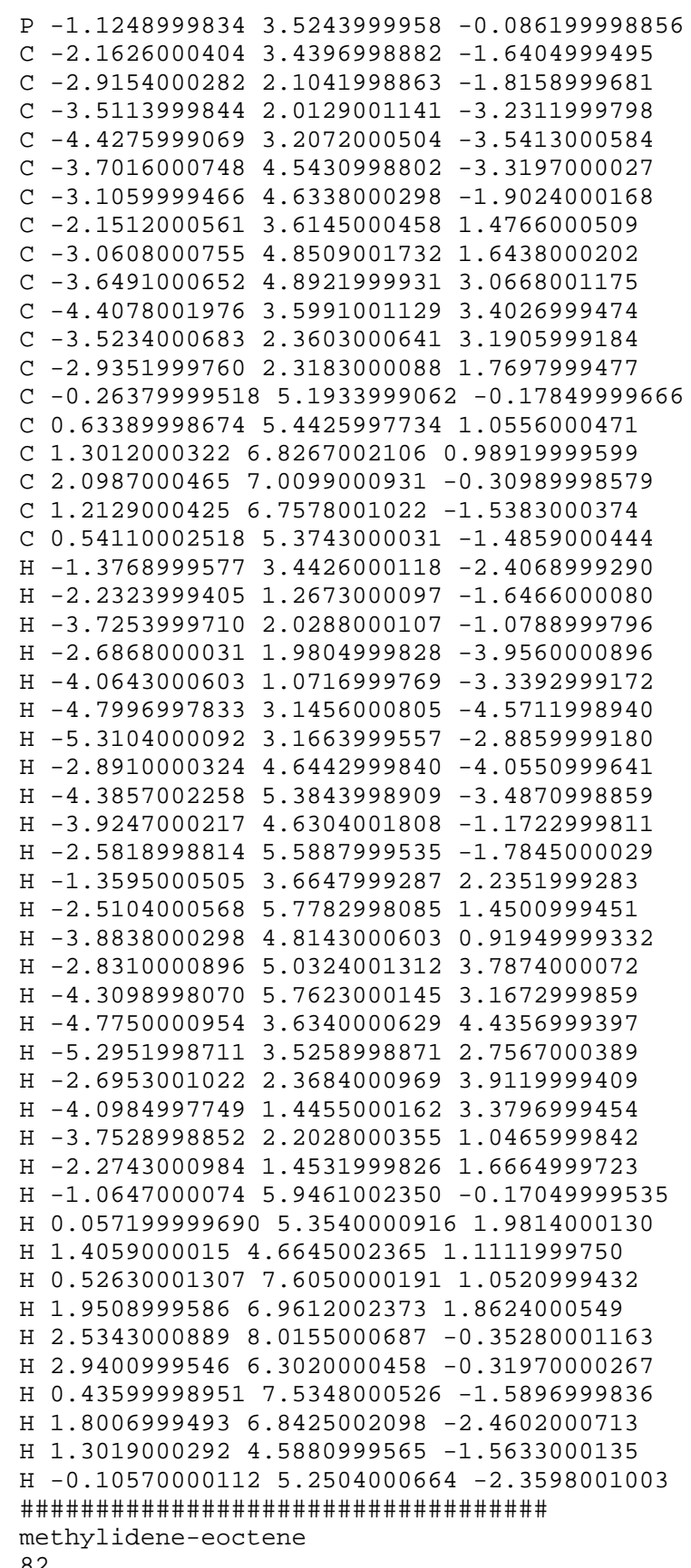
82

C $-0.24379999936 \quad 0.32370001078 \quad 0.27599999309$ C $2.80180001260 .18600000441-0.042399998754$ Ru $0.79879999161 \quad 1.79779994490 .074799999595$ Cl $0.84420001507 \quad 1.5427000523-2.3708999157$ Cl $0.888800024992 .0882999897 \quad 2.5169000626$ C $3.1354000568 \quad 1.4452999830 \quad 0.37979999185$ C $2.7388000488-0.99980002642 \quad 0.88999998569$

C $1.9345999956-2.1975998878 \quad 0.37090000510$ C $1.9681999683-3.39229989051 .3282999992$ C $3.63280010222 .5492000580-0.52499997616$ $\begin{array}{lllll}\text { C } 5.1444997787 & 2.7978999615 & -0.32859998941\end{array}$ C $5.66160011293 .9375998974-1.2117999792$ $\mathrm{H}-0.46909999847-0.0881000012161 .2657999992$ $\mathrm{H}-0.64249998331-0.20960000157-0.59399998188$ H 2.7495999336-0.0080000003800 -1.1130000353 
H 3.27239990231 .60580003261 .4477000237

H $3.7787001133-1.33029997351 .0470999479$

H $2.3715999126-0.67479997873 \quad 1.8707000017$

$\mathrm{H} \quad 0.89349997044-1.89279997350 .20620000362$

H $2.3211998940-2.4958000183-0.61280000210$

H $1.3882999420-4.2330999374 \quad 0.93459999561$

H $2.9939000607-3.7418999672 \quad 1.4929000139$

H $1.5492999554 \quad-3.1254000664 \quad 2.3052000999$

H $3.41589999202 .3106000423-1.5703999996$

H $3.1096000671 \quad 3.4914999008-0.29499998689$

H $5.3431000710 \quad 3.0227000713 \quad 0.72729998827$

H $5.6861000061 \quad 1.8710999489-0.55720001459$

H $6.7360000610 \quad 4.0938000679-1.0702999830$

H $5.4899001122 \quad 3.7232000828-2.2723999023$

H $5.1532001495 \quad 4.8801999092-0.97759997845$

P $-0.97479999065 \quad 3.4177000523-0.10459999740$

C $-2.0371999741 \quad 3.2500000000-1.6426000595$

C $-2.8410000801 \quad 1.9357000589-1.7157000303$

C $-3.46849989891 .7644000053-3.1105000973$

C $-4.3529000282 \quad 2.9628999233-3.4862000942$

C $-3.5764999390 \quad 4.2829999924 \quad-3.3719999790$

C $-2.94880008704 .4523000717-1.9756000042$

C $-1.99520003803 .5957999229 \quad 1.4601999521$

C $-2.9038000107 \quad 4.84200000761 .5558999777$

C $-3.4781000614 \quad 4.97930002212 .9786999226$

C $-4.2379999161 \quad 3.7153000832 \quad 3.4075000286$

C $-3.3585999012 \quad 2.4633998871 \quad 3.2665998936$

$\begin{array}{llll}\text { C }-2.7860999107 & 2.3289999962 & 1.8446999788\end{array}$

C $-0.17489999533 \quad 5.1107997894-0.30480000377$

C $0.70289999247 \quad 5.49910020830 .90630000830$

C $1.2827999592 \quad 6.9145998955 \quad 0.73869997263$

C $2.06360006337 .0584998131-0.57569998503$

C $1.1950000525 \quad 6.6648001671-1.7790000439$

C $0.61489999294 \quad 5.2474999428-1.6261999607$

$\mathrm{H}-1.26289999493 .1737000942 \quad-2.4168999195$

H $-2.18899989131 .0861999989-1.5008000135$

$\mathrm{H}-3.6391000748 \quad 1.9398000240-0.96249997616$

$\mathrm{H}-2.66199994091 .6552000046-3.8480999470$

$\mathrm{H}-4.0520000458 \quad 0.83609998226-3.1407001019$

$\mathrm{H}-4.7491998672 \quad 2.8424000740 \quad-4.5018000603$

$\mathrm{H}-5.2217998505 \quad 2.9976000786-2.8125000000$

$\mathrm{H}-2.7785000801 \quad 4.3034000397-4.1273999214$

$\mathrm{H}-4.2340998650 \quad 5.1350998878-3.5850999355$

$\mathrm{H}-3.7525999546 \quad 4.5286002159-1.2332999706$

$\mathrm{H}-2.3952999115 \quad 5.3962998390 \quad-1.9391000271$

$\mathrm{H}-1.1983000040 \quad 3.6919999123 \quad 2.2088000774$

$\mathrm{H}-2.3577001095 \quad 5.7553000450 \quad 1.2970000505$

$\mathrm{H}-3.7346000671 \quad 4.7572999001 \quad 0.84469997883$

$\mathrm{H}-2.6524000168 \quad 5.1637001038 \quad 3.6802999973$

$\mathrm{H}-4.13460016255 .8571000099 \quad 3.0271000862$

$\mathrm{H}-4.5934000015 \quad 3.8187000751 \quad 4.4398999214$

H $-5.1326999664 \quad 3.6029000282 \quad 2.7774999142$

$\mathrm{H}-2.52320003512 .5162000656 \quad 3.9774999619$

$\mathrm{H}-3.9330999851 \quad 1.56400001053 .5195999146$

$\mathrm{H}-3.61249995232 .17319989201 .1394000053$

$\mathrm{H}-2.13669991491 .4517999887 \quad 1.7914999723$

$\mathrm{H}-1.01250004775 .8206000328-0.34490001202$

H $0.12970000505 \quad 5.4430999756 \quad 1.8367999792$

H $1.5195000172 \quad 4.7754001617 \quad 1.0195000172$

H $0.46079999208 \quad 7.64550018310 .75309997797$

$\mathrm{H} 1.9253000021 \quad 7.15010023121 .5957000256$

H $2.43289995198 .0847997665-0.69059997797$

H $2.9498000145 \quad 6.4082999229-0.54210001230$

H $0.36930000782 \quad 7.3846001625-1.8796999454$

H $1.77540004256 .7234001160-2.7076001167$

$\mathrm{H} 1.4244999886 \quad 4.5090999603-1.6535999775$

H $-0.023399999365 \quad 5.0198998451-2.4853000641$

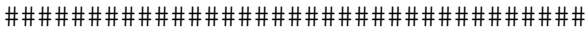

methylidene-ethylene-TS

64 
C $-0.11339999735 \quad 0.17620000243 \quad 0.10890000314$

C $2.0731000900 \quad 0.052700001746-0.099899999797$

$\mathrm{Ru} 1.5881999731 \quad 1.8286000490 \quad 0.063000001013$

$\begin{array}{lllll}\text { Cl } 1.2516000271 & 2.0283000469 & -2.3561999798\end{array}$

Cl 1.70079994201 .69050002102 .5065000057

C $-0.55540001392 \quad 1.5089999437 \quad 0.24199999869$

$\mathrm{H}-0.81669998169 \quad 1.8717000484 \quad 1.2318999767$

$\mathrm{H}-0.988200008872 .0000000000-0.62500000000$

$\mathrm{H}-0.058400001377-0.442999988790 .99790000916$

$\mathrm{H}-0.22930000722-0.31430000067-0.85170000792$

H $2.1661000252-0.42179998755-1.0808999538$

H $2.3441998959-0.540700018410 .77780002356$

P $3.47930002213 .3471000195-0.030400000513$

C $4.5848999023 \quad 3.21320009231 .4779000282$

C 5.31360006331 .85580003261 .5673999786

C $5.9829998016 \quad 1.69270002842 .9428000450$

C 6.94570016862 .85170006753 .2434000969

C 6.23810005194 .20930004123 .1173000336

C $5.5651998520 \quad 4.3773999214 \quad 1.7415000200$

C $4.4232997894 \quad 3.2464001179-1.6426000595$

C $5.5571999550 \quad 4.2793002129-1.8177000284$

C $6.1001000404 \quad 4.2462000847 \quad-3.2581999302$

C $6.57460021972 .8378999233-3.6475000381$

C $5.46470022201 .7965999842-3.4344000816$

C $4.91800022131 .8279000521-1.9961999655$

C 2.80920004845 .10209989550 .030999999493

C $2.0546000004 \quad 5.5159997940-1.2520999908$

C $1.55009996896 .9667000771-1.1471999884$

C $0.67580002546 \quad 7.1817998886 \quad 0.096799999475$

C $1.41960000996 .7589998245 \quad 1.3719999790$

C $1.91980004315 .3080000877 \quad 1.2793999910$

H $3.8340001106 \quad 3.20869994162 .2787001133$

H 4.60370016101 .03980004791 .4005999565

H $6.0822000504 \quad 1.79260003570 .78579998016$

H $5.20020008091 .6528999805 \quad 3.7118999958$

H $6.5156998634 \quad 0.73479998112 \quad 2.9834001064$

H $7.37610006332 .7409999371 \quad 4.2459998131$

H $7.7870001793 \quad 2.81699991232 .5355999470$

H $5.4727001190 \quad 4.2943000793 \quad 3.9012000561$

H $6.9488000870 \quad 5.02890014653 .2806999683$

H $6.3400998116 \quad 4.4050998688 \quad 0.96630001068$

H $5.0501999855 \quad 5.34340000151 .7080999613$

H $3.6254999638 \quad 3.4730000496-2.3619000912$

H $5.21409988405 .2912001610-1.5737999678$

H $6.3766999245 \quad 4.0475997925-1.1261999607$

H $5.3070001602 \quad 4.5679998398-3.9475998878$

H $6.9189000130 \quad 4.9692001343-3.3603999615$

H $6.9134998322 \quad 2.8273000717-4.6904001236$

H $7.44589996342 .5701000690-3.0320000648$

H $4.6368999481 \quad 1.9933999777-4.1289000511$

H $5.8372001648 \quad 0.79129999876 \quad-3.6652998924$

H $5.71000003811 .5190999508-1.3022999763$

H $4.0939002037 \quad 1.1168999672 \quad-1.8983000517$

H $3.6881999969 \quad 5.7543001175 \quad 0.12759999931$

H $2.7033998966 \quad 5.4223999977-2.1287000179$

H $1.2127000093 \quad 4.8345999718-1.4247000217$

H 2.4135000706 7.6468000412 -1.1043000221

$\mathrm{H} \quad 0.99409997463 \quad 7.2245998383-2.0564000607$

H $0.36379998922 \quad 8.2307996750 \quad 0.16680000722$

$\mathrm{H}-0.24300000072 \quad 6.5855998993 \quad 3.9999998990 \mathrm{E}-04$

H $2.27900004397 .4267997742 \quad 1.5307999849$

H $0.77240002155 \quad 6.8678002357 \quad 2.2502999306$

$\mathrm{H} 1.0580999851 \quad 4.62760019301 .2286000252$

H $2.4572000504 \quad 5.03660011292 .1933999062$

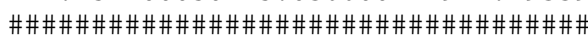

methylidene-pentene-12-TS

73

C $-0.049100000411 \quad 6.9999997504 \mathrm{E}-04 \quad 0.041400000453$

C $1.9455000162-0.053500000387-0.094599999487$

$\mathrm{Ru} 1.5180000067 \quad 1.77269995210 .0063999998383$ 
Cl $1.2538000345 \quad 1.7948999405 \quad-2.4238998890$ Cl $1.6883000135 \quad 1.7167999744 \quad 2.4463000298$ C $-0.515600025651 .3826999664 \quad 0.14929999411$ C $-0.33649998903-0.93910002708 \quad 1.2086000443$ $\begin{array}{lllll}\text { C } & 0.019099999219 & -2.4091999531 & 0.95329999924\end{array}$ C $-0.36640000343-3.3094999790 \quad 2.1308999062$

$\mathrm{H}-0.89310002327 \quad 1.6936999559 \quad 1.1217999458$

$\mathrm{H}-0.99919998646 \quad 1.8071000576-0.72689998150$

$\mathrm{H}-0.18619999290 \quad-0.41170001030-0.95819997787$

H $2.1312000751-0.53250002861-1.0573999882$

H $2.2822999954-0.572300016880 .80409997702$

H $0.16500000656-0.558399975302 .1052999496$

H $-1.4158999920-0.87389999628 \quad 1.4078999758$

$\mathrm{H}-0.48980000615-2.7532999516 \quad 0.042300000787$

H $1.0940999985-2.50690007210 .75800001621$

$\mathrm{H}-0.11599999666-4.3561000824 \quad 1.9299000502$

H $0.15899999440-3.0078999996 \quad 3.0439000130$

H $-1.4419000149-3.2562999725 \quad 2.3355000019$

P $3.1730999947 \quad 3.5703999996-0.078299999237$

C $3.1224000454 \quad 4.5349998474-1.6822999716$

C $1.80040001875 .3095002174-1.8722000122$

C $1.70000004775 .8530001640-3.3073999882$

C $2.90680003176 .7350001335-3.6619999409$

C $4.23029994965 .9902000427-3.4307999611$

C $4.3334999084 \quad 5.4400000572-1.9954999685$

C $3.0341999531 \quad 4.6694002151 \quad 1.4273999929$

C $4.0771999359 \quad 5.7994999886 \quad 1.5493999720$

C $3.9797000885 \quad 6.4702000618 \quad 2.9321999550$

C $2.5610001087 \quad 6.9925999641 \quad 3.2065000534$

C $1.51250004775 .8811001778 \quad 3.0425000191$

C 1.60609996325 .21049976351 .6612000465

$\begin{array}{lllll}\text { C } 4.9019999504 & 2.8478000164 & -0.011199999601\end{array}$

C 5.21059989932 .12759995461 .3186999559

C $6.6563000679 \quad 1.6000000238 \quad 1.3329999447$

C $6.9416999817 \quad 0.68480002880 \quad 0.13369999826$

C $6.6241998672 \quad 1.3955999613-1.1895999908$

C $5.17959976201 .9230999947-1.2172000408$

H $3.0996000767 \quad 3.7172999382-2.4145998955$

H $0.94859999418 \quad 4.6543998718-1.6655999422$

$\mathrm{H} 1.75680005556 .1505999565-1.1682000160$

$\mathrm{H} 1.64310002335 .0032000542-4.0005002022$

H $0.76740002632 \quad 6.4184999466-3.4231998920$

H $2.8405001163 \quad 7.0720000267-4.7035999298$

H $2.88940000537 .6406002045-3.0376999378$

$\mathrm{H} 4.30520009995 .1531000137-4.1388998032$

H $5.0826997757 \quad 6.6508002281-3.6326999664$

H $4.3699002266 \quad 6.2800998688-1.2913000584$

H $5.2764000893 \quad 4.8938999176-1.8848999739$

H $3.2083001137 \quad 3.9398999214 \quad 2.2286000252$

H 5.09369993215 .41919994351 .3938000202

H $3.8986001015 \quad 6.5553002357 \quad 0.77410000563$

H $4.2546000481 \quad 5.7381000519 \quad 3.7044999599$

H $4.7088999748 \quad 7.2871999741 \quad 2.9981999397$

H $2.5053999424 \quad 7.4233999252 \quad 4.2136001587$

H 2.3366999626 7.80849981312 .5037999153

H $1.6611000299 \quad 5.1149997711 \quad 3.8152999878$

$\mathrm{H} 0.50340002775 \quad 6.2852997780 \quad 3.1884999275$

$\mathrm{H} 1.3450000286 \quad 5.94140005110 .88650000095$

H $0.88389998674 \quad 4.3902997971 \quad 1.6030999422$

H $5.57170009613 .7167000771-0.088100001216$

H 5.05989980702 .80080008512 .1684000492

$\mathrm{H} \quad 4.50649976731 .2999999523 \quad 1.4593000412$

H 7.3534002304 2.4507000446 1.3133000135

H $6.8392000198 \quad 1.0680999756 \quad 2.2743000984$

H $7.9869999886 \quad 0.35280001163 \quad 0.14710000157$

H $6.3224000931-0.219999998810 .21469999850$

H $7.31990003592 .2365000248-1.3265999556$

H $6.7811999321 \quad 0.71899998188-2.0381999016$

H $4.47879981991 .0796999931-1.1907000542$

H $4.99399995802 .4451000690-2.1614000797$

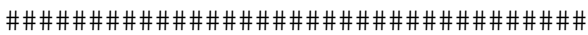




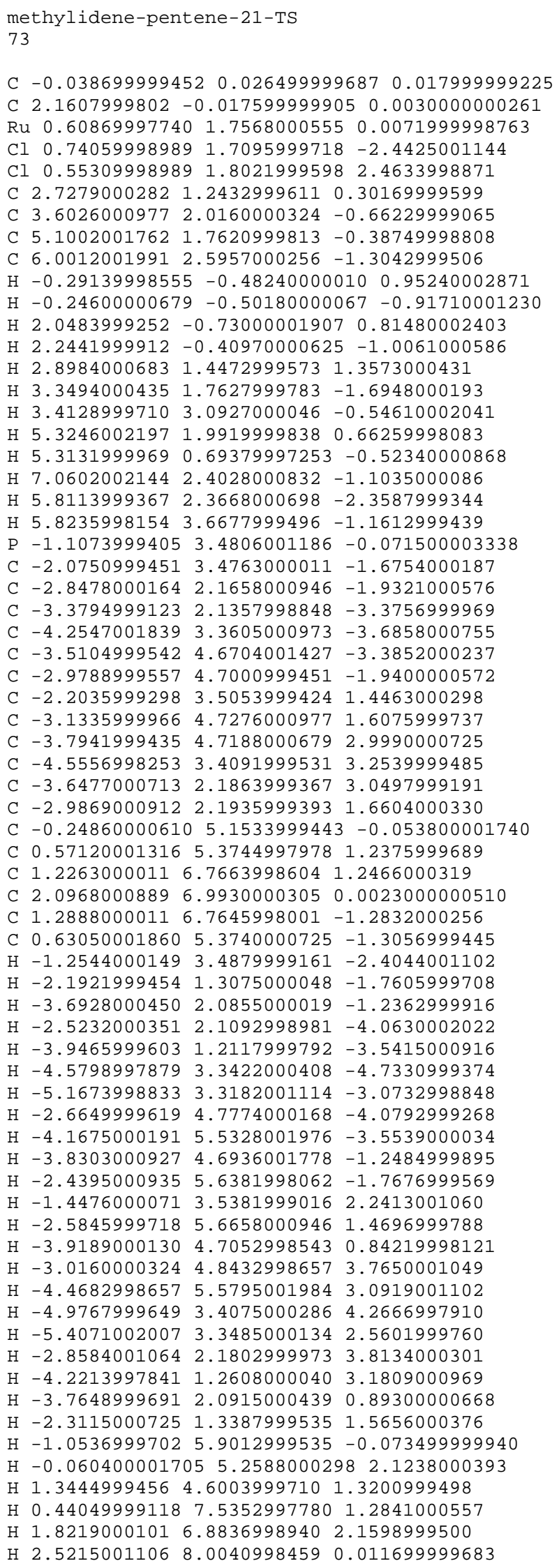


H $2.9463000298 \quad 6.29510021210 .024100000039$

$\mathrm{H} \quad 0.50720000267 \quad 7.5345997810-1.3609999418$

$\mathrm{H} 1.9291000366 \quad 6.8794999123-2.1659998894$

H $1.4042999744 \quad 4.5974001884 \quad-1.3550000191$

H $0.039599999785 \quad 5.2635998726 \quad-2.2200999260$

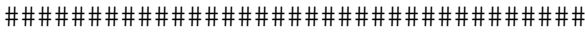

methylidene-zoctene-TS

82

C $-0.0051000001840 \quad 0.094599999487 \quad-0.029899999499$

C $2.19409990310 .051199998707 \quad 0.013000000268$

$\mathrm{Ru} 0.581200003621 .84319996830 .0031000000890$

Cl $0.63370001316 \quad 1.9351999760-2.4498000145$

Cl $0.60740000010 \quad 1.7777999640 \quad 2.4607000351$

C 2.70639991761 .37160003190 .18790000677

C $2.4886999130-0.77689999342-1.2259000540$

C $2.0778000355-2.2504000664-1.1059999466$

C $2.4240999222-3.0524001122-2.3640000820$

C $3.5789999962 \quad 2.0813999176-0.82630002499$

C $5.0777997971 \quad 1.8775999546-0.51859998703$

C $5.9758000374 \quad 2.6328999996-1.5037000179$

$\mathrm{H}-0.25429999828 \quad-0.44969999790 \quad 0.88529998064$

$\mathrm{H}-0.22280000150-0.39640000463-0.98189997673$

H $2.0638000965-0.496699988840 .94520002604$

H $2.8831999302 \quad 1.6548999548 \quad 1.2243000269$

H $2.0229001045-0.31130000949-2.1012001038$

H $3.5736999512-0.73150002956-1.3997999430$

H $2.5725998878-2.6974000931-0.23280000687$

H $0.99930000305-2.3210999966-0.91509997845$

H $2.1335999966-4.1027002335-2.2599999905$

H $1.9084999561-2.6463999748-3.2416000366$

H $3.5002999306-3.0216000080-2.5694000721$

H $3.34270000461 .7589999437-1.8423000574$

H $3.36730003363 .1587998867-0.79409998655$

H 5.28709983832 .21110010150 .50639998913

H $5.31479978560 .80580002069-0.54640001059$

H $7.03569984442 .4834001064-1.2726999521$

H $5.8045997620 \quad 2.2959001064 \quad-2.5320999622$

H $5.7747998238 \quad 3.7098999023-1.4720000029$

P $-1.1684000492 \quad 3.5318999290 \quad 0.031300000846$

C $-2.22429990773 .5248000622-1.5139000416$

C $-2.94959998132 .1865999699-1.7663999796$

C $-3.56579995162 .1740999222-3.1761999130$

C $-4.5121998787 \quad 3.3650999069-3.3949000835$

C $-3.8108000755 \quad 4.6993999481 \quad-3.0978999138$

C $-3.1963000298 \quad 4.7119002342 \quad-1.6858999729$

C $-2.1767001152 \quad 3.5088000298 \quad 1.6081999540$

C $-3.1068000793 \quad 4.7147002220 \quad 1.8618999720$

C $-3.6795001030 \quad 4.65520000463 .2904999256$

C $-4.4106998444 \quad 3.3296000957 \quad 3.5517001152$

C $-3.5060999393 \quad 2.1238000393 \quad 3.2537000179$

C $-2.93249988562 .1819000244 \quad 1.8276000023$

C $-0.33770000935 \quad 5.2185997963 \quad 0.029500000179$

C $0.59960001707 \quad 5.3901000023 \quad 1.2472000122$

C $1.2439999580 \quad 6.7866001129 \quad 1.2625999451$

C $1.98919999607 .0822000504-0.046900000423$

C $1.0618000031 \quad 6.9075999260-1.2578999996$

C $0.41690000892 \quad 5.5107998848-1.2874000072$

H $-1.4487999678 \quad 3.5947999954 \quad-2.2871000767$

$\mathrm{H}-2.24449992181 .3572000265-1.6619000435$

$\mathrm{H}-3.74559998512 .0432000160 \quad-1.0243999958$

H $-2.7525999546 \quad 2.2079999447-3.9138000011$

$\mathrm{H}-4.0998997688 \quad 1.2296999693 \quad-3.3378000259$

$\mathrm{H}-4.8994998932 \quad 3.3615000248-4.4211001396$

$\mathrm{H}-5.3831000328 \quad 3.2623999119-2.7307000160$

$\mathrm{H}-3.0139000416 \quad 4.8652000427-3.8364999294$

$\mathrm{H}-4.51499986655 .5345001221-3.2014000416$

$\mathrm{H}-4.0043001175 \quad 4.6434001923-0.94739997387$

$\mathrm{H}-2.6907000542 \quad 5.6684999466-1.5128999949$

$\mathrm{H}-1.3767000437 \quad 3.5269999504 \quad 2.3594000340$

H $-2.5759000778 \quad 5.6627998352 \quad 1.7211999893$ 
$\mathrm{H}-3.9374001026 \quad 4.7076001167 \quad 1.1456999779$

$\mathrm{H}-2.8559999466 \quad 4.7651000023 \quad 4.0100998878$

$\mathrm{H}-4.3548998833 \quad 5.5047001839 \quad 3.4523000717$

$\mathrm{H}-4.7676000595 \quad 3.2925999165 \quad 4.5882000923$

H $-5.3034000397 \quad 3.2802000046 \quad 2.9105999470$

$\mathrm{H}-2.6705000401 \quad 2.1022999287 \quad 3.9660000801$

$\mathrm{H}-4.0622000694 \quad 1.1880999804 \quad 3.3903000355$

$\mathrm{H}-3.7555999756 \quad 2.0961000919 \quad 1.1060999632$

$\mathrm{H}-2.2565000057 \quad 1.3380000591 \quad 1.6643999815$

$\mathrm{H}-1.1492999792 \quad 5.9538998604 \quad 0.12129999697$

H $0.057999998331 \quad 5.2214999199 \quad 2.1830999851$

H $1.3848999739 \quad 4.6234998703 \quad 1.2167999744$

H $0.46079999208 \quad 7.5440998077 \quad 1.4131000042$

H $1.9240000248 \quad 6.8653998375 \quad 2.1194000244$

H $2.40709996228 .0960998535-0.028400000185$

H $2.8403999805 \quad 6.3926000595-0.14169999957$

H $0.27039998770 \quad 7.6707000732-1.2207000256$

H $1.6126999855 \quad 7.0739998817-2.1916000843$

H $1.1871999502 \quad 4.7473001480-1.4505000114$

H $-0.25929999352 \quad 5.4402999878 \quad-2.1449999809$

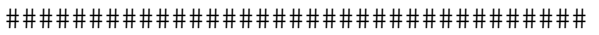

methylidene_ethylene

64

C $-0.035999998450 \quad 0.19529999793 \quad 0.12800000608$

C $2.8266000748 \quad 0.27500000596-0.014499999583$

$\mathrm{Ru} 1.85969996451 .8236000538 \quad 0.094599999487$

$\begin{array}{lllll}\text { Cl } & 1.3473000526 & 2.3110001087 & -2.2030999660\end{array}$

Cl $1.6869000196 \quad 2.1071999073 \quad 2.4781000614$

C $-0.57539999485 \quad 1.4316999912 \quad 0.26469999552$

$\mathrm{H}-0.76679998636 \quad 1.84930002691 .2476999760$

$\mathrm{H}-0.912299990651 .9886000156-0.60390001535$

H $0.21570000052-0.400700002910 .99919998646$

$\mathrm{H} \quad 0.072800002992-0.25900000334-0.85149997473$

H $2.3461999893-0.70819997787-0.010999999940$

H $3.9149000645 \quad 0.23919999599-0.079400002956$

P $3.78080010413 .2460999489-0.021500000730$

C $4.9197998047 \quad 3.1164000034 \quad 1.4821000099$

C $5.82030010221 .8608000278 \quad 1.4546999931$

C $6.5577998161 \quad 1.6723999977 \quad 2.7925999165$

C $7.3807001114 \quad 2.9133999348 \quad 3.1642000675$

C $6.4972000122 \quad 4.1683001518 \quad 3.1775999069$

C $5.7596001625 \quad 4.3656997681 \quad 1.8396999836$

C $4.75470018393 .1568999290-1.6286000013$

C $5.9788999557 \quad 4.0963997841-1.7024999857$

C $6.5748000145 \quad 4.1202998161-3.1217999458$

C $6.9432997704 \quad 2.7077999115-3.5984001160$

C $5.73409986501 .7653000355-3.5106000900$

C $5.12569999691 .7328000069-2.0962998867$

C 3.21160006525 .04930019380 .017899999395

C $2.5322999954 \quad 5.5501999855-1.2762000561$

C $2.2053999901 \quad 7.0520000458-1.1713000536$

C $1.33550000197 .3671998978 \quad 0.054000001401$

C $1.9889999628 \quad 6.8432002068 \quad 1.3408000469$

C $2.30979990965 .3421001434 \quad 1.2383999825$

H $4.1856999397 \quad 2.9658999443 \quad 2.2848000526$

H $5.2251000404 \quad 0.96740001440 \quad 1.2479000092$

H 6.56120014191 .95039999490 .64920002222

H $5.8186998367 \quad 1.4738999605 \quad 3.5806999207$

H $7.20289993290 .78710001707 \quad 2.7344000340$

H $7.8621001244 \quad 2.77530002594 .1398000717$

H $8.1892004013 \quad 3.04649996762 .4305000305$

H $5.7557001114 \quad 4.0813999176 \quad 3.9839999676$

H $7.0981001854 \quad 5.0592999458 \quad 3.3977999687$

H $6.4948000908 \quad 4.5690999031 \quad 1.0515999794$

H 5.12580013285 .25330019001 .9184000492

H $4.0019998550 \quad 3.5113000870-2.3445999622$

H $5.7119002342 \quad 5.1146998405-1.3992999792$

H $6.7498998642 \quad 3.7500998974-1.0024000406$

H $5.84040021904 .5584998131-3.8125000000$

H $7.4545998573 \quad 4.7753000259-3.1412999630$ 
H $7.32840013502 .7409000397-4.6248002052$

H $7.75680017472 .3162999153-2.9700999260$

H $4.96180009842 .0969998837-4.2185001373$

H $6.0181999207 \quad 0.74930000305-3.8106999397$

H $5.84689998631 .2774000168-1.4048999548$

H $4.22989988331 .1072000265-2.1050999165$

H $4.1375999451 \quad 5.6268000603 \quad 0.13869999349$

H $3.1782000065 \quad 5.3892998695-2.1449000835$

H $1.6181999445 \quad 4.9770002365-1.4643000364$

H $3.1438000202 \quad 7.6223001480-1.1050000191$

H $1.7056000233 \quad 7.3797001839-2.0910000801$

H $1.1563999653 \quad 8.4469003677 \quad 0.12739999592$

H $0.352100014696 .8916001320-0.069700002670$

H 2.91820001607 .40059995651 .5312999487

H $1.3358000517 \quad 7.01999998092 .2037999630$

H $1.3743000031 \quad 4.77710008621 .1416000128$

H $2.7699000835 \quad 4.99060010912 .1665999889$

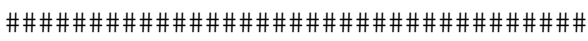

methylidene_pentene

73

C $-0.079599998891 \quad 0.47040000558 \quad 0.019400000572$

C $2.94490003590 .38429999352-0.095899999142$

$\begin{array}{lllll}\mathrm{Ru} & 0.87220001221 & 2.0213999748 & -0.072999998927\end{array}$

Cl $1.1447000504 \quad 2.2413001060-2.4574999809$

Cl $1.2611000538 \quad 2.5583999157 \quad 2.2399001122$

C $3.32599997521 .6864999533-0.12179999799$

C $2.9992001057-0.479699999091 .1339999437$

C $4.3031001091-1.30560004711 .1892999411$

C $4.3569998741-2.22480010992 .4133000374$

H $0.42689999938-0.488999992610 .16110000014$

$\mathrm{H}-1.1655000448 \quad 0.39469999075-0.056200001389$

H $2.7179000378-0.10289999843-1.0436999798$

H $3.4535000324 \quad 2.2097001076-1.0636999607$

H $3.6329998970 \quad 2.1914999485 \quad 0.78979998827$

H $2.9137001038 \quad 0.14669999480 \quad 2.0281999111$

H $2.1535000801-1.1808999777 \quad 1.1462999582$

H $4.3980998993-1.90209996700 .27200001478$

H $5.1572999954-0.61720001698 \quad 1.1994999647$

H $5.2915000916-2.79460000992 .4419000149$

H $4.2869000435-1.64769995213 .3420999050$

H $3.5281000137-2.94210004812 .4061000347$

P $-1.0424000025 \quad 3.4247000217-0.089699998498$

C $-2.1087999344 \quad 3.2685999870-1.6418999434$

C $-2.9730999470 \quad 1.9883999825-1.6655999422$

C $-3.63820004461 .7903000116-3.0392999649$

C $-4.4762001038 \quad 3.0104000568-3.4440999031$

C $-3.6307001114 \quad 4.2905001640-3.4035999775$

C $-2.9670000076 \quad 4.4962000847-2.0288999081$

C $-2.0829999447 \quad 3.3668000698 \quad 1.4756000042$

C $-3.2955000401 \quad 4.32280015951 .4972000122$

C $-3.9365999699 \quad 4.36789989472 .8959000111$

C $-4.3435997963 \quad 2.96580004693 .3710000515$

C $-3.1494998932 \quad 2.00130009653 .3285000324$

C $-2.4944999218 \quad 1.9517999887 \quad 1.9362000227$

C $-0.46900001168 \quad 5.2246999741-0.14749999344$

C 0.201800003655 .73600006101 .1468000412

C $0.53189998865 \quad 7.2360000610 \quad 1.0313999653$

C $1.41369998467 .5362000465-0.18880000710$

C $0.768999993807 .0036001205-1.4761999846$

C $0.44209998846 \quad 5.5044999123-1.3645999432$

H $-1.3345999718 \quad 3.1442999840 \quad-2.4105000496$

$\mathrm{H}-2.36269998551 .1109000444-1.4379999638$

$\mathrm{H}-3.75410008432 .0501999855-0.89600002766$

$\mathrm{H}-2.85520005231 .6183999777-3.7904999256$

$\mathrm{H}-4.26000022890 .88669997454 \quad-3.0188000202$

$\mathrm{H}-4.90299987792 .8666000366-4.4439997673$

$\mathrm{H}-5.3248000145 \quad 3.1142001152 \quad-2.7518000603$

H $-2.8475999832 \quad 4.2329998016-4.1726999283$

$\mathrm{H}-4.2459998131 \quad 5.1656999588-3.6461000443$

$\mathrm{H}-3.7469999790 \quad 4.6666998863 \quad-1.2771999836$ 
$\mathrm{H}-2.3587999344 \quad 5.4042000771 \quad-2.0657999516$

$\mathrm{H}-1.3532999754 \quad 3.7169001102 \quad 2.2172000408$

$\mathrm{H}-3.0055999756 \quad 5.3347001076 \quad 1.1936000586$

$\mathrm{H}-4.04720020293 .9788000584 \quad 0.77509999275$

$\mathrm{H}-3.2174000740 \quad 4.7994999886 \quad 3.6064000130$

$\mathrm{H}-4.8056001663 \quad 5.0373997688 \quad 2.8817999363$

H $-4.7602000237 \quad 3.0134000778 \quad 4.3843998909$

$\mathrm{H}-5.1437001228 \quad 2.58340001112 .7200999260$

$\mathrm{H}-2.39540004732 .3218998909 \quad 4.0605998039$

$\mathrm{H}-3.4625000954 \quad 0.99199998379 \quad 3.6226999760$

$\mathrm{H}-3.2000999451 \quad 1.5069999695 \quad 1.2216999531$

$\mathrm{H}-1.61199998861 .30850005151 .9766000509$

$\mathrm{H}-1.3933999538 \quad 5.8025999069-0.28119999170$

$\mathrm{H}-0.44810000062 \quad 5.5791997910 \quad 2.0132000446$

H 1.11459994325 .16540002821 .3449000120

H $-0.40439999104 \quad 7.80810022350 .95109999180$

$\mathrm{H} 1.0246000290 \quad 7.5711998940 \quad 1.9522000551$

H $1.59949994098 .6141004562-0.27050000429$

H 2.3933999538 $7.0566000938-0.052400000393$

$\mathrm{H}-0.15649999678 \quad 7.5629000664-1.6789000034$

$\mathrm{H} 1.42980003367 .1708002090-2.3354001045$

$\mathrm{H} 1.3738000393 \quad 4.9361000061-1.2596000433$

$\mathrm{H}-0.014700000174 \quad 5.1494998932-2.2932000160$

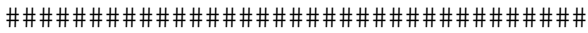

methylidene_zoctene

82

C $-0.12549999356 \quad 0.37560001016 \quad 0.0083999997005$

C $2.7960000038 \quad 0.32429999113 \quad 0.10069999844$

$\mathrm{Ru} 0.78839999437 \quad 1.9447000027 \quad 0.033100001514$

Cl $0.894100010402 .0074000359-2.4207000732$

$\begin{array}{lllll}\text { Cl } & 0.86320000887 & 1.9120999575 & 2.4881999493\end{array}$

C $3.17339992521 .6397999525 \quad 0.20649999380$

C $3.0000998974-0.55529999733-1.1055999994$

C $2.3492000103-1.9386999607-0.97790002823$

C $2.5401000977-2.7948999405-2.2332000732$

C $3.92980003362 .4230000973-0.84020000696$

C $5.43750000002 .4798998833-0.50940001011$

C $6.2258000374 \quad 3.2878999710-1.5448999405$

$\mathrm{H}-0.39730000496-0.13809999824 \quad 0.93790000677$

$\mathrm{H}-0.38240000606-0.11819999665-0.93489998579$

H $2.4477999210-0.153699994091 .0145000219$

H $3.1387999058 \quad 2.0720000267 \quad 1.2065000534$

H $2.6305999756-0.041600000113-2.0006999969$

H $4.0844001770-0.68569999933-1.2495000362$

H $2.7660000324-2.4593999386-0.10530000180$

н $1.2768000364-1.8207999468-0.77189999819$

H $2.0717000961-3.7776999474-2.1199998856$

H $2.0957999229-2.3087999821-3.1087999344$

H $3.6033999920-2.9542999268-2.4467999935$

H $3.7697999477 \quad 1.9995000362-1.8338999748$

H $3.54629993443 .4521999359-0.88179999590$

H $5.5763001442 \quad 2.9163000584 \quad 0.48870000243$

H $5.83239984511 .4571000338-0.45500001311$

H $7.29190015793 .3210000992-1.2968000174$

H $6.1266999245 \quad 2.8503999710-2.5446000099$

H $5.8619999886 \quad 4.3203001022-1.5993000269$

P $-1.0548000336 \quad 3.46639990810 .0066999997944$

C $-2.1059000492 \quad 3.4340999126-1.5422999859$

C $-2.6963999271 \quad 2.0497000217-1.8811000586$

C $-3.34439992902 .0715000629-3.2769000530$

C $-4.41389989853 .1684000492-3.3945999146$

C $-3.8427000046 \quad 4.5439000130-3.0192999840$

C $-3.1961998940 \quad 4.5244998932-1.6215000153$

C $-2.09879994393 .4040999413 \quad 1.5597000122$

C $-2.9191999435 \quad 4.6680998802 \quad 1.8995000124$

C $-3.5487999916 \quad 4.5359997749 \quad 3.2994000912$

C $-4.4123997688 \quad 3.2713999748 \quad 3.4193999767$

C $-3.6156001091 \quad 2.0144999027 \quad 3.0364999771$

C $-2.9925000668 \quad 2.14890003201 .6360000372$

C -0.305799990895 .19210004810 .023900000378$ 
C $0.61820000410 \quad 5.3935999870 \quad 1.2469999790$

C $1.1670999527 \quad 6.8295001984 \quad 1.2968000174$

C $1.8984999657 \quad 7.1989998817-0.0020999999251$

C $0.99229997396 \quad 6.9874000549-1.2235000134$

C $0.435900002725 .5531997681-1.2838000059$

$\mathrm{H}-1.3506000042 \quad 3.6307001114 \quad-2.3129999638$

$\mathrm{H}-1.9046000242 \quad 1.2998000383-1.8586000204$

$\mathrm{H}-3.45110011101 .7656999826-1.1368000507$

H $-2.5581998825 \quad 2.2411000729-4.0248999596$

$\mathrm{H}-3.7808001041 \quad 1.0894000530-3.4962999821$

$\mathrm{H}-4.8262000084 \quad 3.1939001083-4.4106001854$

H $-5.25159978872 .9321000576-2.7221000195$

$\mathrm{H}-3.0854001045 \quad 4.8387999535-3.7592999935$

$\mathrm{H}-4.6291999817 \quad 5.3083000183-3.0515000820$

$\mathrm{H}-3.9742000103 \quad 4.3225998878-0.87389999628$

$\mathrm{H}-2.7864000797 \quad 5.5148000717-1.3916000128$

$\mathrm{H}-1.3223999739 \quad 3.2818999290 \quad 2.3261001110$

H $-2.2918999195 \quad 5.56510019301 .8715000153$

$\mathrm{H}-3.7151000500 \quad 4.8154001236 \quad 1.1590000391$

$\mathrm{H}-2.7453999519 \quad 4.4995999336 \quad 4.0483999252$

$\mathrm{H}-4.1449999809 \quad 5.4300999641 \quad 3.5201001167$

$\mathrm{H}-4.8074002266 \quad 3.1777999401 \quad 4.4381999969$

$\mathrm{H}-5.2824997902 \quad 3.36509990692 .7528998852$

$\mathrm{H}-2.81150007251 .84979999073 .7662000656$

$\mathrm{H}-4.2613000870 \quad 1.1284999847 \quad 3.0706000328$

$\mathrm{H}-3.7967000008 \quad 2.21930003170 .89170002937$

H $-2.4094998837 \quad 1.2531000376 \quad 1.4009000063$

H $-1.1557999849 \quad 5.8814001083 \quad 0.12399999797$

H $0.094099998474 \quad 5.1567001343 \quad 2.1784000397$

$\mathrm{H} \quad 1.4551999569 \quad 4.6845002174 \quad 1.1944999695$

H $0.33410000801 \quad 7.5297999382 \quad 1.4566999674$

H $1.8358999491 \quad 6.9362998009 \quad 2.1593000889$

H 2.24909996998 .23750019070 .037700001150

H $2.7939999104 \quad 6.5687999725-0.10320000350$

H $0.154100000867 .6984000206-1.1797000170$

H $1.5382000208 \quad 7.2049999237-2.1494998932$

H $1.2524000406 \quad 4.8414998055-1.4566999674$

$\mathrm{H}-0.22939999402 \quad 5.4601001740-2.1473000050$

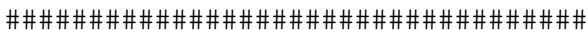

pcy3

52

P $0.0476000010972 .1212999821-0.23890000582$

C $1.88199996951 .6650999784-0.14810000360$

$\begin{array}{lllll}\text { C } 2.0687000751 & 0.15819999576 & -0.43799999356\end{array}$

C $3.5197999477-0.29370000958-0.19290000200$

C $4.5226001740 \quad 0.55119997263-0.99449998140$

C $4.3393998146 \quad 2.0502998829-0.71139997244$

C $2.88750004772 .4985001087-0.96749997139$

C $0.046500001103 \quad 3.9403998852 \quad 0.27540001273$

C -1.40880000594 .45979976650 .33590000868$

C -1.48300004015 .92500019070 .80099999905$

C $-0.79269999266 \quad 6.1181998253 \quad 2.1594998837$

C $0.65289998055 \quad 5.6002998352 \quad 2.1212000847$

C $0.72759997845 \quad 4.1368999481 \quad 1.6475000381$

C $-0.27739998698 \quad 2.1691000462-2.1143999100$

C $-1.7232999802 \quad 1.7059999704 \quad-2.4156999588$

C $-1.97660005091 .5571000576-3.9263000488$

C $-1.64240002632 .8480000496-4.6898999214$

C $-0.20829999447 \quad 3.3120999336-4.3927001953$

C $0.0350000001493 .4721000195-2.8794000149$

H 2.11800003051 .80850005150 .91670000553

$\mathrm{H} 1.3787000179-0.429800003770 .17820000648$

H $1.8066999912-0.050900001079-1.4848999977$

H $3.7460999489-0.201000005010 .87860000134$

H $3.6291999817-1.3559000492-0.44380000234$

H $5.5500001907 \quad 0.24140000343-0.76819998026$

H $4.3702998161 \quad 0.36860001087-2.0678000450$

H $4.5992999077 \quad 2.2550001144 \quad 0.33680000901$

H $5.0296998024 \quad 2.6424000263-1.3250000477$

H $2.66820001602 .3815000057-2.0367000103$ 
H $2.78169989593 .5657000542-0.74140000343$

$\mathrm{H} 0.59560000896 \quad 4.5401000977-0.46259999275$

H $-1.8950999975 \quad 4.3578000069-0.64069998264$

$\mathrm{H}-1.9794000387 \quad 3.8282001019 \quad 1.0304000378$

$\mathrm{H}-0.99570000172 \quad 6.5668997765 \quad 0.053399998695$

$\mathrm{H}-2.5299999714 \quad 6.2480001450 \quad 0.85390001535$

$\mathrm{H}-0.81279999018 \quad 7.1743001938 \quad 2.4544000626$

$\mathrm{H}-1.3523999453 \quad 5.5668997765 \quad 2.9281001091$

H $1.2416000366 \quad 6.2266001701 \quad 1.4361000061$

H $1.1205999851 \quad 5.6951999664 \quad 3.1087999344$

H $0.23880000412 \quad 3.4890999794 \quad 2.3891000748$

H $1.7767000198 \quad 3.8252999783 \quad 1.6047999859$

H $0.39199998975 \quad 1.3904000521-2.5093998909$

H $-1.9258999825 \quad 0.76010000706-1.9014999866$

$\mathrm{H}-2.4331998825 \quad 2.4358999729-2.0020999908$

$\mathrm{H}-1.3521000147 \quad 0.73930001259-4.3134999275$

$\mathrm{H}-3.0185000896 \quad 1.2649999857-4.1064000130$

$\mathrm{H}-1.78190004832 .7026998997-5.7680001259$

$\mathrm{H}-2.3443999290 \quad 3.6380000114 \quad-4.3873000145$

H $0.500999987132 .5748000145 \quad-4.7948999405$

H $0.00150000001304 .2583999634-4.9068999290$

$\mathrm{H}-0.60790002346 \quad 4.2793998718-2.5055999756$

H $1.0665999651 \quad 3.7906999588-2.7012999058$

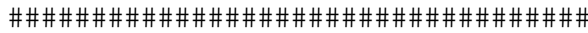

pentene

15

C $0.023900000378 \quad 1.5713000298 \quad-0.026200000197$

C $0.0031999999192 \quad 0.039799999446 \quad 0.0063999998383$

C $-1.4248000383-0.53949999809 \quad 0.021800000221$

C $-1.4490000010-2.0432000160 \quad 0.032200001180$

C $-1.9854999781-2.7962000370 \quad 0.99290001392$

$\mathrm{H} 1.0491000414 \quad 1.9564000368-0.038699999452$

$\mathrm{H}-0.48800000548 \quad 1.9566999674-0.91600000858$

$\mathrm{H}-0.47960001230 \quad 1.9926999807 \quad 0.85189998150$

H $0.54320001602-0.35519999266-0.86500000954$

$\mathrm{H} 0.54350000620-0.322200000290 .89029997587$

$\mathrm{H}-1.9692000151-0.155300006270 .89469999075$

$\mathrm{H}-1.9593000412-0.17049999535-0.86729997396$

$\mathrm{H}-0.97159999609-2.5327000618-0.81900000572$

$\mathrm{H}-1.9630999565-3.88179993630 .95120000839$

H $-2.4695000648-2.35060000421 .8601000309$

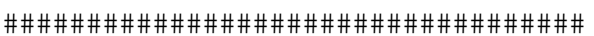

zoctene

24

C $0.34130001068 \quad 1.5247999430-0.44190001488$

C $0.21330000460 \quad 0.013500000350 \quad-0.23860000074$

C $-1.2470999956-0.45120000839-0.087600000203$

C $-1.3745000362-1.9428999424 \quad 0.067100003362$

C $-1.9268000126-2.6280000210 \quad 1.0770000219$

C $-2.5889999866-2.0866000652 \quad 2.3153998852$

$\begin{array}{lllll}\text { C }-4.0473999977 & -2.5574998856 & 2.4691998959\end{array}$

C $-4.7094998360-2.0448999405 \quad 3.7497000694$

H $1.3876999617 \quad 1.8262000084-0.55599999428$

$\mathrm{H}-0.199000000951 .8533999920-1.3375999928$

$\mathrm{H}-0.071699999273 \quad 2.0768001080 \quad 0.41060000658$

H $0.67299997807-0.51480001211-1.0852999687$

H $0.77969998121 \quad-0.29219999909 \quad 0.65069997311$

$\mathrm{H}-1.70700001720 .0767000019550 .75629997253$

$\mathrm{H}-1.8056000471-0.13339999318-0.98220002651$

$\mathrm{H}-0.93870002031-2.5188999176-0.75160002708$

$\mathrm{H}-1.9197000265-3.7172000408 \quad 1.0037000179$

$\mathrm{H}-2.0211999416-2.41680002213 .1995999813$

$\mathrm{H}-2.5604000092 \quad-0.99070000648 \quad 2.3308999538$

$\mathrm{H}-4.6216998100 \quad-2.2281999588 \quad 1.5936000347$

$\mathrm{H}-4.0735998154-3.65580010412 .4519999027$

$\mathrm{H}-5.7413001060-2.4007999897 \quad 3.8376998901$

$\mathrm{H}-4.1648998260-2.3798000813 \quad 4.6403999329$

$\mathrm{H}-4.7335000038-0.949000000953 .7732000351$

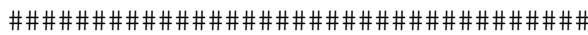


DOW RESTRICTED - For internal use only 